\title{
S2k-Leitlinie Gastrointestinale Blutung
}

AWMF-Register Nr. 021-28

\section{S2k Guideline Gastrointestinal Bleeding}

\section{Guideline of the German Society of Gastroenterology DGVS}

Authors

Martin Götz ${ }^{1}$, Mario Anders², Erwin Biecker ${ }^{3}$, Christian Bojarski ${ }^{4}$, Georg Braun ${ }^{5}$, Thorsten Brechmann ${ }^{6}$, Alexander Dechêne ${ }^{7}$, Matthias Dollinger ${ }^{8}$, Meinrad Gawaz ${ }^{9}$, Ralf Kiesslich ${ }^{10}$, Dieter Schilling ${ }^{11}$, Frank Tacke ${ }^{12}$, Alexander Zipprich ${ }^{13}$, Jonel Trebicka ${ }^{14,} 15$

Collaborators:

Tilo Andus, Beate Appenrodt, Andrik Aschoff, Daniel Benten, Karel Caca, Ulrike Denzer, Helmut Diepolder, Wolfgang Fischbach, Bernhard Gebauer, Alexander L. Gerbes, Veit Gülberg, Horst Hohn, Ralf Jakobs, Markus Juchems, Michael Jung, Martin Keuchel, Robert Klamroth, Thomas Leyhe, Petra Lynen-Jansen, Alexander Meining,

Helmut Messmann, Ralf Metzger, Jonas Mudter, Horst Neuhaus, Johannes W. Rey, Andrea Riphaus, Elke Roeb, Fred Salomon, Anja Schaible, Michael Schultheiß, Dirk Sibbing, Alfred Simon, Christian P. Strassburg Fachgesellschaften:

Deutsche Gesellschaft für Gastroenterologie, Verdauungs- und Stoffwechselkrankheiten (DGVS) (federführend), Deutschen Morbus Crohn und Colitis ulcerosa Vereinigung (DCCV), Deutsche Röntgengesellschaft (DRG), Deutsche Gesellschaft für interventionelle Radiologie (DeGiR), Deutsche Gesellschaft für Allgemein- und Viszeralchirurgie (DGAV) und Chirurgische Arbeitsgemeinschaft für Endoskopie und Sonographie (CAES) der DGAV, Deutsche Gesellschaft für Internistische Intensivmedizin (DGIIN), Deutsche Gesellschaft für Innere Medizin (DGIM), Deutsche Gesellschaft für Kardiologie (DGK), Akademie für Ethik in der Medizin (AEM), Gesellschaft für Thrombose- und Hämostaseforschung (GTH)

Institute

1 Innere Medizin I, Gastroenterologie, Hepatologie, Infektiologie, Universitätsklinikum Tübingen

2 Klinik für Innere Medizin, Vivantes Wenckebach-Klinikum

3 Abteilung Innere Medizin, Zollernalb Klinikum

4 Medizinische Klinik für Gastroenterologie, Infektiologie und Rheumatologie, Charité - Campus Benjamin Franklin

5 III. Medizinische Klinik, Klinikum Augsburg

6 Medizinische Klinik I, Klinikum der Ruhr-Universität Bochum, Berufsgen. Kliniken Bergmannsheil

7 Klinik für Gastroenterologie und Hepatologie, Universitätsklinikum Essen

8 Medizinische Klinik I, Klinikum Landshut

9 Innere Medizin III, Universitätsklinikum Tübingen

10 II. Medizinische Klinik, HSK Dr.-Horst-Schmidt-Kliniken

11 Medizinische Klinik II, Diakonissenkrankenhaus Mannheim

12 Medizinische Klinik III, Universitätsklinikum Aachen

13 Universitätsklinik und Poliklinik Innere Medizin I, Universitätsklinikum Halle
14 Medizinische Klinik und Poliklinik I, Universitätsklinikum Bonn

15 European Foundation for Study of Chronic Liver Failure, Barcelona, Spain

Bibliografie

DOI https://doi.org/10.1055/s-0043-116856

Z Gastroenterol 2017; 55: 883-936

(c) Georg Thieme Verlag KG Stuttgart · New York

ISSN 0044-2771

Korrespondenzadresse

Univ. Prof. Dr. med. Martin Götz

Innere Medizin I, Gastroenterologie, Hepatologie,

Infektiologie, Universitätsklinikum Tübingen,

Otfried-Müller-Str. 10, 72076 Tübingen

Tel.: ++49/7071/2968889

martin.goetz@med.uni-tuebingen.de 


\section{ANSPRECHPARTNER FÜR DIE AKTUALISIERUNG}

Priv.-Doz. Dr. med. Petra Lynen Jansen

Deutsche Gesellschaft für Gastroenterologie, Verdauungsund Stoffwechselkrankheiten

Olivaer Platz 7

10707 Berlin

Tel. 030/3198315003

lynen@dgvs.de

\section{Einleitung}

Gastrointestinale Blutungen sind häufig. Ihr klinisches Spektrum reicht von der nur laborchemisch fassbaren Anämie bis hin zur fulminanten Blutung mit Schock und erfordert ein differenziertes Vorgehen vom ersten Verdacht einer gastrointestinalen Blutung über die Akutversorgung bis hin zur Prävention einer erneuten Blutung.

Diese interdisziplinäre Leitlinie unter Leitung der DGVS und Beteiligung benachbarter Fachgesellschaften fasst die wichtigsten Aspekte zur Versorgung von Patienten mit gastrointestinaler Blutung unter Berücksichtigung der aktuellen Datenlage zusammen. Insbesondere wurden Praktikabilität und Einfachheit der Empfehlungen betont, um die Umsetzung im klinischen Alltag zu vereinfachen, wohlwissend, dass ein Querschnitt der Datenlage immer in eine Einzelfallentscheidung am individuellen Patienten überführt werden muss. Die Schnittmenge mit verschiedenen weiteren Leitlinien wurde bewusst herausgestellt und, soweit erforderlich, aktualisiert.

Wir hoffen, dass diese Leitlinie eine Stütze für die Versorgung der Patienten mit einer gastrointestinalen Blutung ist und auch die Aspekte betont, in denen weiterer Forschungsbedarf gesehen wird.

\section{Arbeitsgruppe 1: Prä-endoskopisches Management}

Die Empfehlungen zum prä-endoskopischen Management sind in der $\triangleright$ Abb. 1 zusammengefasst.

\section{Initiale Risikoeinschätzung}

\section{EMPFEHLUNG 1}

Zu einer ersten Risikoeinschätzung bei vermuteter gastrointestinaler Blutung sollten eine (Fremd-)Anamnese, der körperliche Untersuchungsbefund und die Erhebung der Vitalparameter herangezogen werden.

Empfehlung, starker Konsens.

Eine initiale Anamnese sollte u. a. Fragen nach der Blutungsmanifestation und -dauer (Erbrechen von Kaffeesatz, Hämatemesis,

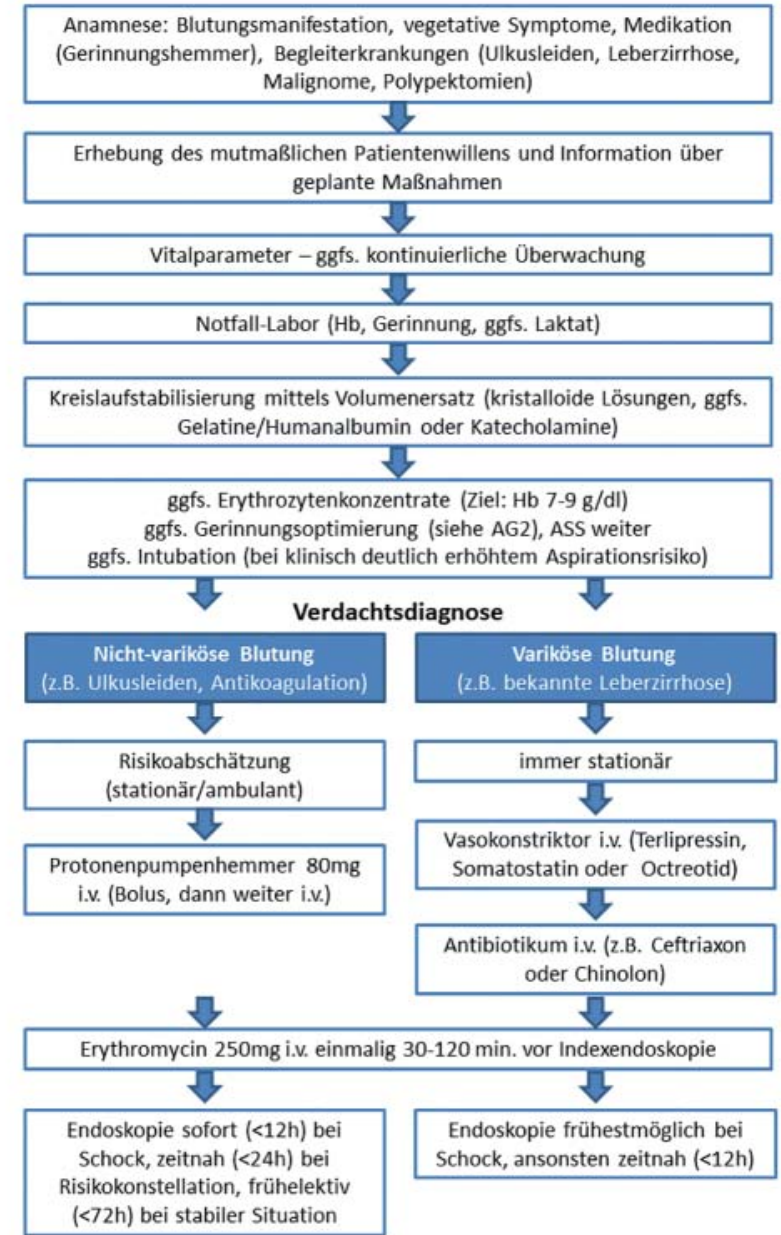

- Abb. 1 Präendoskopisches Management bei vermuteter gastrointestinaler Blutung. Einzelheiten ergeben sich aus den Leitlinienempfehlungen (siehe Text).

Teerstuhl, Hämatochezie bzw. Abgang von Blutkoageln), nach Begleitsymptomen (orthostatische Dysregulation, Synkope, Vigilanzminderung, Aspiration, Agitation), nach der Medikation (insbesondere bzgl. Thrombozytenaggregationshemmung mit Acetylsalicylsäure, ggf. als duale Thrombozytenaggregationshemmung [DAPT] in Kombination mit Clopidogrel, Prasugrel bzw. Ticagrelor, orale Antikoagulation mit Vitamin-K-Antagonisten oder mit dem Thrombininhibitor Dabigatran bzw. den FaktorXa-Inhibitoren Rivaroxaban, Apixaban oder Edoxaban als direkte orale Antikoagulanzien [DOAK], Antikoagulation mit unfraktioniertem Heparin intravenös bzw. niedermolekularen Heparinen subkutan, nicht-steroidale Antiphlogistika [NSAR]) und nach Begleit- bzw. Vorerkrankungen (Leberzirrhose, splanchnische Thrombosen, Ulkusleiden, stattgehabte variköse oder nicht-variköse gastrointestinale Blutungen, Malignome des Gastrointestinaltrakts, hämatologische Neoplasien, kürzlich stattgehabte PT(C)A, kürzlich stattgehabte Polypektomie) beinhalten ( $\triangleright$ Tab. 1) [1, 2]. Eine digital-rektale Untersuchung inklusive Test auf okkultes Blut sollte im Rahmen der körperlichen Untersuchung erfolgen. Eine nicht-invasive Blutdruckmessung sowie eine 
- Tab. 1 Wichtige Aspekte in der Anamnese zur Risikoabschätzung bei Verdacht auf gastrointestinale Blutung.

\section{Anamnese zur Risikoabschätzung bei vermuteter gastrointestinaler Blutung}

\begin{tabular}{|c|c|}
\hline \multirow{5}{*}{$\begin{array}{l}\text { Blutungs- } \\
\text { manifestation }\end{array}$} & Erbrechen von Kaffeesatz \\
\hline & Hämatemesis \\
\hline & Teerstuhl \\
\hline & Hämatochezie \\
\hline & peranaler Abgang von Blutkoageln \\
\hline \multirow[t]{5}{*}{ Begleitsymptome } & orthostatische Dysregulation \\
\hline & Synkope \\
\hline & Vigilanzminderung \\
\hline & stattgehabte Aspiration \\
\hline & Agitation \\
\hline \multirow[t]{7}{*}{ Medikation } & Thrombozytenaggregationshemmung \\
\hline & duale Thrombozytenaggregationshemmung \\
\hline & Vitamin-K-Antagonisten \\
\hline & „Triple-Therapie“ \\
\hline & direkte orale Antikoagulanzien \\
\hline & Heparin \\
\hline & nicht-steroidale Antirheumatika \\
\hline \multirow[t]{7}{*}{ Vorerkrankungen } & Leberzirrhose, splanchnische Thrombosen \\
\hline & Ulkusleiden \\
\hline & $\begin{array}{l}\text { stattgehabte variköse oder nicht-variköse } \\
\text { gastrointestinale Blutungen }\end{array}$ \\
\hline & Malignome des Gastrointestinaltrakts \\
\hline & Hämatologische Neoplasien \\
\hline & kürzlich stattgehabte PT(C)A \\
\hline & $\begin{array}{l}\text { kürzlich stattgehabte Polypektomie oder andere } \\
\text { Eingriffe im GI-Trakt }\end{array}$ \\
\hline
\end{tabular}

Bestimmung der Herzfrequenz und der peripheren Sauerstoffsättigung $\mathrm{SpO}_{2}$ sollten erfolgen [2].

\section{EMPFEHLUNG 2}

Bei Patienten mit vermuteter gastrointestinaler Blutung soll der Hämoglobinwert bestimmt werden.

Starke Empfehlung, starker Konsens.

\section{EMPFEHLUNG 3}

Eine Point-of-care-Diagnostik inklusive Laktatbestimmung kann zur Risikostratifizierung bei Patienten mit vermuteter gastrointestinaler Blutung herangezogen werden.

Offene Empfehlung, starker Konsens.
Der Schweregrad einer gastrointestinalen Blutung kann anhand des Hämoglobin-Werts abgeschätzt werden, der z. B. im Rahmen einer Blutgasanalyse (Point-of-care-Diagnostik) unmittelbar verfügbar ist [1]. Der Hämoglobinwert ist auch im ambulanten Umfeld schnell verfügbar und unterstützt die klinische Einschätzung des Ausmaßes einer vermuteten gastrointestinalen Blutung. Je nach klinischer Situation (z. B. vermutete chronische Blutung, vermutete Varizenblutung, vermutete Hämorrhoidenblutung) muss der Hämoglobinwert aber nicht vor Einleiten weiterer Maßnahmen abgewartet werden.

Im septischen Schock ist eine Laktaterhöhung als prognostischer Parameter unstrittig [3], besonders die Laktat-Clearance innerhalb der ersten Stunden scheint für das Outcome von Bedeutung [4]. Kürzlich konnte gezeigt werden, dass auch bei der oberen gastrointestinalen Blutung ein signifikanter Zusammenhang zwischen dem Ausmaß der Laktaterhöhung innerhalb der ersten $24 \mathrm{~h}$ und der Sterblichkeit auf der Intensivstation besteht [5]. Insbesondere erhöhte Laktatwerte ( $>5 \mathrm{mmol} / \mathrm{l}$ ) bei Aufnahme im Krankenhaus zeigen daher möglicherweise ein erhöhtes Letalitätsrisiko an und könnten zur Risikostratifizierung herangezogen werden [5]. Zur Bewertung der Gerinnungssituation wird auf die Empfehlungen der AG2 verwiesen.

\section{AUSSAGE 1 \\ Es ist nicht möglich, aufgrund nicht-invasiver Marker sicher zwischen nicht-varikösen und varikösen Blutungsquellen bei oberer gastrointestinaler Blutung zu unterscheiden, aber Risi- kofaktoren wie Leberzirrhose, splanchnische Thrombosen, Thrombozytopenie oder erhöhte Leber-/Milz-Gewebesteifig- keit können Hinweis auf eine variköse Blutungsursache im oberen Gastrointestinaltrakt sein. \\ Offene Empfehlung, starker Konsens.}

Es ist sehr schwierig, bei Patienten mit oberer gastrointestinaler Blutung aufgrund der Anamnese und anderer nicht-invasiver Indikatoren zwischen variköser und nicht-variköser Blutungsquelle zu unterscheiden. In einer großen retrospektiven Analyse von 2233 stationär aufgenommenen Patienten mit oberer gastrointestinaler Blutung, davon 1034 Patienten mit Leberzirrhose, waren Thrombozytopenie, AST-Thrombozyten-Ratio (APRI-Score), ASTALT-Ratio (AAR) und der Lok-Index (nicht-invasive Labortests) hinweisend auf das Vorhandensein von Varizen sowie mit niedrigerer Sensitivität und Spezifität auch hinweisend auf eine Varizenblutung [6]. Leider war keiner der untersuchten Parameter in multivariaten Untersuchungen in der Lage, bei Patienten mit bekannter Leberzirrhose sicher zwischen nicht-varikösen und varikösen Blutungen zu unterscheiden [6]. Derzeit werden weitere Verfahren evaluiert; beispielsweise scheinen sowohl die Lebersteifigkeit, gemessen über transiente Elastografie (Fibroscan ${ }^{\circledR}$, Fa. Echosense) oder andere Ultraschall-basierte Methoden (z. B. Acoustic Radiation Force Impulse Imaging, ARFI; Scherwellenelastografie, SWE), und die Steifigkeit der Milz sowohl das Vorliegen von Varizen bei Leberzirrhose [7 - 9] als auch das Blutungsrisiko [10] vorherzusa- 
gen. Der Stellenwert in der akuten Blutungssituation ist aber aktuell noch nicht definiert.

\section{EMPFEHLUNG 4}

Zur präendoskopischen Risikostratifizierung bei vermuteter nicht-variköser oberer gastrointestinaler Blutung kann der (modifizierte) Glasgow-Blatchford-Score (GBS) bestimmt werden. Patienten mit einem (m)GBS von 0 - 1 können einer ambulanten Gastroskopie im Verlauf zugeführt werden.

Offene Empfehlung, starker Konsens.

Für die präendoskopische Risikobewertung sollte der Rockall-

Score nicht verwendet werden.

Empfehlung, starker Konsens.

Im Jahr 2000 wurde ein Scoring-Modell für die nicht-variköse obere gastrointestinale Blutung vorgeschlagen [11], in den sogenannten Glasgow-Blatchford-Score (GBS) fließen klinische Parameter, Vitalparameter, Laborwerte und Vorerkrankungen mit ein. Anhand des Scores gelang es auch in prospektiven Studien, mit hoher Sensitivität und Spezifität die Notwendigkeit einer endoskopischen Intervention vorauszusagen. Ein vereinfachter bzw. modifizierter Glasgow-Blatchford-Score (mGBS) basiert unter Ausschluss subjektiver Kriterien ausschließlich auf Vital- und Laborparametern mit ähnlich guter Sensitivität und Spezifität (ROC-AUC 0,85) ( Tab. 2) [12].

Patienten mit einer Niedrig-Risiko-Konstellation (0 - 1 Punkt) im Glasgow-Blatchford-Score (Hb $\left(o^{\top}\right) \geq 130 \mathrm{~g} / \mathrm{l} \mathrm{bzw}$. Hb (우) $\geq 120 \mathrm{~g} / \mathrm{l}$, systolischer Blutdruck $\geq 110 \mathrm{mmHg}$, Puls $<100 / \mathrm{min}$, Fehlen von Meläna, Synkope, Herz- oder Lebererkrankungen) benötigten mit einer Sensitivität >99\% keine endoskopische Intervention [13]. Eine ambulante Versorgung mittels Gastroskopie im Verlauf ist gerechtfertigt. Einschränkend anzumerken ist, dass der Glasgow-Blatchford-Score eine evtl. vorhandene antithrombotische Therapie nicht berücksichtigt, außerdem ist er nicht für intensivmedizinische oder postoperative Patienten validiert.

Der präendoskopische Rockall-Score [14] ist für die initiale Risikostratifizierung nicht geeignet. Er erlaubt keine zuverlässige Aussage über die Notwendigkeit der Durchführung einer endoskopischen Intervention $[13,15]$.

Daher können Scores die klinische Entscheidungsfindung erleichtern, diese aber nicht ersetzen.

\section{EMPFEHLUNG 5}

Patienten mit vermuteter variköser oberer gastrointestinaler Blutung sollen unter stationären Bedingungen evaluiert werden.

Starke Empfehlung, starker Konsens.
- Tab. 2 Modifizierter Glasgow-Blatchford-Score (mGBS) zur Risikoabschätzung bei vermuteter nicht-variköser oberer gastrointestinaler Blutung (adaptiert nach [12]).

\begin{tabular}{|c|c|c|}
\hline Kriterium & Ausprägung & Punktzahl \\
\hline Herzfrequenz & $\geq 100 / \mathrm{min}$ & 1 \\
\hline \multirow[t]{3}{*}{ systolischer RR } & $100-109 \mathrm{mmHg}$ & 1 \\
\hline & $90-99 \mathrm{mmHg}$ & 2 \\
\hline & $<90 \mathrm{mmHg}$ & 3 \\
\hline \multirow[t]{4}{*}{ Harnstoff (mg/dl) } & $\begin{array}{l}\geq 18,2 \text { und } \\
<22,4 \mathrm{mg} / \mathrm{dl}\end{array}$ & 2 \\
\hline & $\begin{array}{l}\geq 22,4 \text { und } \\
<28 \mathrm{mg} / \text { dl }\end{array}$ & 3 \\
\hline & $\geq 28$ und $<70 \mathrm{mg} / \mathrm{dl}$ & 4 \\
\hline & $\geq 70 \mathrm{mg} / \mathrm{dl}$ & 6 \\
\hline \multirow[t]{3}{*}{ Hämoglobin $\left(\sigma^{\pi}\right)$} & $\geq 12$ und $<13 \mathrm{~g} / \mathrm{dl}$ & 1 \\
\hline & $\geq 10$ und $<12 \mathrm{~g} / \mathrm{dl}$ & 3 \\
\hline & $<10 \mathrm{~g} / \mathrm{dl}$ & 6 \\
\hline \multirow[t]{2}{*}{ Hämoglobin (+) } & $\geq 10$ und $<12 \mathrm{~g} / \mathrm{dl}$ & 3 \\
\hline & $<10 \mathrm{~g} / \mathrm{dl}$ & 6 \\
\hline
\end{tabular}

Anmerkung : Harnstoff ursprünglich als Blood urea nitrogen (BUN) angegeben; Harnstoff: Umrechnung von mmol/l in mg/dl: Harnstoff in $\mathrm{mmol} / \mathrm{l} / 0,1665=$ Harnstoff in $\mathrm{mg} / \mathrm{dl}$; Umrechnung von Harnstoff in BUN: Harnstoff in mg/dl/2142 = BUN in mg/dl. Bewertung: Niedrigrisiko-Gruppe: 0 - 1 Punkt; Maximale Punktzahl: 16 Punkte.

Patienten mit vorbestehender Lebererkrankung und gastrointestinaler Blutung sind Hochrisikopatienten mit einer erheblichen Letalität, die einer stationären Überwachung und Therapie bedürfen [16].

\section{EMPFEHLUNG 6A}

Patienten mit vermuteter unterer gastrointestinaler Blutung (okkultes Blut im Stuhl, gelegentliche Blutauflagerungen) können bei eindeutig stabiler klinischer Situation und niedrigem Risikoprofil ambulant versorgt werden. Offene Empfehlung, starker Konsens.

\section{EMPFEHLUNG 6B}

Patienten mit vermuteter unterer gastrointestinaler Blutung und Hinweisen auf eine akute Gefährdung durch die Blutung (z. B. eine intermittierend schwere Hämatochezie mit vegetativer Reaktion) sollten stationär versorgt werden. Empfehlung, starker Konsens. 
Es gibt keine randomisierten prospektiven Studien zur optimalen Versorgung der „harmlosen“ unteren gastrointestinalen Blutung. Anders als bei der oberen gastrointestinalen Blutung gibt es für die unteren gastrointestinalen Blutungen auch keine allgemein akzeptierten oder multizentrisch prospektiv validierten Scores [17]. In vielen, zumeist retrospektiven Studien zeigte sich insgesamt eine sehr niedrige blutungsassoziierte Letalität selbst bei akuter unterer gastrointestinaler Blutung [5, 17]. Eine größere japanische Studie (439 Patienten) konnte acht Risikofaktoren für eine schwere akute gastrointestinale Blutung bei stationär aufgenommenen Patienten herausarbeiten, welche in einer kleineren prospektiven Kohorte (161 Patienten) validiert wurden: Einnahme von nicht-steroidalen Antiphlogistika (NSAR), Diarrhö, abdominelle Abwehrspannung, systolischer Blutdruck unter $100 \mathrm{mmHg}$, Thrombozytenaggregationshemmung, Albuminspiegel unter $3 \mathrm{~g} / \mathrm{dL}$, mehrere Begleiterkrankungen (Charlson Comorbidity Index $\geq 2$ ) und Synkope/Kollaps („NOBLADS Score“) [18]. Dieser Score war mit mehreren Endpunkten wie Transfusionsbedarf, Interventionen und Krankenhausaufenthaltsdauer assoziiert, umgekehrt benötigte nur ein Patient (von 66 Patienten) mit einem Score von 0 eine Transfusion und eine endoskopische Intervention [18]. In einer erst kürzlich publizierten Untersuchung [19] verglichen die Autoren die Genauigkeit dreier Scores (ASA, Center of Ulcer Research and Education Hemostatis Score und Charlson-Index), um einen schweren Verlauf der unteren gastrointestinalen Blutung vorherzusagen. Allerdings hatten alle Scores außer dem ASA-Score keine gute diagnostische Aussagefähigkeit (accuracy $75 \%$ maximal). Somit können Scores in der klinischen Routine auch nicht empfohlen werden. Davon ausgenommen bleibt der ASA-Score, der alleine schon zur Evaluation des Sedierungsrisikos bestimmt werden sollte. Dies deckt sich mit retrospektiven Analysen stationär versorgter Patienten, nach denen bei eindeutig stabiler klinischer Situation (Hämoglobin-Wert > $13 \mathrm{~g} / \mathrm{dl}$, systolischer Blutdruck > $115 \mathrm{mmHg}$ ) und Fehlen von Risikofaktoren (d. h. keine Antikoagulation, keine schweren Komorbiditäten) keine stationäre Aufnahme erforderlich ist $[20,21]$. Selbstverständlich ist okkultes oder overtes Blut im Stuhl aber immer als Warnsymptom aufzufassen, das eine zeitnahe (dann ggfs. ambulante) Abklärung mit einer Koloskopie nach sich ziehen sollte [2]. Damit kommt der klinischen Einschätzung (Symptomatik/Kreislaufreaktion, Komorbiditäten, Schwere der Blutung, Hb-Wert bei chronischem Blutverlust, Begleitmedikation/Antikoagulation, Versorgungssituation des Patienten, stattgehabte Interventionen wie Polypektomie) eine bestimmende Bedeutung für die Entscheidung zur ambulanten oder stationären Abklärung zu.

\section{Zeitpunkt der Endoskopie und Überwachung bis zur Endoskopie}

\section{EMPFEHLUNG 7}

Bei hämorrhagischem Schock bei vermuteter nicht-variköser oberer gastrointestinaler Blutung sollte unmittelbar nach Maßnahmen zur Kreislaufstabilisierung eine Endoskopie erfolgen (innerhalb von $12 \mathrm{~h}$ ). Bei Hochrisikosituationen und vermuteter nicht-variköser oberer gastrointestinaler Blutung sollte zeitnah (innerhalb von $24 \mathrm{~h}$ ) eine Endoskopie erfolgen. Bei vermuteter nicht-variköser oberer gastrointestinaler Blutung und hämodynamischer Stabilität sollte frühelektiv (innerhalb von $72 \mathrm{~h}$ ) eine Endoskopie erfolgen.

Offene Empfehlung, starker Konsens.

Zur Einschätzung der Dringlichkeit kann der Glasgow-Blatchford-Score eingesetzt werden.

Empfehlung, starker Konsens.

Bei hämorrhagischem Schock bei vermuteter nicht-variköser oberer gastrointestinaler Blutung sollte die Aufnahme auf eine Intensivstation erfolgen. Nach hämodynamischer Stabilisierung sollte zeitnah eine ÖGD erfolgen, in der ESGE-Leitlinie zur nicht-varikösen oberen gastrointestinalen Blutung ist hierfür ein Zeitfenster $<12 \mathrm{~h}$ vorgesehen [1].

Eine Endoskopie innerhalb der ersten zwölf Stunden nach Aufnahme resultierte in einer prospektiven, randomisierten Studie an 325 Patienten in einem signifikant geringeren Bedarf an Erythrozytenkonzentraten ( $450 \mathrm{ml}$ vs. $666 \mathrm{ml}, \mathrm{P}<0,001)$ [22].

Die ESGE-Leitlinie empfiehlt ebenfalls eine zeitnahe Endoskopie innerhalb der ersten zwölf Stunden bei beobachteter Hämatemesis und bei Patienten mit vermuteter oberer gastrointestinaler Blutung, bei denen die antithrombotische Therapie fortgesetzt werden muss [1].

Bei 97 Patienten mit einem Glasgow-Blatchford-Score GBS $\geq 12$ konnte in einer retrospektiven Analyse die Mortalität mittels Endoskopie mit erfolgreicher Blutstillung innerhalb der ersten 13 Stunden signifikant gesenkt werden ( 0 vs. $44 \%, P<0,001$ ), sodass eine ÖGD in einem Zeitfenster $<24 \mathrm{~h}$ erfolgen sollte [23].

Zur Identifikation von Hochrisikopatienten können neben klassischen anamnestischen Parametern (bekanntes Ulkusleiden, vorangegangene gastrointestinale Blutungen, gastrointestinales Tumorleiden, laufende Thrombozytenaggregationshemmung oder Antikoagulation, hohes Alter, schwere Begleiterkrankungen) auch einfache klinische Einschätzungen wie der „AIMS65-Score“ (Albumin, INR, mental status, systolic RR, age $>65$ ) herangezogen werden. Dieser beinhaltet niedriges Albumin $(<3 \mathrm{~g} / \mathrm{dl})$, hoher INR $(>1,5)$, Bewusstseinstrübung („altered mental status“), niedriger systolischer Blutdruck (<90 mmHg) und Alter (> 65 Jahre) [24].

Bei vermuteter oberer gastrointestinaler Blutung bei einem hämodynamisch stabilen Patienten sollte eine ÖGD innerhalb der ersten $72 \mathrm{~h}$ erfolgen [2].

\section{EMPFEHLUNG 8}

Bei vermuteter variköser oberer gastrointestinaler Blutung sollte eine kontinuierliche Überwachung der Vitalparameter sowie zeitnah $(<12 \mathrm{~h}$ ) eine Endoskopie erfolgen. Bei vermuteter variköser Blutung mit hämodynamischer Instabilität sollte frühestmöglich eine Notfall-Endoskopie erfolgen. Empfehlung, starker Konsens.

Bei vermuteter variköser oberer gastrointestinaler Blutung mit hämodynamischer Instabilität sollte die Aufnahme auf eine Intensiv- 
station erfolgen, bei hämodynamischer Stabilität sollte bis zur Endoskopie zumindest eine kontinuierliche Überwachung der Vitalparameter gewährleistet sein. Nach hämodynamischer Stabilisierung, intravenöser Applikation einer vasoaktiven Substanz und eines Breitspektrumantibiotikums und ggf. nach endotrachealer Intubation (siehe Empfehlungen unten) sollte zeitnah ( $<12 \mathrm{~h}$ ) eine Gastroskopie erfolgen [25 - 28]. Hämodynamisch instabile Patienten sollten frühestmöglich (d. h. sobald es der Allgemeinzustand des Patienten zulässt) einer Notfall-Endoskopie zugeführt werden [29]. Mittels Kombination aus medikamentöser Therapie und frühzeitiger, effektiver endoskopischer Intervention konnte die Mortalität der varikösen Blutung in den letzten Jahren kontinuierlich gesenkt werden [30].

\section{EMPFEHLUNG 9}

Bei hämorrhagischem Schock bei vermuteter unterer gastrointestinaler Blutung sollten nach Maßnahmen zur Kreislaufstabilisierung unmittelbar (innerhalb von $12 \mathrm{~h}$ ) endoskopische Untersuchungen (Gastroskopie plus ggfs. Rekto-Sigmoidooder Koloskopie) erfolgen. Bei hämodynamischer Stabilität und vermuteter unterer gastrointestinaler Blutung sollte eine Koloskopie erst nach entsprechender Vorbereitung erfolgen. Empfehlung, starker Konsens.

Schwere Verläufe bei der unteren gastrointestinalen Blutung sind seltener, die Mortalitätsrate geringer [31], dennoch kommt es besonders bei älteren Patienten > 65 Jahren [18] und unter antithrombotischer Therapie, insbesondere unter direkten oralen Antikoagulanzien [32, 33], häufiger zu schweren Verläufen. Als Risikofaktoren für einen schweren Verlauf einer unteren gastrointestinalen Blutung gelten eine Herzfrequenz > 100/min, ein systolischer Blutdruck $<115 \mathrm{mmHg}$, ein synkopales Ereignis, ein akutes Abdomen, peranaler Blutabgang bis vier Stunden nach Krankenhausaufnahme, ASS-Einnahme und mehr als zwei relevante Begleiterkrankungen. Sollten mehr als drei Risikofaktoren vorliegen, so besteht eine Mortalität von 9,6\% [34, 35].

Bei hämorrhagischem Schock bei vermuteter unterer gastrointestinaler Blutung sollte die Aufnahme auf eine Intensivstation erfolgen.

Bei hämorrhagischem Schock bei Hämatochezie und/oder peranalem Abgang von Blutkoageln sollte zunächst eine Gastroskopie erfolgen. Nach Ausschluss einer Blutungsquelle im oberen Gastrointestinaltrakt kann dann im Falle einer medikamentös nicht zu beherrschenden Kreislaufsituation eine Koloskopie auch bei unvorbereitetem Kolon erfolgen [36]. Immerhin gelang es in einer kleinen Fallserie $(n=13)$ bei bis zu $40 \%$ der Patienten, bei unvorbereitetem Kolon die Blutungsquelle zu lokalisieren, das Zökum wurde bei $69 \%$ der Fälle erreicht [37].

Bei hämodynamischer Stabilität sollte erst nach adäquater Vorbereitung (Darmreinigung) eine Koloskopie erfolgen [2].

\section{Strukturelle Anforderungen}

\section{EMPFEHLUNG 10A}

Patienten mit vermuteter gastrointestinaler Blutung sollen situationsadaptiert überwacht werden.

Starke Empfehlung, Konsens.

\section{EMPFEHLUNG 10B}

Die bei schwerer gastrointestinaler Blutung aufnehmende Intensivstation sollte aktuelle Qualitätsanforderungen erfüllen. Empfehlung, Konsens.

Es gibt einen breiten Konsens., dass Patienten mit gastrointestinaler Blutung adaptiert an den klinischen Zustand überwacht werden müssen [17]. Allerdings ist die Verfügbarkeit und Ausstattung (technisch/personell) von Notaufnahmestationen, Überwachungsstationen (mit Telemetrie), „Intermediate-Care-Stationen“ und Intensivstationen in Deutschland relativ heterogen, sodass es keine wissenschaftliche Evidenz gibt, für Patienten mit gastrointestinaler Blutung eine bestimmte Überwachungseinheit gegenüber anderen zu bevorzugen. Bei schwerer gastrointestinaler Blutung und hämodynamischer Instabilität sind selbstverständlich Strukturen zur Patientenversorgung erforderlich, die eine kontinuierliche Überwachung und unmittelbare Therapie (Volumenersatz, ggfs. Beatmung) erlauben.

Unter Beteiligung aller intensivmedizinisch tätigen Fachdisziplinen und der Fachkrankenpflege wurden im Jahr 2013 zehn Qualitätsindikatoren für deutsche Intensivstationen benannt [38], diese sind im Folgenden aufgeführt ( $\bullet$ Tab. 3).

\section{EMPFEHLUNG 11A}

Für die Versorgung von Patienten mit gastrointestinaler Blutung sollten auch außerhalb der Kernarbeitszeiten ein in der Notfallendoskopie erfahrener Arzt und in der Notfallendoskopie geschultes Assistenzpersonal in Rufbereitschaft sein. Empfehlung, starker Konsens.

\section{EMPFEHLUNG 11B}

Zudem sollen gerade bei Notfallendoskopien die strukturellen Empfehlungen der S3-Leitlinie „Sedierung in der gastrointestinalen Endoskopie“ strikt eingehalten werden.

Starke Empfehlung, starker Konsens. 
- Tab.3 Qualitätsanforderungen an Intensivstationen (nach [38]).

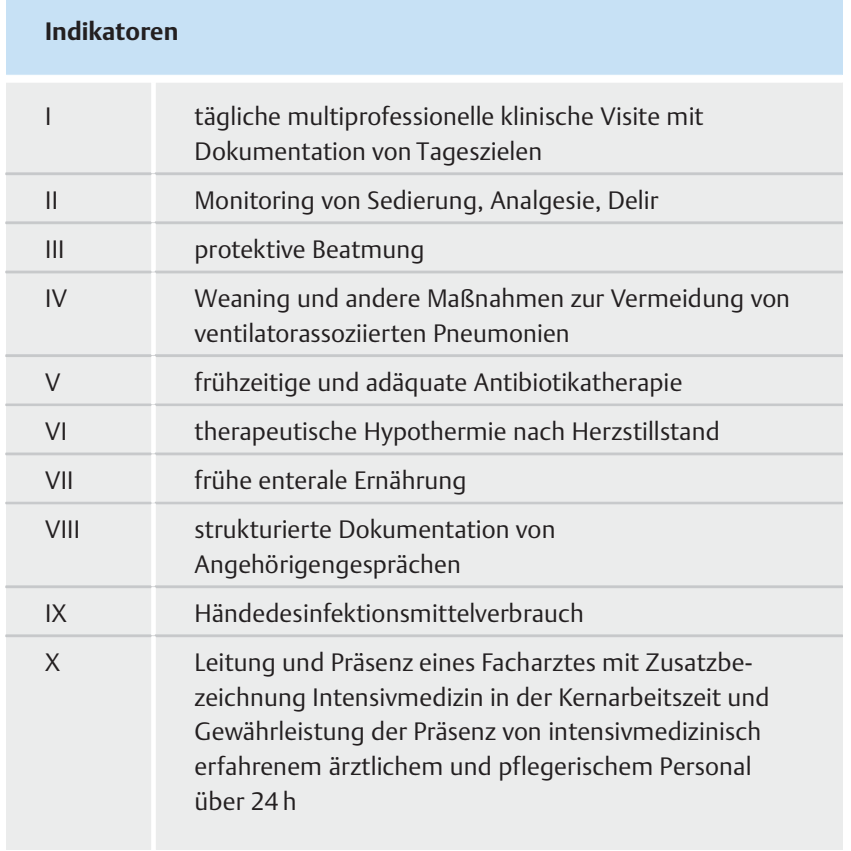

Um die geforderten Zeitintervalle bei variköser Blutung und bei hämorrhagischem Schock einhalten zu können, ist eine Rufbereitschaft, bestehend aus einer ärztlichen und einer pflegerischen Kraft, an sieben Tagen in der Woche rund um die Uhr erforderlich [1].

Interventionelle Endoskopien, inklusive Notfalluntersuchungen, sind mit einer höheren Rate an Sedierungszwischenfällen vergesellschaftet: In einer monozentrischen Registerstudie eines deutschen Zentrums bekamen über einen Zeitraum von sechs Jahren 6396 Patienten zur interventionellen Endoskopie eine Kombination aus Midazolam und Propofol, 3151 Patienten ausschließlich Propofol. Es traten 135 schwerwiegende Komplikationen auf, u. a. verstarben vier Patienten, bei neun Patienten musste eine endotracheale Intubation erfolgen. Als unabhängige Risikofaktoren wurden Alkohol- oder Drogenmissbrauch, eine hohe Propofol-Dosis und eben die Notfallendoskopie identifiziert [39]. Bei hämorrhagischem Schock bei gastrointestinaler Blutung liegt nach den Kriterien der American Society of Anesthesiologists (ASA) ein Grad IV (lebensbedrohliche Erkrankung) vor, sodass eine Endoskopie im hämorrhagischen Schock mittels Sedierung durch einen erfahrenen Arzt und mit einer adäquaten Überwachung begleitet werden muss [40].

\section{EMPFEHLUNG 12}

Bei unstillbarer bzw. rezidivierender nicht-variköser gastrointestinaler Blutung sollte zeitnah die Verlegung in ein Zentrum mit der Möglichkeit zur interventionellen Angiografie bzw. einer operativen Vorgehensweise erfolgen.

Empfehlung, starker Konsens.
Mittels endoskopischer Therapie kann sehr häufig eine suffiziente Hämostase erzielt werden (ggf. in Kombination mit medikamentöser Therapie). In einer 2014 veröffentlichten Arbeit konnte bei 411 von 428 Patienten (96\%) bei Ulkusblutung mittels Endoskopie eine Blutstillung erreicht werden, 69 Patienten benötigten eine zweite endoskopische Blutstillung, 18 Patienten eine dritte. Insgesamt konnten $82 \%$ der Rezidivblutungen endoskopisch beherrscht werden [41]. Außerdem stehen neue Techniken der endoskopischen Blutstillung zur Verfügung. Besonders vielversprechend scheinen Over-the-scope-Clips, neue Through-the-scopeClips und topisch anwendbare Substanzen zu sein, deren Wertigkeit später noch besprochen wird [42]. Dennoch stehen chirurgische und interventionell-angiografische Blutstillungsverfahren zur Verfügung, die nicht an jedem Standort vorgehalten werden können. Bei hohem Rezidivblutungsrisiko, Blutungsrezidiv oder nicht beherrschbarer Blutung sollte daher die Verlegung in ein Zentrum mit interventioneller Radiologie und erfahrener Viszeralchirurgie erfolgen.

\section{EMPFEHLUNG 13}

Bei unstillbarer bzw. rezidivierender variköser gastrointestinaler Blutung sollte zeitnah die Verlegung in ein Zentrum mit der Möglichkeit der Durchführung eines Rescue-TIPS bzw. frühelektiven TIPS erfolgen.

Empfehlung, starker Konsens.

Der Rescue-TIPS (transjugulärer intrahepatischer portosystemischer Shunt) kommt zum Einsatz bei endoskopisch nicht beherrschbarer Blutung (zwei endoskopische Interventionen innerhalb von $24 \mathrm{~h}$ ohne Blutstillung) bzw. bei frühzeitiger kreislaufrelevanter Rezidivblutung innerhalb von 5 Tagen [43]. Die Durchführung sollte an Zentren mit entsprechender Expertise erfolgen. Neben der effektiven medikamentösen pfortaderdrucksenkenden Therapie (Vasokonstriktoren: Terlipressin, etc.) stehen zur Überbrückung als lokale Maßnahmen zur Blutstillung Sengstaken-/Linton-Sonden oder selbstexpandierende Metallgitterstents (SEMS) zur Verfügung [44]. Als Ultima ratio können in Situationen rezidivierender variköser gastrointestinaler Blutung auch Shunt-Operationen oder, gerade bei begleitendem Leberversagen, eine Lebertransplantation (unter Beachtung der Indikationen und Kontraindikationen) erwogen werden [45].

\section{Initiale Stabilisierung, Volumen- und Transfusionsma- nagement}

\section{EMPFEHLUNG 14A}

Bei hämodynamischer Instabilität soll ein unverzüglicher Volumenersatz mittels kristalloider Lösungen erfolgen. Starke Empfehlung, starker Konsens. 


\section{EMPFEHLUNG 14B}

Wenn eine akute Hypovolämie allein mit Kristalloiden nicht ausreichend therapiert werden kann, können darüber hinaus Gelatine und Humanalbumin zum Einsatz kommen. Offene Empfehlung, starker Konsens.

Bei hämodynamischer Instabilität muss zunächst eine intravasale Hypovolämie ausgeglichen werden, um eine ausreichende Gewebsperfusion sicherzustellen und einem Multiorganversagen vorzubeugen [1]. Bei Patienten mit Schock steht daher die intensivmedizinische Kreislaufstabilisierung bei varikösen und nichtvarikösen Blutungen immer vor der Endoskopie [2]. Auch wenn es zum Volumenersatz bei gastrointestinalen Blutungen keine hochwertigen randomisierten Studien gibt, so reduzierte die rasche und frühe hämodynamische Stabilisierung bei Patienten mit akuter gastrointestinaler Blutung in einer Beobachtungs-Studie signifikant die Letalität $(p=0,04)$ und das Auftreten myokardialer Ereignisse $(p=0,04)$ gegenüber „konventionell“ behandelten Patienten [46]. Eine aktuelle Metaanalyse zum Volumenmanagement bei Traumapatienten mit hämorrhagischem Schock konnte allerdings keinen Vorteil einer frühen und aggressiven Volumentherapie belegen [47].

Zur Art der Volumenersatztherapie, d. h. kristalloide (Elektrolyt- oder niedermolekulare Kohlenhydratlösungen) vs. kolloidale (großmolekulare Flüssigkeiten) Lösungen, gibt es eine Vielzahl von Studien bei intensivmedizinisch versorgten Patienten, welche in der AMWF S3-Leitlinie „Intravasale Volumentherapie beim Erwachsenen“ (Registernummer 001 - 020) dargestellt werden [48]. Bei kritisch kranken Intensivpatienten war die Gabe von Kristalloiden im Vergleich zu Hydroxyethylstärke (HES) als Kolloid in mehreren randomisierten, kontrollierten Studien mit einem selteneren Auftreten eines Nierenversagens und besserem Überleben assoziiert [49]. Erneut muss einschränkend genannt werden, dass diese Studien nicht explizit Patienten mit akuter gastrointestinaler Blutung untersucht haben. Da durch Kolloide allerdings im allgemeinen eine raschere hämodynamische Stabilisierung gelingt [50], können Gelatine oder Humanalbumin zusätzlich zu kristalloiden Lösungen ebenfalls zum Einsatz kommen [51].

\section{EMPFEHLUNG 15}

Bei Patienten im hämorrhagischen Schock soll der Erfolg der intravasalen Volumenersatztherapie anhand klinischer (Vigilanz, Hautturgor), hämodynamischer (Herzfrequenz, Blutdruck, Schlagvolumen, dynamische Vorlastparameter), laborchemischer (Laktat) sowie ggfs. bildgebender (Echokardiografie) Befunde kontrolliert werden.

Starke Empfehlung, starker Konsens.

In der Initialphase des hämorrhagischen Schocks aufgrund einer gastrointestinalen Blutung dienen vor allem eine Normalisierung der Herzfrequenz und Stabilisierung des Blutdrucks (mittlerer arterieller Druck $>65 \mathrm{mmHg}$ ), einhergehend mit Normalisierung erhöhter Laktatspiegel in der bettseitigen Blutgasanalyse, als Indikatoren für einen erfolgreichen Ausgleich eines Volumenmangels. Gerade erhöhte Laktatspiegel (Laktat $>5 \mathrm{mmol} / \mathrm{l}$ ) haben in einer retrospektiven Auswertung intensivpflichtiger Patienten mit gastrointestinaler Blutung eine Assoziation zur Sterblichkeit [5]. Zur Steuerung der Volumentherapie bei erwachsenen, insbesondere bei beatmeten Intensivpatienten sollen Überwachungsverfahren, die eine Einschätzung der Volumenreagibilität anhand flussbasierter (Schlagvolumen) und/oder dynamischer Vorlastparameter (Schlagvolumenvariation, Pulsdruckvariation) erlauben, statischen Parametern (wie zentraler Venendruck, pulmonalarterieller Okklusionsdruck) vorgezogen werden [48]. Einzelheiten zum differenzierten Volumenmanagement im Schock sind der S3-Leitlinie „Intravasale Volumentherapie beim Erwachsenen“ (Registernummer 001 - 020) zu entnehmen [48].

\section{EMPFEHLUNG 16}

Bei unzureichendem Ansprechen auf eine Volumentherapie können im hämorrhagischen Schock passager Katecholamine zur Kreislaufstabilisierung eingesetzt werden.

Offene Empfehlung, starker Konsens.

Grundsätzlich ist die systemische Therapie mit Katecholaminen nicht geeignet, um einen hämorrhagischen Schock mit Hypovolämie dauerhaft zu behandeln. Dennoch kann es in Analogie zum septischen Schock notwendig werden, kurzzeitig den Kreislauf mit Katecholaminen wie Noradrenalin zu stabilisieren [52], bis die kausale Therapie (Volumen, Transfusion, Blutstillung) erfolgreich ist. Abgesehen von der besonderen Situation der varikösen Blutungen (Vasopressin- oder Somatostatin-Analoga als Mittel der Wahl) gibt es derzeit keine prospektiven Studien zum Einsatz von Vasokonstriktoren beim hämorrhagischen Schock aufgrund gastrointestinaler Blutungen [53].

\section{EMPFEHLUNG 17A}

Patienten mit vermuteter gastrointestinaler Blutung sollen allogene Erythrozytenkonzentrate erhalten, sodass der Hämoglobin-Wert zwischen 7-9g/dl stabilisiert wird. Starke Empfehlung, starker Konsens.

\section{EMPFEHLUNG 17B}

Bei einem Hämoglobin-Wert über $10 \mathrm{~g} / \mathrm{dl}$ und fehlenden klinischen Zeichen einer anämischen Hypoxie sollten keine Erythrozytenkonzentrate transfundiert werden. Empfehlung, starker Konsens. 


\section{EMPFEHLUNG 17C}

Bei massiver gastrointestinaler Blutung und hämorrhagischem Schock können Erythrozytenkonzentrate nach klinischer Einschätzung (unabhängig vom Hämoglobinwert) gegeben werden.

Offene Empfehlung, starker Konsens.

Eine große prospektive, allerdings monozentrische Studie hat den optimalen Transfusionstrigger bei 921 Patienten mit (vermuteter) gastrointestinaler Blutung (Meläna/Hämatemesis) untersucht. Ähnlich wie in anderen intensivmedizinischen Szenarien [54] war die restriktive Transfusionsstrategie (Transfusion nur, wenn Hämoglobin $[\mathrm{Hb}]<7 \mathrm{~g} / \mathrm{dl}$, Ziel-Hb $7-9 \mathrm{~g} / \mathrm{dl}$ ) der liberalen Transfusionsstragie (Transfusion bei $\mathrm{Hb}<9 \mathrm{~g} / \mathrm{dl}$, Ziel-Hb $9-11 \mathrm{~g} / \mathrm{dl}$ ) bezüglich der Gesamtletalität überlegen (HR 0,55) [55]. Außerdem gab es weniger Rezidivblutungen bei restriktiver Transfusionsindikation (HR 0,68) [55]. Auch in Subgruppenanalysen (z. B. Zirrhose) ergab sich kein Vorteil für eine liberale Transfusionsstrategie [55]. Eine multizentrische Studie aus Großbritannien hat eine Cluster-Analyse für 936 Patienten mit gastrointestinaler Blutung durchgeführt, in der Krankenhäuser mit restriktiver (Transfusion bei $\mathrm{Hb}<8 \mathrm{~g} / \mathrm{dl}$ ) und mit liberaler (bei $\mathrm{Hb}<10 \mathrm{~g} / \mathrm{dl}$ ) Transfusionsstrategie verglichen wurden [56]. Bei restriktiver Transfusionsindikation kam es insgesamt zu weniger Transfusionen pro Patient, wohingegen es keinen Unterschied bei klinischen Endpunkten (Reblutung, Überleben, Thrombembolien) gab [56]. Allerdings gab es in dieser Studie in der Subgruppe der Patienten mit koronarer Herzerkrankung einen Trend ( $p=0,11)$ zu erhöhter Letalität (12 vs. $3 \%$ ) bei restriktiver Transfusionsstrategie, sodass in dieser Subgruppe möglicherweise ein Transfusionstrigger von $\mathrm{Hb}<10 \mathrm{~g} / \mathrm{dl}$ sinnvoll sein könnte [56].

Einschränkend muss zudem angeführt werden, dass in diesen prospektiven Studien Patienten mit schwerer gastrointestinaler Blutung (hämodynamische Instabilität, sichtbare schwerste Blutung) und einigen Komorbiditäten ausgeschlossen waren [55, 56]. In Situationen mit klinisch offensichtlicher massiver Blutung erscheint daher eine unmittelbare Transfusion nach klinischer Einschätzung und ohne spezifischen Transfusionstrigger sinnvoll.

Die Querschnitts-Leitlinie der Bundesärztekammer (BÄK) zur Therapie mit Blutkomponenten und Plasmaderivaten [57] räumt insbesondere der klinischen Einschätzung (z. B. physiologische Transfusionstrigger wie Tachykardie, Hypotension, EKG-Ischämie, Laktazidose) großen Raum ein; gemäß dieser Querschnittsleitlinie kann (bei niedrigerer 2C-Evidenz) auch eine Transfusion bei $\mathrm{Hb}$ Werten zwischen $8-10 \mathrm{~g} / \mathrm{dl}$ erfolgen, wenn klinische Hinweise auf eine anämische Hypoxie bestehen.

\section{Medikamentöse Therapie}

\section{EMPFEHLUNG 18}

Bei vermuteter nicht-variköser oberer gastrointestinaler Blutung sollen Protonenpumpenhemmer als intravenöse Bolusgabe (80 mg i. v.) verabreicht werden.

Starke Empfehlung, starker Konsens.
Die präendoskopische intravenöse Bolusgabe eines Protonenpumpenhemmers (PPI), z. B. Pantoprazol $80 \mathrm{mg}$ i. v., wird in den verfügbaren Leitlinien zu nicht-varikösen Blutungen empfohlen $[1,2,58,59]$. Dies wird gestützt durch eine Metaanalyse aus sechs randomisierten kontrollierten Studien mit insgesamt 2223 Patienten, in denen die präendoskopische PPI-Gabe zwar nicht die Rezidivblutungsrate oder Letalität, aber signifikant die Rate von Blutungsstigmata (aktive Blutung, Blutkoagel oder Gefäßstumpf) in der Endoskopie (37,2\% vs. 46,5\%; OR 0,67) und die Notwendigkeit endoskopischer Interventionen (8,6 vs. 11,7\%; OR 0,68) reduzierte [60]. Allerdings darf durch die PPI-Gabe die Endoskopie nicht verzögert werden [1].

\section{EMPFEHLUNG 19}

Bei vermuteter nicht-variköser oberer gastrointestinaler Blutung sollten Protonenpumpenhemmer intravenös, entweder als wiederholte Bolusgaben oder als kontinuierliche Applikation mittels Perfusor, bis zur Endoskopie fortgesetzt gegeben werden.

Empfehlung, starker Konsens.

In den meisten Studien der erwähnten Metaanalyse, die einen Vorteil für die präendoskopische PPI-Gabe nachgewiesen hat, wurden typischerweise nach einer initialen PPI-Bolusgabe bis zur Endoskopie kontinuierlich PPI über Perfusorsysteme $(8-10 \mathrm{mg} / \mathrm{h})$ fortgesetzt, sodass davon auszugehen ist, dass PPI bis zur endoskopischen Versorgung verabreicht werden sollten $[1,60]$. Aus vergleichenden Analysen zur fortgesetzten PPI-Gabe bei nachgewiesener schwerer Ulkusblutung ist bekannt, dass wiederholte PPI-Bolusgaben (meist 3-mal 40 mg pro Tag) gegenüber einer kontinuierlichen PPI-Gabe über Perfusor (zumeist 240 mg/24h) gleichwertig sind [61].

\section{EMPFEHLUNG 20}

Bei vermuteter variköser oberer gastrointestinaler Blutung können Protonenpumpenhemmer verabreicht werden.

Offene Empfehlung, starker Konsens.

Diese sollten aber nicht ohne genaue Überprüfung der Indikation als Dauermedikation fortgesetzt werden.

Empfehlung, starker Konsens.

Wie bereits oben ausgeführt, ist präendoskopisch die Unterscheidung zwischen variköser und nicht-variköser Blutungsquelle schwierig; auch Patienten mit Leberzirrhose haben zu ca. 50\% eine nicht-variköse Blutungsursache bei oberer gastrointestinaler Blutung, sodass die Initialtherapie mit einem PPI möglich erscheint [6]. Bei Patienten mit variköser oberer gastrointestinaler Blutung sind PPI allerdings nicht oder nur wenig wirksam [62]. Die Rolle von PPI in der akuten Varizenblutung oder in der Prophylaxe von Rezidivblutungen nach endoskopischer Intervention ist derzeit noch nicht abschließend geklärt $[16,63]$.

Es gibt aus einer prospektiven Beobachtungsstudie allerdings klare Hinweise, dass die dauerhafte Gabe von PPI bei Patienten 
- Tab. 4 Ergebnisse einer randomisierten Studie zum Einsatz vasoaktiver Substanzen bei akuter Varizenblutung, die drei Studienarme zeigten keine signifikanten Unterschiede (nach [72]).

\begin{tabular}{|c|c|c|c|}
\hline & Terlipressin & Somatostatin & Octreotid \\
\hline & $N=261$ & $N=259$ & $N=260$ \\
\hline $\begin{array}{l}\text { Dosierung Bolus } \\
\text { (vor Endoskopie!) }\end{array}$ & $2 \mathrm{mg}$ Bolus i. v. & $250 \mu \mathrm{g}$ Bolus i.v. & $50 \mu \mathrm{g}$ Bolus i. v. \\
\hline Dosierung über $5 \mathrm{~d}$ & $1 \mathrm{mg}$ alle $6 \mathrm{~h}$ i.v. für $5 \mathrm{~d}$ & $250 \mu \mathrm{g} / \mathrm{h}$ i. v. für $5 \mathrm{~d}$ & $25 \mu \mathrm{g} / \mathrm{h}$ i. v. für $5 \mathrm{~d}$ \\
\hline $\begin{array}{l}\text { aktive Blutung bei erster } \\
\text { Endoskopie }\end{array}$ & $43,7 \%$ & $44,4 \%$ & $43,5 \%$ \\
\hline Therapieerfolg Tag 5 & $86,2 \%$ & $83,4 \%$ & $83,8 \%$ \\
\hline Rezidivblutung & $3,4 \%$ & $4,8 \%$ & $4,4 \%$ \\
\hline Letalität & $8,0 \%$ & $8,9 \%$ & $8,8 \%$ \\
\hline
\end{tabular}

mit Leberzirrhose mit einer erhöhten Letalität (HR 2,36) assoziiert ist [64]. Unter langfristiger PPI-Gabe treten bei Patienten mit Leberzirrhose möglicherweise gehäuft spontan bakterielle Peritonitiden auf [65]. Daher sollte keine unkritische Fortsetzung einer (Hochdosis-)PPI-Therapie bei Patienten mit Leberzirrhose als Dauermedikation erfolgen.

\section{EMPFEHLUNG $21 \mathrm{~A}$}

Bei begründetem Verdacht auf eine akute Varizenblutung soll eine intravenöse Therapie mit einem Vasokonstriktor (Terlipressin, Somatostatin oder Octreotid) noch vor der Endoskopie begonnen werden.

Starke Empfehlung, starker Konsens.

\section{EMPFEHLUNG 21B}

Bei Bestätigung der Diagnose sollte die VasokonstriktorTherapie noch 3-5 Tage fortgesetzt werden.

Empfehlung, starker Konsens.

Zwei Gruppen von vasoaktiven Substanzen werden erfolgreich in der Therapie der akuten Varizenblutung eingesetzt: Vasopressin und seine Analoga (teils gemeinsam mit Nitraten) sowie Somatostatin und seine Analoga. Terlipressin ist ein Vasopressin-Analogon, welches sowohl systemisch als auch in den Mesenterialgefäßen zu einer Vasokonstriktion führt und so den Pfortaderfluss reduziert. Somatostatin und das synthetische Somatostatin-Analogon Octreotid führen zu einer Vasokonstriktion im Splanchnikusgebiet über Hemmung der Glukagon-vermittelten splanchnischen Vasodilatation. Diese Vasokonstriktiva reduzieren letztlich den Pfortaderfluss und damit die Durchblutung der Umgehungs- kreisläufe, zusätzlich stabilisieren sie den Kreislauf und verbessern die Nierendurchblutung $[2,62]$. Randomisierte Studien und Metaanalysen zeigten klar, dass der Einsatz vasoaktiver Substanzen bei der akuten Varizenblutung mit einer reduzierten Letalität und einer besseren Blutungskontrolle assoziiert ist $[66,67]$, insbesondere in Kombination mit endoskopischen Maßnahmen [28, 68 70]. Daher soll bei begründetem Verdacht auf eine Varizenblutung (d. h. obere gastrointestinale Blutung bei bekannter oder vermuteter Leberzirrhose, anamnestisch bekannte Varizen) die Gabe einer vasoaktiven Substanz bereits vor der Endoskopie erfolgen [16, 62].

Am häufigsten wird in Deutschland Terlipressin in der Dosierung von 1-2 mg als intravenöse Bolusgabe eingesetzt, welche alle 4- 6 Stunden innerhalb der ersten 72 Stunden wiederholt wird [2]. Die optimale Dauer der Gabe vasoaktiver Substanzen ist nicht abschließend geklärt, möglicherweise reichen auch kürzere Zeiträume (z. B. 24 Stunden) aus [71].

Eine große randomisierte prospektive Studie mit 780 Patienten hat die Effekte von Terlipressin, Somatostatin und Octreotid in der akuten Varizenblutung verglichen: In Bezug auf die untersuchten Endpunkte (Blutungskontrolle bei erster Endoskopie, Reblutungsrate, Letalität) zeigten die verschiedenen Regimes keine signifikanten Unterschiede ( $\triangleright$ Tab.4) [72]. In der Terlipressin-Gruppe traten allerdings signifikant häufiger Hyponatriämien als in den übrigen Gruppen auf [72]. In internationalen Empfehlungen werden alle drei Substanzen derzeit als gleichwertig angesehen $[16,62]$.

\section{EMPFEHLUNG 22A}

Bei begründetem Verdacht auf eine akute Varizenblutung soll eine intravenöse Antibiotika-Therapie noch vor der Endoskopie begonnen werden.

Starke Empfehlung, starker Konsens. 


\section{EMPFEHLUNG 22B}

Bei Bestätigung der Diagnose sollte die Antibiotika-Therapie noch 5- 7 Tage fortgesetzt werden.

Empfehlung, starker Konsens.

Bei Patienten mit Leberzirrhose und akuter Varizenblutung kann die frühzeitige Gabe von Antibiotika mit breitem gram-negativen Wirkspektrum, z. B. Ceftriaxon 2 g/24h oder Ciprofloxacin 2-mal $500 \mathrm{mg} / 24 \mathrm{~h}$, das Auftreten klinisch relevanter bakterieller Infektionen verhindern. In einer Metaanalyse aus 12 Plazebo-kontrollierten Studien reduzierte die Antibiotika-Therapie signifikant die Letalität ( $R R=0,79,95 \% \mathrm{KI} 0,63-0,98)$, bakterielle Infektionen $(R R=0,43,95 \% \mathrm{KI} 0,19-0,97)$ und die frühe Reblutungsrate $(R R=0,53,95 \%$ KI 0,38-0,74) [73]. Da sowohl das Infektions- als auch das Letalitätsrisiko bei Patienten mit kompensierter Leberzirrhose (Child-Pugh Stadium A) gering(er) ist, wird aber diskutiert, ob bei diesen Patienten auf eine Antibiotika-Gabe verzichtet werden kann [16]. Die Wahl des Antibiotikums sollte patientenspezifische (z. B. Vorbehandlungen) als auch Umgebungs-spezifische (z. B. Keim- und Resistenzspektrum im Krankenhaus) Spektren berücksichtigen [16]; gerade aus Südeuropa ist eine hohe Rate Chinolon-resistenter Darmkeime berichtet worden, sodass hier Drittgenerations-Cephalosporine bevorzugt werden [74]. Die optimale Dauer der Antibiotika-Gabe ist nicht abschließend geklärt, typischerweise wird die präinterventionell begonnene Therapie für 5 - 7 Tage fortgesetzt. Eine Anpassung der Antibiose gemäß eingehender mikrobiologischer Befunde ist selbstverständlich („Tarragona-Strategie“) [75].

\section{EMPFEHLUNG 23}

Bei vermuteter schwerer oberer gastrointestinaler Blutung sollte Erythromycin $250 \mathrm{mg}$ i.v. einmalig als Kurzinfusion 30 - 120 Minuten vor der Indexendoskopie gegeben werden, um die Beurteilbarkeit des oberen Gastrointestinaltrakts zu verbessern.

Empfehlung, Konsens.

Insbesondere wenn der Magen noch mit Altblut, Blutkoageln oder Speiseresten gefüllt ist, kann bei einem Teil der Patienten in der ersten Endoskopie die Blutungsquelle nicht oder nur unzureichend identifiziert werden [76]. Daher erscheint die Gabe eines Prokinetikums vor der (Notfall-)Endoskopie sinnvoll, um die Beurteilbarkeit des oberen Gastrointestinaltrakts zu verbessern [2]. Die Einmalgabe des Motilin-Rezeptor-Agonists Erythromycin vor Endoskopie wurde in fünf Metaanalysen beurteilt [59, $77-80$ ], in denen sich insgesamt positive Effekte auf Endpunkte wie Lokalisierbarkeit der Blutungsquelle oder Notwendigkeit einer erneuten Endoskopie zeigten. In der größten dieser Metaanalysen ( $n=558$ Patienten, sechs Studien) verbesserte die Erythromycin-Infusion (gegenüber Plazebo oder keiner Gabe) vor Endoskopie bei oberer gastrointestinaler Blutung signifikant die Beurteilbarkeit der Magenschleimhaut (OR 3,43; 95 \% KI 1,81-6,50), Notwendigkeit einer zweiten Endoskopie (OR 0,47; 95 \% KI 0,26-0,83), Transfusionsbedarf $(p=0,04)$ und Liegedauer im Krankenhaus $(P<0,01)$ [79]. Die Einmal-Gabe von Erythromycin (250 mg i. v. als Kurzinfusion) war in den Metaanalysen sicher und gut verträglich, zudem ist diese Maßnahme kosteneffektiv [80]. Allerdings ist Erythromycin nicht in dieser Indikation in Deutschland zugelassen, sodass es sich um einen „off label“-Gebrauch handelt [2]. Wichtige Kontraindikationen gegen die Gabe von Erythromycin sind bekannte Allergien gegen Makrolid-Antibiotika und eine verlängerte QT-Zeit [1]. Prinzipiell besteht das Risiko lebensbedrohlicher Herzrhythmusstörungen („Long-QT-Syndrom“, ventrikukäre Arrhythmien), gerade, wenn Erythromycin gemeinsam mit anderen Medikamenten eingesetzt wird, welche die QT-Zeit verlängern (z. B. Chinolone, Psychopharmaka, Antiarrhythmika).

Metoclopramid ist in dieser Situation weniger gut untersucht, scheint aber ebenfalls die Beurteilbarkeit in der Notfall-Endoskopie zu verbessern [81]. Für Metoclopramid besteht insbesondere bei längerer Gabe das Risiko schwerer neurologischer Nebenwirkungen [1], es kann aber als Alternative bei Kontraindikationen gegen Erythromycin eingesetzt werden.

\section{Atemwegsmanagement}

\section{EMPFEHLUNG 24A}

Bei (vermuteter) schwerer oberer gastrointestinaler Blutung kann zur Verringerung des Aspirationsrisikos eine endotracheale Intubation vor der Notfallendoskopie erfolgen.

Offene Empfehlung, starker Konsens.

\section{EMPFEHLUNG 24B}

Bei klinisch deutlich erhöhtem Aspirationsrisiko (schwere Hämatemesis, Enzephalopathie) sollte eine endotracheale Intubation vor der Endoskopie erwogen werden.

Empfehlung, starker Konsens.

Die endotracheale Intubation vor der Notfallendoskopie soll gerade bei Patienten mit schwerer oberer gastrointestinaler Blutung das Aspirationsrisiko verringern. Zum Erfolg einer „prophylaktischen Intubation“ („Schutzintubation“) gibt es allerdings keine randomisierten prospektiven Studien; retrospektive Auswertungen konnten keinen Vorteil in Bezug auf patientenrelevante Endpunkte (z. B. Aspirationspneumonie, Letalität) nachweisen [8284]. In einer aktuellen größeren amerikanischen retrospektiven Studie wurden 144 Patienten mit prophylaktischer Intubation vor Gastroskopie mit 221 Patienten mit gastrointestinaler Blutung ohne Intubation verglichen. Auch in gematchten Populationen von jeweils 100 Patienten pro Gruppe mit ähnlichem Risikoprofil gab es mehr unerwünschte kardiopulmonale Ereignisse (vor allem Pneumonien und Kreislaufschock) bei Patienten mit prophylaktischer Intubation [85]. In einer retrospektiven Studie traten Aspirationspneumonien ebenfalls häufiger bei Patienten mit prophylaktischer Intubation auf [82], was allerdings auch durch einen 
Selektions-Bias (Patienten mit schwererer Blutung wurden häufiger prophylaktisch intubiert) erklärt werden könnte. Da es derzeit keine hochwertigen Studien gibt, die einen Vorteil der prophylaktischen Intubation nachweisen, kann hierzu keine generelle Empfehlung ausgesprochen werden (vgl. hierzu auch S3-Leitlinie "Sedierung in der gastrointestinalen Endoskopie“ 2014, AWMFRegister-Nr. 021/014) [40]. Bei Patienten mit klinisch erkennbar deutlich erhöhtem Aspirationsrisiko, d. h. insbesondere bei schwerer Hämatemesis, vermuteter aktiver Ösophagusvarizenblutung, bereits bestehender Vigilanzminderung oder hepatischer Enzephalopathie, sollte allerdings eine endotracheale Intubation vor der Endoskopie erwogen werden, weil hier von einem sehr hohen Aspirationsrisiko auszugehen ist [1].

\section{Aufklärung und Einwilligung}

\section{EMPFEHLUNG 25A}

Vor Durchführung intensivmedizinischer oder endoskopischer Maßnahmen sollte geklärt werden, welches Therapieziel erreicht werden kann und welchem der Patient oder an seiner Stelle dessen gesetzlicher Vertreter zustimmt.

Empfehlung, Konsens.

\section{EMPFEHLUNG 25B}

Patient oder gesetzlicher Vertreter sollen über Diagnose, Prognose, geplante Maßnahmen und Behandlungsalternativen einschließlich der damit verbundenen Risiken verständlich informiert werden; bei lebensrettenden Maßnahmen sind zwischen Aufklärung und Durchführung keine festen Fristen einzuhalten.

Starke Empfehlung, starker Konsens.

\section{EMPFEHLUNG $25 C$}

Ist der Patientenwille in der zur Verfügung stehenden Zeit nicht erhebbar, soll zum Wohl des Patienten und dem mutmaßlichen Patientenwillen entsprechend entschieden werden.

Starke Empfehlung, starker Konsens.

Zur Aufklärung vor endoskopischen Eingriffen sei auf die S2k-Leitlinie „Qualitätsanforderungen in der gastrointestinalen Endoskopie“ (AWMF-Register Nr. 021 - 022) verwiesen [2]. Ein wesentlicher Bestandteil der Patient-Arzt-Beziehung ist die Vereinbarung von Therapiezielen. Diese Aufgabe geht dem Einsatz jeder Diagnostik- und Therapiemöglichkeit voraus. Besonders für Patienten mit zweifelhafter Prognose ist zu klären, welches Therapieziel im Spektrum zwischen kurativ orientierter, maximal möglicher Lebensverlängerung und palliativ orientiertem Zulassen des Sterbens verfolgt werden soll [86].
Im Notfall und bei unzureichender Information stehen zunächst Sicherung und Aufrechterhaltung der Vitalfunktionen nach den in dieser Leitlinie dargelegten Kriterien mit dem Therapieziel „Lebenserhaltung“ im Vordergrund.

Parallel zu den dazu notwendigen Maßnahmen geht es darum, sich ein möglichst umfassendes Bild vom Patienten zu verschaffen und neben Anamnese und Befunden patientenbezogene, individuelle Faktoren (z. B. bekannte Behandlungspräferenzen, persönliche Werthaltungen, religiöse Einstellung, Alter) zu berücksichtigen und die Therapieziele entsprechend anzupassen.

Die erarbeiteten Therapieziele müssen dem Patienten oder seinem Stellvertreter ausführlich mitgeteilt und mit ihm besprochen werden, um seine Entscheidung einzuholen. Dabei sind Diagnose, Prognose, zu ergreifende Maßnahmen mit ihren Risiken und alle wichtigen Umstände zu erörtern und zu dokumentieren [Patientenrechtegesetz $\S \S 630 c-f$ BGB: https://www.gesetze-im-internet.de/bgb/_630c.html].

Die Entscheidung über das anzustrebende Therapieziel trifft dann der Patient oder sein Stellvertreter. Das angestrebte Therapieziel muss dokumentiert und im Behandlungsverlauf regelmäßig überprüft werden.

Eine Änderung des Therapieziels ist erforderlich, wenn der Patient oder sein Stellvertreter dies wünscht oder wenn sich relevante medizinische Veränderungen ergeben.

Gibt es Meinungsdifferenzen oder Unsicherheiten zu fachlichen Fragen, ist auf die Möglichkeit hinzuweisen, eine Zweitmeinung durch einen anderen Experten einzuholen. Bestehen ethische Entscheidungskonflikte, ist eine Ethikberatung, z. B. durch ein Klinisches Ethikkomitee, zu empfehlen [http://www.ethikkomitee.de/].

\section{Arbeitsgruppe 2: Gerinnungsstatus und Antikoagulanzien}

Für elektive Eingriffe werden viele Problemstellungen wie laborchemische Analysen vor elektiver Endoskopie, das Vorgehen bei Patienten mit angeborener oder erworbener nicht medikamentös bedingter Hämostasestörung oder das Pausieren von Thrombozytenaggregationshemmung (TAH) bzw. Antikoagulation umfassend in der S2k-Leitlinie „Qualitätsanforderungen in der gastrointestinalen Endoskopie, AWMF-Register Nr. 021 - 022, Erstauflage 2015“ beantwortet. Dort werden folgende Themen abgehandelt, die im Hinblick auf die Vermeidung von gastrointestinalen Blutungen auch in den Geltungsbereich der vorliegenden Leitlinie fallen: Blutungsrisiko bei bestimmten endoskopischen Prozeduren, Medikation mit TAH, Heparinen, oralen Antikoagulanzien (VitaminK-Antagonisten und DOAKs) inklusive deren Halbwertszeiten und empfohlenen Zeiträumen der Pausierung, sowie Empfehlungen zur Einnahme von gerinnungshemmender Medikation in Abhängigkeit vom Risikoprofil bestimmter endoskopischer Eingriffe. Diese Empfehlungen behalten ihre Gültigkeit und sollen zur Vermeidung von Redundanzen in der vorliegenden Leitlinie nicht erneut aufgeführt werden [87].

Daher erfolgen die nachfolgenden Empfehlungen im Kontext der akuten gastrointestinalen Blutung bei Patienten mit angebo- 
rener oder erworbener, nicht medikamentös-bedingter Hämostasestörung oder unter laufender Therapie mit TAH oder Antikoagulanzien.

\section{Wie häufig treten gastrointestinale Blutungen unter Thrombozytenaggregation oder Antikoagulation auf?}

\section{AUSSAGE 2A}

Die Therapie mit einem Thrombozytenaggregationshemmer erhöht das Risiko für eine gastrointestinale Blutung. Starker Konsens.

In einer Metaanalyse von über 100000 Patienten aus prospektiven Studien zeigte sich das Risiko von Blutungen insgesamt (OR 1,70) sowie von schweren oder relevanten Blutungen (OR 1,31; NNH 73) unter Acetylsalicylsäure (ASS) erhöht [88]. In einer weiteren Metaanalyse wurde die auf ASS zurückzuführende Steigerung von gastrointestinalen Blutungen auf jährlich 0,13\% für Majorblutungen und 0,12\% für Minorblutungen kalkuliert [89]. Das relative Risiko lag bei 2,22 für schwere gastrointestinale Blutungen sowie 1,23 für tödliche Blutungen.

Randomisierte prospektive Studien zu P2Y12-Antagonisten im Vergleich zu Plazebo existieren nicht; in einer zu ASS randomisierten Studie (CAPRIE-Study) ergab sich für Clopidogrel ein leicht vermindertes Risiko (ASS vs. Clopidogrel RR 1,45) gastrointestinaler Blutungen im Vergleich zu ASS [89]. Andere Studien weisen ein eher leicht erhöhtes Blutungsrisiko gegenüber ASS aus [90 - 92].

Die Gabe eines Antikörpers gegen GP IIb/IIla bei Patienten mit Myokardinfarkt erhöht ebenfalls das Blutungsrisiko [93].

\section{AUSSAGE 2B}

Das Risiko gastrointestinaler Blutungen ist unter dualer Thrombozytenaggregationshemmung höher als bei Monotherapie.

Starker Konsens.

Bei dualer Plättchenaggregationshemmung steigt das Risiko gastrointestinaler Blutungen in der ASS/Clopidogrel-Gruppe relativ zur ASS-Monotherapiegruppe auf eine HR von 2,7 an [94]. Die dreifache (Triple-) Thrombozytenaggregations-Therapie (TAT, Zugabe von Cilostazol zu ASS und Clopidogrel) und die konventionelle duale Thrombozytenaggregationshemmung (ASS und Clopidogrel) wurden in einer Metaanalyse verglichen. Das Gesamtblutungsrisiko war in beiden Gruppen gleich (2,39 vs. 2,04\%, $p=0,49$ ), das Risiko von gastrointestinalen Nebenwirkungen (gastrointestinale Blutungen wurden nicht gesondert ausgewiesen) war signifikant höher in der TAT Gruppe $(\mathrm{OR}=2,46)$ [95].

\section{AUSSAGE 2C}

Das Risiko für eine gastrointestinale Blutung unter Antikoagulation ist erhöht. Das Risiko unterscheidet sich zwischen Vitamin-K-Antagonisten und direkt wirkenden oralen Antikoagulanzien (DOAKs) nicht.

Starker Konsens.

Die gastrointestinale Blutung ist eine typische Komplikation der oralen Antikoagulation mit Vitamin-K-Antagonisten [96, 97]. In einer finnischen Kohortenstudie von über 100000 Patienten lag das Risiko, aufgrund einer gastrointestinalen Blutung hospitalisiert zu werden, bei 2,3\% pro Patientenjahr zu Beginn und 0,9\% pro Patientenjahr im weiteren Verlauf einer Therapie mit oralen Antikoagulanzien. Dabei war das Risiko einer Blutung in den ersten 30 Tagen nach Einleitung der Antikoagulation am höchsten [98]. 30 bis $40 \%$ aller Blutungsereignisse bei oral antikoagulierten Patienten sind gastrointestinalen Ursprungs [99], $6 \%$ dieser Blutungen enden tödlich [100].

Das Risiko, eine gastrointestinale Majorblutung zu erleiden, hängt, wahrscheinlich durch Komorbiditäten, Begleitmedikationen und Länge der Therapiedauer bedingt, von der Indikation zur Antikoagulation ab; bei Patienten mit Vorhofflimmern liegt die Inzidenz bei 2,5\%, nach Lungenembolie bei 0,5\% [99].

Eine 2013 publizierte Metaanalyse zu DOAKs, die 43 prospektiv randomisierte Studien einschloss, wies im Vergleich zu Vitamin-KAntagonisten ein leicht erhöhtes Risiko klinisch relevanter gastrointestinaler Blutungen nach (OR 1,45) [101]. Das Risiko war bei den Indikationen Vorhofflimmern (OR 1,21) und venösen Thrombosen (OR 1,59) leicht, bei der Indikation akutes Koronarsyndrom jedoch deutlich (OR 5,21) erhöht. Limitierend wurden eine recht große Heterogenität der eingeschlossenen Studien und das jeweilige Studiendesign, das auf die Effektivität der DOAKs im Vergleich zur Standardtherapie ausgelegt gewesen war, beschrieben [101].

In einer jüngeren Metaanalyse von insgesamt 23 prospektiven Phase III-Studien, die die Wirkung von direkt wirkenden oralen Antikoagulanzien (Apixaban, Dabigatran, Doxaban und Rivaroxaban) auf das Risiko von gastrointestinalen Majorblutungen verglichen, zeigte sich gepoolt aus fünf kontrollierten randomisierten Studien bei den Indikationen Vorhofflimmern (RR 1.08) und venösen Thrombembolien kein Unterschied zu Vitamin-K-Antagonisten [99]. Auch im Vergleich (allerdings nur eine Studie) zur Acetylsalicylsäure (RR 0,78), zu niedermolekularen Heparinen allein (RR 1,42, $95 \% \mathrm{KI} 0,55$-3,71, I2=7\%; 8 RCTs), zur sequenziellen Therapie von niedermolekularem Heparin und Vitamin-K-Antagonisten RR 0,77, 95 \% KI 0,49 - 1,21, 12 = 43 \%; 7 RCTs) oder Plazebo (RR 1,48, $95 \%$ KI 0,15-14,84, I2 = $21 \%$; 2 RCTs) zeigten die DOAKs kein erhöhtes Risiko für Major-gastrointestinale Blutungen. 


\section{AUSSAGE 2 D}

Das Risiko erhöht sich bei Kombination der Antikoagulation mit einem Thrombozytenaggregationshemmer oder bei Kombination mit einer dualen antithrombozytären Therapie erheblich.

Starker Konsens.

Bei oral antikoagulierten Patienten, die sich zudem einer perkutanen Koronarintervention unterziehen müssen, ist eine zusätzliche Therapie mit einem oder zwei Thrombozytenaggregationshemmern indiziert. Der Vorteil im Hinblick auf das kardiovaskuläre Ergebnis wird jedoch mit einem erhöhten Blutungsrisiko erkauft. In der „STent Anticoagulation Restenosis Study“ fand sich in der Gruppe derjenigen Patienten, die eine duale Therapie mit oraler Antikoagulation plus ASS erhielten, mit 6,2 vs. 1,8\% eine deutlich höhere Gesamtblutungsrate im Vergleich zu denjenigen, die nur ASS einnahmen [102]; der Anteil der gastrointestinalen Blutungen wurde jedoch nicht gesondert ausgewiesen.

Unter Tripletherapie mit ASS, Clopidogrel und Enoxaparin im Rahmen einer Kurzzeitbehandlung aufgrund eines akuten Koronarsyndroms wurde in einer retrospektiven Analyse von $666 \mathrm{~Pa}$ tienten bei $2,7 \%$ eine gastrointestinale Blutung innerhalb von 30 Tagen dokumentiert [103].

In einer retrospektiven dänischen Registerstudie mit 118606 Patienten lagen die Blutungsereignisse bei der Tripletherapie bei $15,7 \%$ pro Patientenjahr, bei der dualen Therapie mit Vitamin-KAntagonist und Clopidogrel bei 13,9\% [90]. Im Vergleich zur Monotherapie mit Vitamin-K-Antagonist ergaben sich Risikoerhöhungen von 3,1-fach für Vitamin-K-Antagonist/Clopidogrel und 3,7-fach für die Tripletherapie. Dabei trat eine gastrointestinale Blutung zu 5,1\% pro Jahr auf, was einer Steigerung von 5,38-fach im Vergleich zur oralen Antikoagulation als Monotherapie entspricht. Dieselbe Arbeitsgruppe konnte in einer weiteren Registerstudie zeigen, dass das Risiko einer Blutung nach Myokardinfarkt unter Tripletherapie etwa 1,4-fach höher lag als unter der Kombination von Vitamin-K-Antagonist und Thrombozytenaggregationshemmer. Zu beachten ist, dass der Anteil gastrointestinaler Blutungen bei den tödlichen gegenüber den nicht-tödlichen Ereignissen überrepräsentiert war (45,3 vs. 33,8\%) [92].

In einer prospektiven Studie, die Blutungen als primäres Zielkriterium definiert hatte (WOEST-Trial), lag die Inzidenz von Blutungsereignissen sogar noch deutlich höher [104], möglicherweise bedingt durch die systematische Erfassung. Bei Patienten mit dualer Therapie wurden innerhalb eines Jahres Blutungsepisoden bei $19,4 \%$, bei Patienten mit Tripletherapie bei $44,4 \%$ notiert. Bei 2,9 bzw. 8,8\% aller Patienten traten gastrointestinale Blutungen auf. Der Anteil von Majorblutungen zeigte sich über alle Ereignisse statistisch nur in Abhängigkeit von dem zugrundegelegten Score (TIMI, GUSTO, BARC) partiell bzw. tendenziell signifikant, wobei der Schweregrad der gastrointestinalen Blutung nur eingeschränkt durch die aufgeführten Scores abgebildet wird.

\section{Welche Laborwerte sollten vor der Intervention vorliegen? Welche Gerinnungsstörungen sollten vor Endoskopie korrigiert werden? Wie sollte korrigiert werden?}

\section{EMPFEHLUNG 26A}

Eine notwendige endoskopische Intervention soll bei akuter gastrointestinaler Blutung unabhängig von vorliegenden Laborwerten begonnen werden.

Starke Empfehlung, starker Konsens.

\section{EMPFEHLUNG 26B}

Die Gerinnungsparameter (Thrombozyten, Quick/INR, PTT) sollten zeitnah bestimmt werden. Bei Hinweisen auf Gerinnungsstörungen sollte situationsbezogen eine erweiterte Gerinnungsdiagnostik erfolgen.

Empfehlung, starker Konsens.

\section{EMPFEHLUNG 26C}

Blutungsrelevante Gerinnungsstörungen sollten unabhängig vom Zeitpunkt der Endoskopie korrigiert werden. Empfehlung, starker Konsens.

\section{EMPFEHLUNG 26 D}

Patienten mit einer bekannten hereditären oder erworbenen Blutgerinnungsstörung sollten im Rahmen einer akuten Blutung entsprechend ihrer Gerinnungsstörung substituiert werden.

Empfehlung, starker Konsens.

Hinsichtlich der Empfehlungen zur Bestimmung des Blutbildes wird auf der Empfehlung 2b der Leitlinie verwiesen. Studien zur Routinetestung von Gerinnungsparametern bei Patienten ohne Anamnese für eine prädisponierende Erkrankung oder ein Blutungsereignis vor chirurgischen oder endoskopischen Eingriffen zeigten keine Korrelation zu dem tatsächlichen Auftreten von Blutungsereignissen [105]. Eine gerinnungsphysiologische Diagnostik im Rahmen einer akuten gastrointestinalen Blutung sollte bei Vorliegen von anamnestischen Faktoren und Vorerkrankungen erfolgen, die auf eine zugrundeliegende Gerinnungsstörung hinweisen. Dies beinhaltet folgende Faktoren:

- positive Blutungsanamnese nach vorherigen Eingriffen

- internistische Vorerkrankungen, die mit einem erhöhten Blutungsrisiko einhergehen können: vaskuläre hämorrhagische Diathese (Morbus Osler, Ehlers-Danlos-Syndrom, MarfanSyndrom) 
- angeborene Thrombozytopathien

- angeborene plasmatische Gerinnungsstörung (Von-Willebrand-Syndrom, angeborener Faktor VIII-(FVIII)- oder Faktor IX-(FIX)-Mangel)

- erworbene Thrombozytenfunktionsstörung und/oder erworbene plasmatische Gerinnungsstörung i.R. von Leber-, Nierenerkrankungen, prolongierter biliärer Obstruktion, prolongierter Antibiotikaeinnahme, Malnutritution oder myeloproliferativen Erkrankungen

Neben der Bestimmung des Quick-Wertes/INR und der PTT ist die Fibrinogenbestimmung zu empfehlen, da bei einem Fibrinogen $<1 \mathrm{~g} / \mathrm{l}$ der Quick/INR aufgrund der Methodik (koagulometrische Messung) pathologisch gemessen wird. Ein Mangel an Fibrinogen tritt deutlich häufiger erworben als angeboren auf. Mögliche Pathomechanismen beinhalten neben medikamentös induzierten Effekten vor allem einen vermehrten Verbrauch im Rahmen einer Hyperfibrinolyse oder einer disseminierten intravasalen Gerinnung, Verlust und Dilution bei schweren Blutungen oder sehr selten Antikörperbildung gegen Fibrinogen. Die Gabe von PPSB (beinhaltet die Faktoren II - Prothrombin, VII - Prokonvertin, X Stuart-Proper-Faktor und IX) wäre dann, bei unzureichendem Fibrinogen, ohne Effekt. Insbesondere im Rahmen einer massiven Blutung bei Leberzirrhose ist die zusätzliche Fibrinogenbestimmung daher unbedingt zu empfehlen. Die lokale Blutstillung sollte jedoch unabhängig von dem Vorliegen aktueller Laborwerte erfolgen.

Empfehlung zum Vorgehen bei Patienten mit angeborener oder erworbener nicht medikamentös bedingter Hämostasestörung

In der Literatur finden sich nur wenige systematische Beobachtungs- und keine kontrollierten Interventionsstudien, die sich im Rahmen akuter gastrointestinaler Blutungen spezifisch mit dem hämostaseologischen Vorgehen bei Patienten mit angeborener Blutungsneigung beschäftigen [106].

Aus diesem Grund orientieren sich die Empfehlungen vorrangig an den Querschnittsleitlinien der Bundesärztekammer zur Therapie mit Blutkomponenten und Plasmaderivaten aus dem Jahr 2008 [107], in denen für Patienten mit Hämophilie oder von-Willebrand-Syndrom das allgemeine Vorgehen zur Blutungsprophylaxe und -therapie bei invasiven oder operativen Eingriffen geregelt ist, der Leitlinie der World Federation of Hemophilia [108] sowie an der an den meisten hämostaseologischen Zentren gängigen klinischen Praxis (good clinical practice). Dabei unterscheidet sich der Umgang bei den verschiedenen Störungen nicht von denjenigen, die für die elektive Endoskopie ausführlich in der Leitlinie „Qualitätsanforderungen in der gastrointestinalen Endoskopie“ dargelegt wurden [109]. Eine Zusammenfassung der jeweiligen therapeutischen Optionen bietet $>$ Tab. $\mathbf{5}$.

Im Rahmen einer akuten gastrointestinalen Blutung bei Patienten mit angeborener oder erworbener nicht-medikamentös bedingter Hämostasestörung sollten die konkreten Maßnahmen zur akuten Blutungstherapie nach Möglichkeit in Rücksprache mit einem in der Behandlung von Hämostasestörungen erfahrenen Zentrum erfolgen. Der angeborene oder erworbene Hämostase- defekt sollte im Fall einer akuten Blutung durch eine entsprechende Therapie korrigiert werden. Insbesondere bei instabilen Patienten sollte die endoskopische Intervention unabhängig von der Klärung der Hämostasestörung und deren therapeutischer Beeinflussung von der klinischen Situation geleitet werden.

Im Folgenden werden Empfehlungen zu den wesentlichen angeborenen und erworbenen Gerinnungsstörungen gegeben, im Einzelfall ist eine Rücksprache zu konkreten Maßnahmen mit einem entsprechend erfahrenen Zentrum dennoch unerlässlich.

\section{Hepatische Koagulopathie}

Die Beurteilung des Blutungsrisikos bei Patienten mit chronischer Lebererkrankung und hepatischer Koagulopathie sollte primär auf der Grundlage anamnestischer und klinischer Daten erfolgen. Ergänzend können hämostaseologische Parameter wie Blutungszeit, Thrombozytenzahl und plasmatische Gerinnungstests (Quick/INR und aPTT) herangezogen werden.

Patienten mit Leberzirrhose haben ein erhöhtes Blutungsrisiko. Zudem sind bei den meisten Zirrhosepatienten die routinemäßig angeforderten Labortests zur Beurteilung der primären (Thrombozytenzahl und Blutungszeit) und sekundären Hämostase (Quick/INR und aPTT) pathologisch verändert. Bisher konnten jedoch weder der kausale Zusammenhang zwischen normabweichenden Hämostasetests einerseits und hämorrhagischen Komplikationen andererseits noch der Nutzen einer Korrektur derselben belegt werden [110-112].

Sehr wahrscheinlich wird das Blutungsrisiko von Zirrhosepatienten wesentlich durch andere Faktoren beeinflusst wie z. B. portale Hypertension, endotheliale Dysfunktion, Einschwemmung heparinähnlicher Substanzen im Rahmen bakterieller Infektionen oder Niereninsuffizienz, sodass neben einer gründlichen Anamnese der bestmöglichen Modifikation dieser Faktoren besondere Bedeutung zukommt $[111,112]$. Der Einsatz von Tranexamsäure im Rahmen der akuten Blutung kann aufgrund der häufig vorherrschenden Hyperfibrinolyse empfohlen werden $(\triangleright$ Tab. 5).

\section{Thrombozytenaggregation/Antikoagulation: Wann sollte/sollte nicht pausiert werden? Bei welchen Erkrankungen sollte umgestellt werden?}

\section{AUSSAGE 3}

Eine Notfallendoskopie zur Blutstillung ist auch unter Thrombozytenaggregationshemmung oder Antikoagulanzien möglich und sinnvoll.

Starker Konsens.

Es existieren keine Daten über eine optimale INR, um die endoskopische Notfalltherapien durchführen zu können. Sicher scheint die endoskopische Blutstillung bei einer INR von 1,5-2,5, wie in einer kleinen kontrollierten Studie von Choudari bei mit Warfarin antikoagulierten Patienten gezeigt wurde [113]. Für Patienten mit supratherapeutischer INR gibt es keine prospektiven Daten zur Sicherheit einer Endoskopie, doch waren in einer retrospektiven 
- Tab.5 Therapeutische Optionen bei Gerinnungsstörungen und gastrointestinalen Blutungen.

\begin{tabular}{|c|c|c|c|}
\hline Gerinnungsstörung & Therapie & Handelsname & Dosierung (Bemerkungen) \\
\hline \multirow[t]{3}{*}{$\begin{array}{l}\text { von- Willebrand-Syndrom } \\
\text { (vWS) Typ } 1\end{array}$} & $\begin{array}{l}\text { 1-Desamino-8-D-Arginin-Vasopressin } \\
\text { (DDAVP) }\end{array}$ & $\begin{array}{l}\text { z. B. Minirin }{ }^{\circledR} \\
\text { parenteral }\end{array}$ & $\begin{array}{l}0,3 \mu \mathrm{g} / \mathrm{kg} \text { KG über } 30 \mathrm{~min} \text {. (ca. } 3 / 4 \text { Ampulle von } \\
\text { jeweils } 4 \mu \mathrm{g} \text { pro } 10 \mathrm{~kg} \text { KG in } 50-100 \mathrm{ml} \mathrm{NaCl} \mathrm{0,9 \% )} \\
\text { oder als Nasenspray (Octostim }{ }^{\circledR} \text { ) } 1 \text { Sprühstoß pro } \\
\text { Nasenloch (Gesamtdosis } 300 \mu \mathrm{g})\end{array}$ \\
\hline & Tranexamsäure & z. B. Cyklokapron ${ }^{\circledR}$ & $\begin{array}{l}\text { Erstgabe } 10 \mathrm{mg} / \mathrm{kg} \text { KG i. v.; Fortführung mit } 1 \mathrm{~g} / 8 \mathrm{~h} \\
\text { möglich }\end{array}$ \\
\hline & $\begin{array}{l}\text { Nur in begründeten Ausnahmefällen, z. B. } \\
\text { bei Unwirksamkeit oder Unverträglichkeit } \\
\text { von DDAVP, ist bei Patienten mit VWS Typ } \\
1 \text { die Substitution eines VWF-haltigen } \\
\text { (FVIII) Konz. }\end{array}$ & $\begin{array}{l}\text { z. B. Haemate }{ }^{\circledR} \mathrm{P} \text {, } \\
\text { Wilate }^{\circledR} \text {, Willfact }\end{array}$ & $\begin{array}{l}\text { initiale Dosierung } 30 \text { bis } 60 \text { IE/kg KG, Zielspiegel für } \\
\text { von Willebrandfaktor, Ristocetin-Cofaktor und FVIII } \\
>50 \% \\
\text { (fast reines VWF-Konzentrat ohne nennenswerte } \\
\text { FVIII-Anteil, für Behandlung einer akuten Blutung } \\
\text { allein nicht geeignet) }\end{array}$ \\
\hline $\begin{array}{l}\text { vWS Typ } 2 \\
(10-30 \%)\end{array}$ & $\begin{array}{l}\text { Substitution eines VWF-haltigen (FVIII) } \\
\text { Konz. }\end{array}$ & $\begin{array}{l}\text { z. B. Haemate }{ }^{\circledR} \mathrm{P} \text {, } \\
\text { Wilate }^{\circledR} \text {, Willfact }\end{array}$ & $\begin{array}{l}\text { initiale Dosierung } 30 \text { - } 60 \text { IE/kg KG, Zielspiegel für } \\
\text { von Willebrandfaktor, Ristocetin-Cofaktor und FVIII } \\
>50 \% \\
\text { (fast reines VWF-Konzentrat ohne nennenswerte } \\
\text { FVIII-Anteil, für Behandlung einer akuten Blutung } \\
\text { allein nicht geeignet) }\end{array}$ \\
\hline $\begin{array}{l}\text { vWS Typ } 3 \\
(1-5 \%)\end{array}$ & $\begin{array}{l}\text { Substitution eines VWF-haltigen (FVIII) } \\
\text { Konz. plus Tranexamsäure }\end{array}$ & $\begin{array}{l}\text { z. B. Haemate }{ }^{\circledR} \text { P, } \\
\text { Wilate }^{\circledR} \text {, Willfact }\end{array}$ & $\begin{array}{l}\text { initiale Dosierung } 30 \text { - } 60 \mathrm{IE} / \mathrm{kg} \mathrm{KG} \text {, Zielspiegel für } \\
\text { von Willebrandfaktor, Ristocetin-Cofaktor und FVIII } \\
>50 \% \\
\text { (fast reines VWF-Konzentrat ohne nennenswerte } \\
\text { FVIII-Anteil, für Behandlung einer akuten Blutung } \\
\text { allein nicht geeignet) }\end{array}$ \\
\hline erworbenes vWS (AvWS) & individuell nach Grundkrankheit & & $\begin{array}{l}\text { (Substitution des VWF-Konz. häufig ohne } \\
\text { anhaltenden Effekt) }\end{array}$ \\
\hline \multirow[t]{2}{*}{ Hämophilie A } & $\begin{array}{l}\text { DDAVP (Desmopressin) plus } \\
\text { Tranexamsäure }\end{array}$ & $\begin{array}{l}\text { z. B. Minirin } \\
\text { parenteral }\end{array}$ & $\begin{array}{l}0,3 \mu \mathrm{g} / \mathrm{kg} \text { KG über } 30 \mathrm{~min} \text {. (ca. } 3 / 4 \text { Ampulle von } \\
\text { jeweils } 4 \mu \mathrm{g} \text { pro } 10 \mathrm{~kg} \text { KG in } 50-100 \mathrm{ml} \mathrm{NaCl} 0,9 \% \text { ) } \\
\text { oder als Nasenspray (Octostim }{ }^{\circledR} \text { ) } 1 \text { Sprühstoß pro } \\
\text { Nasenloch (Gesamtdosis } 300 \mu \mathrm{g})\end{array}$ \\
\hline & $\begin{array}{l}\text { FVIII-Konz. bei schwerer und } \\
\text { mittelschwerer Hämophilie A }\end{array}$ & $\begin{array}{l}\text { Rekomb. und plasma- } \\
\text { tische FVIIII-Konz. }\end{array}$ & 25 bis 40 IE/kg KG; Zielspiegel FVIII > 50\% \\
\hline Hämophilie B & FIX-Konz. plus Tranexamsäure & $\begin{array}{l}\text { Rekomb. und plasma- } \\
\text { tische FIX-Konz. }\end{array}$ & $40-60$ IE/kg KG; Zielspiegel Faktor IX > 50\% \\
\hline \multirow[t]{2}{*}{ Hemmkörper-hämophilie } & Rekomb. aktivierter FVII plus & NovoSeven $^{\circledR}$ & $90 \mu \mathrm{g} / \mathrm{kg}$ KG / $2-3 \mathrm{~h}$ \\
\hline & aktiviertes Prothrombinkomplexkonz. & FEIBA NF & $\begin{array}{l}50-100 \mathrm{IE} / \mathrm{kg} \mathrm{KG} / 8-12 \mathrm{~h} \text { (max. Tagesdosis } \\
200 \mathrm{IE} / \mathrm{kg} \text { ) }\end{array}$ \\
\hline \multirow[t]{2}{*}{ FVII-Mangel } & $\begin{array}{l}\text { Restaktivität > } 30 \% \text { und unauffällige } \\
\text { Blutungsanamnese: ggf. Gabe von } \\
\text { Tranexamsäure }\end{array}$ & z. B. Cyklokapron ${ }^{\circledR}$ & $\begin{array}{l}\text { Erstgabe } 10 \mathrm{mg} / \mathrm{kg} \text { KG i. v.; Fortführung mit } 1 \mathrm{~g} / 8 \mathrm{~h} \\
\text { möglich }\end{array}$ \\
\hline & $\begin{array}{l}\text { Restaktivität }<30 \% \text { zusätzlich } \\
\text { Gerinnungsfaktorkonz. }\end{array}$ & $\begin{array}{l}\text { Immuseven }{ }^{\circledR} \\
\text { NovoSeven }^{\circledR}\end{array}$ & 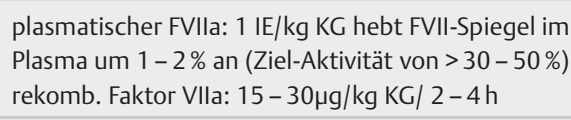 \\
\hline angeborener FV-Mangel & Tranexamsäure gefrorenes Frischplasma & & Zielspiegel FV > $30 \%$ \\
\hline angeborener FXIII-Mangel & $\begin{array}{l}\text { Tranexamsäure } \\
\text { FXIII-Konz. }\end{array}$ & Fibrogammin & $\begin{array}{l}1 \mathrm{IE} / \mathrm{kg} \text { KG hebt FXIII-Spiegel im Plasma um } 2 \% \text { an } \\
\text { (Ziel-Aktivität > 50\%) }\end{array}$ \\
\hline \multirow[t]{2}{*}{$\begin{array}{l}\text { angeborene Thrombozy- } \\
\text { ten-funktionsstörungen }\end{array}$} & DDAVP & $\begin{array}{l}\text { z. B. Minirin } \\
\text { parenteral }\end{array}$ & $\begin{array}{l}0,3 \mu \mathrm{g} / \mathrm{kg} \text { KG über } 30 \mathrm{~min} \text {. (ca. } 3 / 4 \text { Ampulle von je- } \\
\text { weils } 4 \mu \mathrm{g} \text { pro } 10 \mathrm{~kg} \mathrm{KG} \text { in } 50-100 \mathrm{ml} \mathrm{NaCl} \mathrm{0,9 \% )} \\
\text { oder als Nasenspray (Octostim }{ }^{\circledR} \text { ) } 1 \text { Sprühstoß pro } \\
\text { Nasenloch (Gesamtdosis } 300 \mu \mathrm{g} \text { ) }\end{array}$ \\
\hline & $\begin{array}{l}\text { bei ausbleibendem Therapieerfolg: } \\
\text { Thrombozytenkonz., Tranexamsäure, } \\
\text { rekomb. FVIla }\end{array}$ & z. B. Cyklokapron ${ }^{\circledR}$ & $\begin{array}{l}\text { Erstgabe } 10 \mathrm{mg} / \mathrm{kg} \mathrm{KG} \mathrm{i.} \mathrm{v.;} \mathrm{Fortführung} \mathrm{mit} 1 \mathrm{~g} / 8 \mathrm{~h} \\
\text { möglich }\end{array}$ \\
\hline
\end{tabular}


- Tab. 5 (Fortsetzung)

\begin{tabular}{|c|c|c|c|}
\hline Gerinnungsstörung & Therapie & Handelsname & Dosierung (Bemerkungen) \\
\hline $\begin{array}{l}\text { hepatische } \\
\text { Koagulopathie }\end{array}$ & $\begin{array}{l}\text { Tranexamsäure } \\
\text { bei ausbleibendem Therapieerfolg PPBS, } \\
\text { gefrorenes Frischplasma, ggf. Thrombo- } \\
\text { zytenkonz. }\end{array}$ & & $\begin{array}{l}\text { bei andauernder Blutung sinnvolle Zielwerte: } \\
\text { Thrombozyten }>50 / \mu \mathrm{l} \\
\text { Fibrinogen }>1 \mathrm{~g} / \mathrm{l} \\
\text { Quick }>50 \%\end{array}$ \\
\hline
\end{tabular}

Analyse die Rate an Rezidivblutungen, Transfusionspflicht und Todesfällen im Krankenhaus bei einer entgleisten INR von > 4 ähnlich wie bei einer INR im therapeutischen Bereich [114]. Die Autoren schlussfolgern, dass angesichts der hohen Rate an positiven endoskopischen Befunden in deren Kollektiv die Notfallendoskopie auch bei supratherapeutischer INR durchgeführt werden sollte. Andererseits war in einer landesweiten Untersuchung in England eine INR von > 1,5 der häufigste Grund für eine fehlende endoskopische Blutungskontrolle [115]. Größere prospektive Daten hierzu existieren aber ebenso wenig wie vergleichbare Daten zur Sicherheit einer Notfallendoskopie unter DOAKs. In der Erfahrung der Autoren erübrigt sich in den meisten Fällen lebensbedrohlicher akuter gastrointestinaler Blutungen eine solche Diskussion, da eine intensivmedizinische Therapie mit evtl. periinterventioneller Antagonisierung von Antikoagulanzien bzw. Thrombozytentransfusion unter Thrombozytenaggregationshemmung abhängig vom Schweregrad der Blutung erwogen wird (hämorrhagischer Schock vs. signifikante Blutung ohne hämodynamische Beeinträchtigung vs. Minorblutung) und in der Regel parallel zur oder unmittelbar vor der Notfallendoskopie verläuft.

\section{EMPFEHLUNG 27}

Das Risiko bzw. der Nutzen einer Fortführung der Thrombozytenaggregationshemmung oder Antikoagulation nach Blutstillung im Rahmen einer Notfallendoskopie sowie der Zeitpunkt der Wiederaufnahme sollen interdisziplinär abgewogen werden gegen a) den Schweregrad der Blutung und das Risiko einer Rezidivblutung sowie b) das thromboembolische Risiko des Patienten.

Starke Empfehlung, starker Konsens.

Diese bewusst allgemein gehaltenen Aussagen gelten sowohl für den Einsatz von Thrombozytenaggregationshemmern als auch für Antikoagulanzien während einer akuten gastrointestinalen Blutung, denn trotz deren hoher Inzidenz gibt es wenig evidenzbasierte Daten zum periinterventionellen Management der Substanzen, insbesondere Studien der höheren Evidenzklassen. Das „American College of Chest Physicians“ z. B. empfiehlt eine Pausierung von Antikoagulanzien im Falle einer gastrointestinalen Majorblutung, gibt aber keine Empfehlungen zum optimalen Zeitpunkt der Wiederaufnahme. Im Folgenden soll dennoch zwischen
Thrombozytenaggregationshemmern und Antikoagulanzien differenziert werden.

\section{EMPFEHLUNG 28A}

Bei Patienten, die Thrombozytenaggregationshemmer nur zur Primärprophylaxe erhalten, sollte die Medikation bei einer akuten gastrointestinalen Blutung periinterventionell pausiert werden.

Empfehlung, starker Konsens.

\section{EMPFEHLUNG 28B}

Bei Patienten mit mittlerem bis hohem Risiko für arterielle Thrombosen sollte die Medikation mit Thrombozytenaggregationshemmern in Abhängigkeit vom Schweregrad der Blutung zwar periinterventionell pausiert, aber nach endoskopischer Blutstillung so rasch wie möglich wiederaufgenommen werden.

Empfehlung, starker Konsens.

\section{EMPFEHLUNG 28C}

Ein kardiologisches oder neurologisches Konsil zur gemeinsamen Entscheidungsfindung kann durchgeführt werden. Offene Empfehlung, starker Konsens.

Bei Patienten mit schweren gastrointestinalen Blutungen unter Therapie mit Thrombozytenaggregationshemmern besteht grundsätzlich die Option, die Thrombozytenaggregationshemmer zu pausieren und ggf. zusätzlich Thrombozytenkonzentrate zu transfundieren. Es existieren wenig evidenzbasierte Daten darüber, ob und wann dies sinnvoll ist und wann eine Thrombozytenaggregationshemmung wiederaufgenommen werden soll. Daher beruhen Empfehlungen anderer Fachgesellschaften meist auf Expertenmeinungen. Bei einer akut lebensbedrohlichen gastrointestinalen Blutung muss die Thrombozytenaggregationshemmung gestoppt werden, bis eine sichere Hämostase erzielt wurde. Bei der Entscheidung zur Aufhebung des Effekts der Thrombozytenaggregationshemmer mittels Thrombozytenkon- 
zentraten muss das Risiko von Ischämien gegen das Risiko der weiteren Blutung unter gehemmter Plättchenaggregation abgewogen werden; die Transfusion erwies sich in mehreren Studien zur Plättchenaggregation als effektiv, auch bei doppelter Hemmung durch ASS und Clopidogrel (Übersicht in einem Editorial; [116]), konnte aber für den PAR-1 Antagonisten Vorapaxar nicht gezeigt werden [117].

Bei den meisten Patienten mit gastrointestinaler Blutung ist eine Wiederaufnahme der Thrombozytenaggregationshemmung möglich, sobald die endoskopische Blutstillung gesichert ist. Sowohl ein Konsens.uspapier der ACCF/ACG/AHA von 2008 [118] als auch die aktuelle Leitlinie der ASGE [119] empfehlen im jeweiligen Einzelfall die enge Abstimmung zwischen Gastroenterologen und den die Thrombozytenaggregationshemmung verschreibenden Spezialisten (insbes. Kardiologen). Wenngleich es für diese Empfehlung keine Daten gibt, erscheint dies im klinischen Alltag sinnvoll und praktikabel, insbesondere da das Risiko für die Entwicklung von Koronarstentthrombosen unter den zahlreichen Modalitäten für Nicht-Spezialisten kaum zu überblicken ist (siehe Antikoagulanzien). Der Zeitpunkt der Wiederaufnahme sollte nach dem kardiovaskulären Risiko stratifiziert werden. Allgemein überwiegt das Risiko bei Patienten mit niedrigem kardiovaskulärem Risiko (z. B. solche, die Thrombozytenaggregationshemmer zur Primärprophylaxe einnehmen) den Nutzen einer frühen Wiederaufnahme. Dagegen wurden bei Patienten, die ASS zur Sekundärprophylaxe einnahmen, in einer randomisierten prospektiven Studie von Sung et al. unmittelbar nach endoskopischer Stillung einer Ulkusblutung entweder ASS oder Plazebo verabreicht ( $n=156$ ); dabei gab es keine signifikanten Unterschiede in der Rate an Rezidivblutungen. Eine verzögerte Wiederaufnahme von ASS war allerdings mit einer erhöhten 30-Tage-Mortalität verbunden [120]. Daher sollte die Wiederaufnahme der Thrombozytenaggregationshemmung so rasch wie möglich erfolgen. Zum Management von Patienten mit akuter gastrointestinaler Blutung unter doppelter Thrombozytenaggregationshemmung existieren keine vergleichbar guten Daten; praktikabel scheint auch hier eine Pausierung bei lebensbedrohlicher Blutung und eine Wiederaufnahme, sobald endoskopisch die Hämostase gesichert wurde. Besondere Vorsicht ist bei der Wiederaufnahme von Ticagrelor geboten, da die Plättchenhemmung nicht durch Transfusion aufgehoben werden kann. Die frühe Fortsetzung der doppelten Thrombozytenaggregationshemmung nach Blutstillung sollte insbesondere bei Patienten mit „Bare Metal Stents“ innerhalb der ersten 4 Wochen sowie bei „Drug eluting Stents“ innerhalb der ersten 1-6 Monate nach Koronarintervention erwogen werden, da diese Patienten besonders häufig zu Stentthrombosen neigen. Das Risiko einer erhöhten Rate an kardiovaskulären Ereignissen konnte auch in einer Metaanalyse mit insgesamt über 50000 Patienten demonstriert werden. Das periinterventionelle Pausieren von ASS zur Sekundärprophylaxe resultierte in einer 3-fach höheren Rate an kardialen Ereignissen. Bei Patienten mit Koronarstents war das Risiko sogar noch deutlich höher. Die in diesen Studien eingeschlossenen Patienten waren sehr heterogen und nicht auf gastrointestinale Blutungen fokussiert [121]. Ähnliches zeigt eine zweite Metaanalyse mit perioperativer Pausierung von ASS; diese Studie ist von hoher Relevanz, da die kardiovaskulären Ereignisse teils innerhalb von 5 Tagen nach ASS-Pausierung auftraten [122].
Wenngleich eine Übertragbarkeit dieser Studien auf akute gastrointestinale Blutungen formal nicht zulässig ist, suggerieren sie dennoch, dass die frühe Wiederaufnahme der Thrombozytenaggregationshemmung nach endoskopischer Blutstillung grundsätzlich sinnvoll ist, zumal selbst bei erneuter gastrointestinaler Blutung die endoskopische Blutstillung meist gut möglich ist.

\section{EMPFEHLUNG 29}

Die Antikoagulation mit Vitamin-K-Antagonisten oder DOAKs sollte postinterventionell abhängig vom Schweregrad der Blutung und dem Thromboembolierisiko zum frühestmöglichen Zeitpunkt wiederaufgenommen werden, in der Regel aber zum Zeitpunkt der Krankenhausentlassung bzw. nach ca. 7 Tagen. Dies gilt nicht für Patienten mit mechanischen Herzklappen und hohem Thromboembolierisiko.

Empfehlung, starker Konsens.

Während mehrere kleine retrospektive Studien variable Ergebnisse erzielten, konnte in einer größeren retrospektiven Studie an 442 Patienten unter Warfarin gezeigt werden, dass die frühe Wiederaufnahme der Antikoagulation nach einer akuten gastrointestinalen Blutung sinnvoll ist [123].

Nach Bereinigung für klinische Parameter (z. B. Schweregrad der Blutung, Transfusionspflicht) konnte gezeigt werden, dass die Wiederaufnahme von Warfarin innerhalb von 90 Tagen nach einer gastrointestinalen Blutung mit einer ca. 10-fach geringeren Rate an thromboembolischen Ereignissen (0,4 bzw. 5,5\%; HR $0,05)$ und einer ca. 3-fach geringeren Mortalität (5,8 bzw. 20,3\%; HR 0,31) assoziiert war (mit der frühesten Thrombose innerhalb von 8 Tagen), ohne das Risiko einer Rezidivblutung signifikant zu steigern (10 bzw. 5,5\%, HR 1,31).

Eine große retrospektive Analyse von 1329 Patienten mit gastrointestinaler Blutung bestätigt bei etwas anderem Design diese Ergebnisse [124]. Die Wiederaufnahme der Antikoagulation mit Warfarin war mit einer reduzierten Rate an Thromboembolien (HR 0,71) und signifikant reduzierten Mortalität (HR 0,67) bei nicht signifikant erhöhter Blutungsrate (HR 1,18) assoziiert. Bei Stratifizierung nach der Dauer der Warfarin-Pausierung war die Wiederaufnahme innerhalb von 7 Tagen mit einer geringen Mortalität und Thromboembolierate im Vergleich zur Wiederaufnahme nach 30 Tagen assoziiert, ebenfalls ohne erhöhte Rezidivblutungsrate.

Die erste prospektive Analyse zu diesem Thema wurde 2015 von Sengupta et al. publiziert [125]. Hier wurde das Ergebnis bei 197 Patienten mit einer gastrointestinalen Blutung unter Antikoagulation erfasst. Die Ursache der gastrointestinalen Blutungen war sehr heterogen (größter Anteil mit 37\% „nicht identifizierte Blutungsquelle“); $74 \%$ der Patienten erhielten Warfarin, $13 \%$ DOAK, $8 \%$ Enoxaparin und 6\% unfraktioniertes Heparin. Bei $58 \%$ der Patienten war die Indikation zur Antikoagulation Vorhofflimmern. Bei $39 \%$ war die Antikoagulation bei Entlassung noch pausiert, bei den verbleibenden $61 \%$ wiederaufgenommen worden. Die Entscheidung zur Pausierung oder Wiederaufnahme war durch den entlassenden Arzt erfolgt, wobei die mediane Dauer 
des Krankenhausaufenthaltes in den beiden Gruppen 5 bzw. 6 Tage betrug. Die Rate an Bluttransfusionen, Intensivaufenthalten und die Ätiologie der zur Antikoagulation führenden Erkrankung waren in beiden Gruppen ähnlich. Innerhalb von 90 Tagen traten bei $4 \%$ thromboembolische Ereignisse auf, und 14 Patienten wurden wegen einer Rezidivblutung wiederaufgenommen. In der Patientengruppe mit fortgesetzter Antikoagulation zum Zeitpunkt der Krankenhausentlassung war die Rate an thromboembolischen Ereignissen signifikant geringer (HR 0,12), während die Rate an Rezidivblutungen nicht signifikant erhöht war (HR 2,17; $p=0,10)$. Die Autoren schlussfolgern, dass die Vorteile einer Wiederaufnahme der Antikoagulation zum Zeitpunkt der Krankenhausentlassung im Hinblick auf thromboembolische Ereignisse das Risiko einer Rezidivblutung überwiegen.

Für DOAKs existieren bislang keine vergleichbaren Daten, sodass nur angenommen werden kann, dass der Vorteil einer frühen Wiederaufnahme nach endoskopischer Hämostase ähnlich ist wie bei o. g. Studien zu Vitamin-K-Antagonisten. Aufgrund des frühen Wirkungseintritts von DOAKs und der bislang nicht standardisierten Antagonisierung (s. u.) ist hier besondere Vorsicht geboten.

Wenngleich die drei größeren genannten Studien nahelegen, dass eine frühe Wiederaufnahme der Antikoagulation mit Vitamin-K-Antagonisten sinnvoll ist, gibt es keine Daten darüber, wann der optimale Zeitpunkt zur Wiederaufnahme ist, also wann der Nutzen bezüglich des Thromboembolierisikos das Risiko der Rezidivblutung überwiegt. Für DOAKs gibt es hierzu ebenfalls keine Daten. Es wäre denkbar, für die Stratifizierung auch den Schweregrad der Blutung mittels verschiedener Blutungs-Scores hinzuzuziehen. Evidenzbasiert ist dies nicht. Daher bleibt die Entscheidung über den Zeitpunkt der Wiederaufnahme stets eine Einzelfallentscheidung, die in der Regel zwischen Gastroenterologen und Kardiologen sowie Patienten gemeinsam getroffen werden sollte; dies gilt insbesondere aufgrund der für den Gastroenterologen kaum zu überblickenden Thromboembolierisiken bei vielfältigen Indikationen für Antikoagulanzien, wie eine italienische Umfrage unter Ärzten zum generellen Management von Warfarin-assoziierter gastrointestinaler Blutung zeigte [126].

Während bei endoskopisch identifizierter Blutungsquelle und Hämostase die Entscheidung zur Wiederaufnahme der Antikoagulation leichterfällt, stellt der Zeitpunkt bei endoskopisch nicht identifizierter Blutungsquelle ein weiteres Problem dar, sodass auch hier nur im Einzelfall anhand der Blutungsschwere, des HbVerlaufs und des individuellen Thromboembolierisikos entschieden werden kann. Bei nicht sicherer endoskopischer Blutstillung kann eine Kontroll-Endoskopie sinnvoll sein.

\section{EMPFEHLUNG $30 \mathrm{~A}$}

Bei Vorhofflimmern und Pausieren der Antikoagulation sollte eine Überbrückungstherapie („,bridging“) nicht routinemäßig durchgeführt werden.

Empfehlung, starker Konsens.

\section{EMPFEHLUNG 30B}

Die Wiederaufnahme der Antikoagulation sollte unter Abwägung des Rezidivblutungs- und Thrombose-/Embolierisikos erfolgen.

Empfehlung, starker Konsens.

Es existieren keine evidenzbasierten Daten, die das Blutungsrisiko gegenüber dem Thromboembolierisiko untersuchen. Das Risiko bei mechanischen und frühen biologischen (innerhalb der ersten 3 Monate nach Implantation) Herzklappen bzw. reduzierter kardialer Pumpfunktion ist aber so hoch [127], dass eine Überbrückungstherapie sinnvoll erscheint. Eine enge Abstimmung zwischen Gastroenterologen und Kardiologen sollte erfolgen. Der optimale Zeitpunkt bis zum Beginn der Überbrückungstherapie ist unklar, sinnvoll erscheint, in Abhängigkeit vom individuellen Blutungs- bzw. Thromboembolierisiko, ein Beginn innerhalb von 72 Stunden nach der Blutung.

\section{Wann sollte die Korrektur der Gerinnungssituation kontrolliert werden? Wie können/sollen DOAKs antagonisiert werden?}

\section{EMPFEHLUNG 31A}

Bei chronischem Blutverlust und klinisch stabiler Situation kann die antithrombotische Therapie bis zur Endoskopie fortgeführt werden.

Offene Empfehlung, Konsens.

\section{EMPFEHLUNG 31B}

Bei akuter Blutung oder klinisch instabiler Situation soll die Antikoagulation bis zur Notfall-Endoskopie ausgesetzt werden.

Starke Empfehlung, starker Konsens.

\section{EMPFEHLUNG 31C}

Bei schwerer gastrointestinaler Blutung kann die Antikoagulation (DOAKs, Vitamin-K-Antagonist) vor der endoskopischen Blutstillung antagonisiert werden.

Offene Empfehlung, starker Konsens.

\section{EMPFEHLUNG 31 D}

Bei der Indikationsstellung sollte das individuelle Thromboembolierisiko berücksichtigt werden.

Empfehlung, starker Konsens. 


\section{EMPFEHLUNG 32A}

Nach erfolgreicher endoskopischer Blutstillung kann die Antikoagulation (DOAKs, Vitamin-K-Antagonist) unter Berücksichtigung des individuellen Blutungsrisikos und des Thromboembolierisikos wieder fortgeführt werden. Eine Antagonisierung ist nicht erforderlich.

Empfehlung, starker Konsens.

\section{EMPFEHLUNG 32B}

Bei primär erfolgreicher endoskopischer Blutstillung soll keine Antagonisierung einer gerinnungshemmenden Therapie erfolgen.

Starke Empfehlung, starker Konsens.

\section{EMPFEHLUNG 32C}

Bei fraglicher endoskopischer Blutstillung kann eine Antagonisierung von DOAKs erwogen werden, sofern ein klinisch relevanter Hämoglobin-Abfall innerhalb der Halbwertszeit eintritt.

Offene Empfehlung, starker Konsens.

\section{EMPFEHLUNG 32 D}

Bei interventionell nicht beherrschbarer gastrointestinaler Blutung unter Antikoagulation sollte die Gerinnung korrigiert werden (z. B. Antagonisierung bei DOAKs oder Gerinnungsfaktoren bei Vitamin-K-Antagonisten).

Empfehlung, Konsens.

\section{EMPFEHLUNG 33A}

DOAKs sollen bei vital bedrohlicher Blutung mit PPSB oder aPPSB antagonisiert werden.

Starke Empfehlung, starker Konsens.

\section{EMPFEHLUNG 33B}

Sofern spezifische Inhibitoren zur Verfügung stehen, können diese eingesetzt werden.

Offene Empfehlung, starker Konsens.

\section{EMPFEHLUNG 33C}

Bei Frührezidiv der gastrointestinalen Blutung kann die Antagonisierung der Antikoagulation (DOAKs, Vitamin-K-Antagonist) erfolgen.

Offene Empfehlung, Konsens.

Der Umgang mit gerinnungsaktiven Substanzen bei elektiver Endoskopie wird ausführlich in der Leitlinie „Qualitätssicherung in der gastrointestinalen Endoskopie“ abgehandelt [109] und soll in diesem Rahmen nicht wiederholt werden. Die frühelektive Endoskopie sollte sich an den dort vorgeschlagenen Empfehlungen orientieren.

Das Absetzen von niedrig dosierter Acetylsalicylsäure, die im Rahmen einer kardiovaskulär indizierten Sekundärprophylaxe indiziert ist, erhöht das Risiko kardiovaskulärer und zerebrovaskulärer Ereignisse dreifach, wobei die Mehrzahl der Ereignisse innerhalb von 7 bis 10 Tagen auftraten $[121,128]$. In einer randomisierten und kontrollierten Studie bei Patienten mit hohem Rezidivblutungsrisiko bei gastroduodenalen Ulzera und hohem ischämischem Risiko führte das Absetzen von ASS zwar zu einem niedrigeren Rezidivblutungsrisiko (5,4 vs. 10,3\% in Woche 4), die Mortalität stieg aber in der Plazebo-Gruppe zu Woche 8 10-fach an (1,3 vs. 12,9\%) [120]. Der antithrombozytäre Effekt des ASS hielt etwa fünf Tage an, während das Risiko einer Rezidivblutung in den ersten 3 Tagen besonders hoch war. ASS sollte daher nicht, oder in Abhängigkeit vom Rezidivblutungsrisiko allenfalls für 72 Stunden pausiert werden [1].

Prinzipiell ist die endoskopische Blutstillung auch bei Patienten, die orale Antikoagulanzien einnehmen, effektiv [129]. In einer Studie am oberen Gastrointestinaltrakt, in der Varizenblutungen ausgenommen worden waren, wurden zwei Patientenkollektive bezüglich ihrer Re-Blutungsrate nach endoskopischer Blutstillung verglichen. In der ersten Gruppe waren die Patienten nicht antikoaguliert (INR <1,3). In der zweiten Gruppe waren die Patienten mit Vitamin-K-Antagonisten antikoaguliert (INR 1,3 bis 2,7). Die Re-Blutungsrate nach Blutstillung betrug 21 vs. $23 \%$ und war nicht signifikant unterschiedlich. Zusammenfassend ist bei leicht bis mittelgradig antikoagulierten Patienten die endoskopische Blutstillung effektiv und die Re-Blutungsrate im Vergleich zu nicht antikoagulierten Patienten nicht erhöht. Auch wenn von einem ähnlichen bzw. für einzelne Substanzen geringerem Risiko für schwere Blutungen unter DOAK im Vergleich zu Vitamin-K-Antagonisten ausgegangen werden kann [130], existieren aktuell keine Studien bezüglich der Erfolgsrate der endoskopischen Blutstillung und der Re-Blutungsrate nach Intervention unter DOAKs.

Bei der vital bedrohlichen Blutung steht die Blutstillung auch bei Patienten mit hohem Thromboembolierisiko im Vordergrund. Daher sollten in dieser Situation DOAKs antagonisiert werden. Prospektive Studien zur Erfolgsrate der Blutstillung nach Antagonisierung von DOAKs existieren jedoch nicht.

Als effektiv bei der Behandlung der refraktären Blutung ist die Gabe von PPSB 20-40 IU/kg Körpergewicht oder aPPSB anzusehen [131]. 
Eine Studie von Marlu et al. zeigte, dass aPPSB sowohl Dabigatran als auch Rivaroxaban am effektivsten antagonisiert, auch PPSB und rFVIIA waren wirksam [132].

Spezifische Antikörper gegen DOAKs sind Ende 2015 in der EU zugelassen worden. Idarucizumab kann als spezifisches Dabigatran-Antidot eingesetzt werden. Eine weitere Substanz, Andexanet alfa (PER977), bindet an verschiedene DOAKs (Dabigatran, Edoxaban, Apixaban, Rivaroxaban) sowie an niedrigmolekulare Heparine und Fondaparinux. In einer ersten klinischen Studie konnte gezeigt werden, dass die Wirkung von PER977 nach 2 bis 5 Minuten eintritt und den Effekt von Edoxaban $60 \mathrm{mg}$ komplett für 24 Stunden antagonisiert.

\section{Arbeitsgruppe 3: Diagnostik}

\section{EMPFEHLUNG 34}

Bei Hämatochezie mit hämodynamischer Instabilität sollte vor einer Koloskopie eine Gastroskopie zum Ausschluss einer schweren oberen gastrointestinalen Blutung erwogen werden.

Empfehlung, Starker Konsens.

\section{Obere gastrointestinale Blutung}

Eine Reihe von endoskopischen (ÖGD, Koloskopie, Ballonenteroskopie und Kapselenteroskopie) und nicht-endoskopischen Verfahren (CT-Angiografie, Angiografie, Szintigrafie) stehen zur Lokalisation der Blutungsquelle zur Verfügung. Die Methode der Wahl zur Diagnostik bei der akuten gastrointestinalen Blutung richtet sich nach der klinischen Präsentation.

Die ÖGD ist die endoskopische Diagnostik der Wahl bei Zeichen der oberen gastrointestinalen Blutung und ggf. auch bei massiver Haematochezie, um eine massive obere gastrointestinale Blutung auszuschließen.

\section{Untere gastrointestinale Blutung}

Etwa $10 \%$ der Patienten mit einer schweren Hämatochezie haben eine obere gastrointestinale Blutung [133]. In einer Studie mit 727 Patienten zeigten 14\% der Patienten mit oberer gastrointestinaler Blutung eine Hämatochezie; ein Ulkus duodeni war bei $44 \%$ der Fälle die häufigste Ursache. Eine weitere Arbeit konnte bei 13 von 85 Patienten mit einer Hämatochezie (15\%) eine obere gastrointestinale Blutung feststellen [134]. Eine mögliche Schlussfolgerung ist daher, dass bei hämodynamisch instabilen Patienten mit schwerer Hämatochezie eine Gastroskopie vor der Koloskopie durchgeführt werden sollte.

\section{EMPFEHLUNG 35}

Bei unterer gastrointestinaler Blutung mit hämodynamischer Instabilität sollte nach Ausschluss einer anorektalen Blutungsquelle eine Koloskopie innerhalb von 12 Stunden nach Aufnahme erfolgen. Dabei sollte eine verkürzte und intensivierte Darmvorbereitung durchgeführt werden.

Empfehlung, Konsens.

Insgesamt existieren nur wenige Arbeiten, die den Zeitpunkt der Untersuchung bei einer unteren gastrointestinalen Blutung definieren. Empfehlungen für die Bewertung von Patienten mit unterer gastrointestinaler Blutung basieren überwiegend auf klinischen Erfahrungen. Bislang liegen keine großen randomisierten Studien vor, die einen klaren Vorteil einer bestimmten Strategie demonstrieren. Ein wesentlicher Unterschied zur Blutung im oberen Gastrointestinaltrakt ist, dass die Endoskopie bei akuter Blutung aus dem Kolon durch Stuhl- und Blutverschmutzungen deutlich erschwert ist, zudem zeigt sich bei unvorbereitetem Kolon eine erhöhte Perforationsgefahr [135]. So wurde belegt, dass nach intensivierter Darmvorbereitung mit 5-6L Abführlösung innerhalb von 3 Stunden aktive Blutungen endoskopisch sicherer identifiziert werden und so eine Hämostase erreicht werden kann $[136,137]$. Die Notfallkoloskopie wird als Untersuchung definiert, die 12 - 24 Stunden nach der Aufnahme des Patienten erfolgt [138]. So konnte gezeigt werden, dass die Identifikation der Blutungsquelle im unteren Gastrointestinaltrakt bei Patienten mit Hämatochezie und Divertikeln bei $42 \%$ lag, wenn die Untersuchung innerhalb von 12 Stunden nach Klinikaufnahme erfolgte. Im Gegensatz dazu lag die Detektionsrate bei $22 \%$, wenn die Untersuchung erst nach 27 - 72 Stunden erfolgte [139]. Eine andere kontrollierte randomisierte Studie zeigte ähnliche Ergebnisse ( 6 vs. $0 \%$ ), allerdings ohne statistisch signifikanten Unterschied zwischen Notfall- und elektiver Koloskopie [134]. So wird von der Amerikanischen Gesellschaft für gastrointestinale Endoskopie bei Patienten mit schwerer Hämatochezie eine Koloskopie innerhalb von 24 Stunden mit einer schnellen Darmvorbereitung empfohlen [21]. Mit zunehmendem Abstand vom Zeitpunkt der Aufnahme des Patienten sinkt die erfolgreiche endoskopische Therapie. Sofern die Koloskopie in den ersten 12 Stunden nach Aufnahme erfolgt, zeigen sich Erfolge in $29 \%$ der Untersuchungsfälle gegenüber $0 \%$, wenn die Untersuchung erst nach 48 Stunden erfolgt. In der Bewertung dieser Daten ist die hohe Rate an spontan sistierenden Blutungen zu bedenken. Niikura und Kollegen konnten neben einer frühen Notfallkoloskopie weitere Prädiktoren zur Identifikation einer Blutung im unteren Gastrointestinaltrakt finden. So hatten die Erfahrung des Untersuchers, eine Kappe auf der Endoskopspitze und der Einsatz eines Endowashers einen signifikant positiven Einfluss auf die Blutungsdetektion [140]. Wenngleich ein spontaner Blutungsstillstand sehr häufig ist, zeigt die endoskopische Diagnostik innerhalb der ersten 24 Stunden sowohl einen diagnostischen als auch einen therapeutischen Wert [136, 141].

In beiden o. g. Fällen sollte die Untersuchung in einer Umgebung stattfinden, in der ausreichende Überwachungsoptionen 


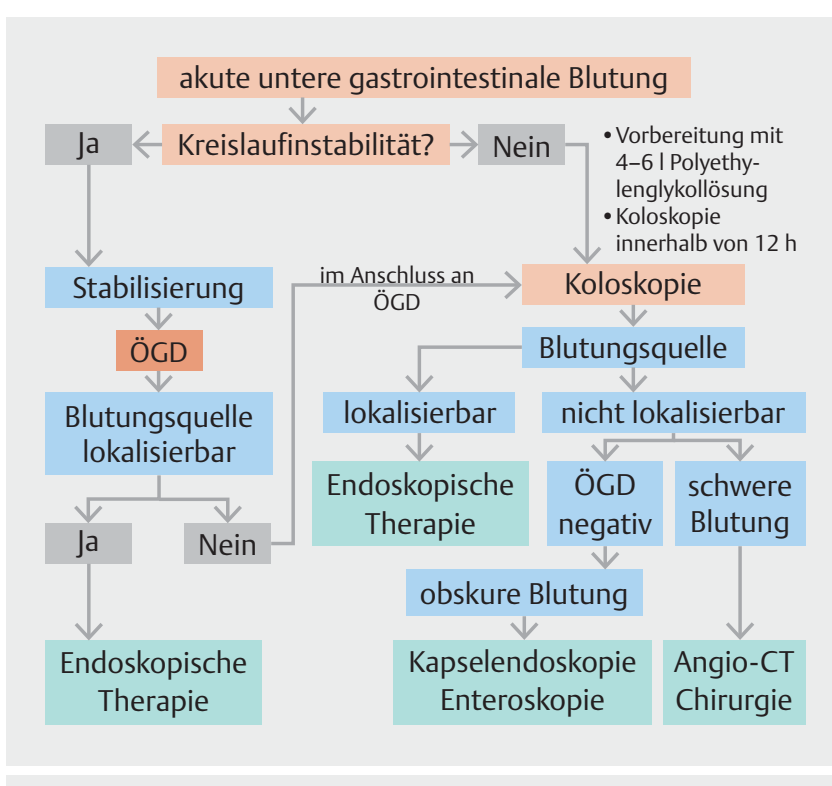

- Abb. 2 Differenzierter Einsatz der diagnostischen Optionen bei unterer gastrointestinaler Blutung [36].

und auch ausreichend ausgebildetes Personal vorhanden sind. Dies hängt sehr von der jeweiligen Infrastruktur der Klinik ab. Weitere Details sind der Leitlinie „Sedierung in der gastrointestinalen Endoskopie“ zu entnehmen [40] (

\section{EMPFEHLUNG 36}

Bei Patienten mit Indikation zur stationären Versorgung einer gastrointestinalen Blutung soll die Behandlung in einer Einrichtung erfolgen, in der binnen 12 Stunden eine Gastroskopie bzw. eine Koloskopie durchgeführt werden kann.

Starke Empfehlung, starker Konsens.

Das initiale Management von Patienten mit Zeichen einer gastrointestinalen Blutung beinhaltet noch vor der endoskopischen Diagnostik und Therapie die Einstufung der Gefährdung, die Sicherung der Vitalfunktionen und ggfs. die Stabilisierung des Kreislaufs mittels Volumen- und Blutproduktegaben [46, 142]. Zusätzlich sollte in speziellen Situationen (Hämatemesis, Enzephalopathie und unkooperativen Patienten) eine endotracheale Intubation zum Schutz der Atemwege vor Aspiration möglich sein [1]. Diese Maßnahmen sind im Regelfall in adäquatem Zeitrahmen in Akutkrankenhäusern mit internistisch oder anästhesiologisch betreuten Intensivstationen durchführbar.

Für obere gastrointestinale Blutungen insgesamt zeigt sich kein eindeutiger Überlebens-Vorteil für eine frühzeitige Endoskopie (binnen 6 bzw. 12 h), jedoch ließen sich geringere Kosten (u. a. durch geringere Transfusionsnotwendigkeiten) und eine höhere Rate an ambulant weiterzuführenden Patienten zeigen, wenn eine Gastroskopie binnen 12 - 24 h durchgeführt wurde [22, 143, 144]. Eine retrospektive Analyse zeigte eine mehr als 24-stündige Dauer bis zur ersten Endoskopie als unabhängigen Risikofaktor für blutungsassoziierte Letalität bei Patienten mit nicht-variköser oberer gastrointestinaler Blutung [145]. In Berücksichtigung dieser Umstände sollte die Versorgung von Patienten mit Verdacht auf gastrointestinale Blutung in einer Einrichtung stattfinden, die eine endoskopische Evaluation und Therapie in diesem Zeitrahmen gewährleisten kann.

\section{EMPFEHLUNG 37}

Bei Kreislaufinsuffizienz soll die endoskopische Diagnostik bzw. Therapie unter intensivmedizinischen Bedingungen nach bzw. unter Stabilisierung des Patienten erfolgen. Starke Empfehlung, starker Konsens.

\section{EMPFEHLUNG 38}

Bei Verdacht auf variköse obere gastrointestinale Blutung sollte ebenfalls eine intensivmedizinische Stabilisierung des Patienten im Vordergrund stehen, danach sollte baldmöglichst eine Endoskopie zur Diagnostik und ggfs. Therapie angeschlossen werden.

Empfehlung, starker Konsens.

Angesichts der möglichen Notwendigkeit einer intensivmedizinischen Versorgung bzw. der raschen Atemwegssicherung bei Aspirationsgefahr kann die Endoskopie auch auf einer IntermediateCare- bzw. Intensivstation stattfinden.

Unabhängig vom Ort der endoskopischen Intervention zeigen Patienten mit endoskopischer Notfallintervention bei oberer gastrointestinaler Blutung und höherer ASA-Klasse ein erhöhtes Morbiditäts- und Letalitätsrisiko im Rahmen der Sedierung [39].

\section{AUSSAGE 4}

Ausreichende personelle Voraussetzungen zur endoskopischen Diagnostik bzw. Therapie umfassen neben dem ärztlichen Untersucher und der Assistenz mindestens eine weitere ausreichend ausgebildete Person, die die Vitalfunktionen des Patienten überwacht bzw. sichert und die Sedierung bzw. Narkose führt.

Konsens.

Zur Sicherung und Überwachung der Vitalfunktionen und der prozedurbezogenen Sedierung wird daher der Einsatz einer nur mit diesen Aufgaben betrauten ausreichend ausgebildeten Person gefordert, hierzu wird auf die Leitlinien „Qualitätsanforderungen in der gastrointestinalen Endoskopie“ (AWMF-Registernummer 021 - 022) und „Sedierung in der gastrointestinalen Endoskopie“ (AWMF-Nummer 021 - 14) sowie auf die Sondersituation einer Notfallendoskopie verwiesen $[2,40]$. 


\section{AUSSAGE 5}

Propofol scheint als Sedativum zur Notfallendoskopie bei gastrointestinaler Blutung sicher zu sein.

Starker Konsens.

Zu den besonderen Umständen einer Notfallendoskopie verweisen wir auf die aktuelle S3-Leitlinie zur Sedierung in der gastrointestinalen Endoskopie [40]. In Kapitel 1.4. „Empfehlung Risikoabschätzung “ weist die Stellungnahme darauf hin, dass Notfallendoskopien zu einer Hochrisikosituation gehören und daher in diesen Fällen ein zweiter Arzt für die Durchführung und Überwachung der Sedierung empfohlen wird (kein NAPS). Hinsichtlich der Sedierung des Patienten bedarf es einer individuellen Abschätzung des Patientenzustandes (ASA-Klassifikation) und der Schwere der Blutung.

Hinsichtlich der Verwendung einer bestimmten Substanz zur Sedierung im Rahmen einer Notfallendoskopie bei oberer gastrointestinaler Blutung existieren kaum Daten. Im Hinblick auf die Verwendung von Propofol bei nicht-intubierten Patienten konnte in einer Studie von Tohda gezeigt werden, dass die Rate an Hypotonie (systolischer Blutdruck $<90 \mathrm{mmHg}$ ) und Hypoxämie (periphere Sauerstoffsättigung < 90 \%) mit 8,3 und 6,7\% höher lag als in der Kontrollgruppe (Patienten ohne obere gastrointestinale Blutung im Studienzeitraum) [146]. Bei keinem der Patienten wurde eine Maskenbeatmung oder endotracheale Intubation notwendig. Zwei Patienten entwickelten eine Pneumonie am ehesten durch Aspiration im Rahmen der Endoskopie. Der Anteil an Varizenblutungen wurde von den Autoren selbst als sehr niedrig angegeben und mit dem guten Outcome der Patienten assoziiert.

In einer weiteren allerdings retrospektiven Studie von Park et al. wurde das Outcome für die Verwendung von Propofol im Rahmen der OGIB bei Patienten mit nicht-varikösen und varikösen Blutungen verglichen. Von 703 Endoskopien wurden 539 bei nicht-varikösen und 164 bei varikösen Blutungen durchgeführt. Schock (definiert als Abfall des mittleren arteriellen Blutdruckes unter $60 \mathrm{mmHg}$ ) trat gehäuft bei varikösen Blutungen auf $(12,2$ vs. 3,5\% nicht-variköse Blutungen, $p<0,001)$. Alle bis auf einen Patienten konnten durch intravenöse Flüssigkeitsgabe stabilisiert und die Notfallendoskopie in den meisten Fällen ohne Unterbrechung durchgeführt werden. Es zeigte sich kein Unterschied im Auftreten von Hypoxämie und paradoxen Reaktionen in Abhängigkeit der Blutungsart (nicht-variköse vs. variköse Blutung: Hypoxämie, 3,5 vs. 1,8\%, $\mathrm{p}=0,275$; paradoxe Reaktionen, 4, 1 vs. $5,5 \%, p=0,442$ ) [147].

Es liegen aktuell keine randomisierten Vergleichsstudien zur Anwendung verschiedener Sedierungsregimes vor, sodass die Verwendung des Sedativums zur Sedierung im Rahmen der OGIB immer eine individuelle Entscheidung anhand der o.g. Kriterien ist. Eine generelle Empfehlung kann daher derzeit nicht gemacht werden.

\section{EMPFEHLUNG 39}

Beim Patienten mit Verdacht auf aktive gastrointestinale Blutung ohne Identifizierung der Blutungsquelle durch endoskopische Diagnostik sollte eine kontrastverstärkte MehrzeilenComputertomografie (CT) durchgeführt werden. Empfehlung, starker Konsens.

Die CT hat in den 90er Jahren durch die Entwicklung der Spiral-CT und im Folgenden der Mehrzeilenspiral-CT erhebliche Fortschritte gemacht. Die Möglichkeit, das gesamte Abdomen in mehreren spezifischen Kontrastmittelphasen einschl. der sog. CT-Angiografie untersuchen zu können, erlaubt eine nichtinvasive Diagnostik von akuten oberen und unteren gastrointestinalen Blutungen [148-151].

Für den primären Einsatz der CT spricht die sehr gute örtliche wie zeitliche Verfügbarkeit und die hohe Untersuchungsgeschwindigkeit. Aus diesen Gründen spielen MRT und nuklearmedizinische Verfahren bei der akuten GI-Blutung keine relevante Rolle.

Aus experimentellen Studien ist bekannt, dass gastrointestinale Blutungen bereits ab einer Blutungsrate von $0,5 \mathrm{ml} / \mathrm{min}$ mittels CT diagnostiziert werden können [152], in einer neueren Studie unter Verwendung eines 64-Zeilen CTs bereits ab $0,25 \mathrm{ml} / \mathrm{min}$ [153].

In einer frühen Metaanalyse betrug die Sensitivität der CT in der Diagnostik der akuten Gl-Blutung $86 \%$ bei einer Spezifität von $95 \%$ [154]. Eine andere Metaanalyse errechnete ähnliche Werte für Sensitivität (89\%) und Spezifität (85\%) [155]. Die Autoren folgern, dass die CT-Angiografie eine akkurate, kosteneffektive Methode zur Diagnostik der akuten gastrointestinalen Blutung sei, die genaue Lokalisation darstellen könne und somit über das weitere therapeutische Vorgehen entscheiden helfe.

In einer europäischen systematischen Metaanalyse von 2013 konnten 22 Studien ausgewertet werden (672 Patienten, mittleres Alter 65) [156]. Die Gesamtsensitivität der CT-Angiografie in der Detektion einer aktiven gastrointestinalen Blutung wird mit $85,2 \%$ (95\% KI 75,5\% bis 91,5\%) angegeben, die Spezifität mit $92,1 \%$ (95\% KI 76,7\% bis $97,7 \%)$.

Neben der reinen Lokalisationsdiagnostik kann die CT Informationen zur Blutungsursache bieten und helfen, eine Therapieentscheidung (endoskopisch, radiologisch-interventionell, chirurgisch) zu fällen.

\section{EMPFEHLUNG 40}

Die CT zur Detektion einer akuten gastrointestinalen Blutung soll als Mehrzeilenspiral-CT in mehrphasiger Technik durchgeführt werden, wobei mindestens eine arterielle Phase nach intravenöser Kontrastmittelgabe akquiriert werden sollte. Eine positive enterale Kontrastierung soll vor der CT nicht erfolgen.

Starke Empfehlung, starker Konsens. 
In der Regel werden dreiphasige Protokolle (nativ, arteriell, venös) empfohlen [157, 158], wobei experimentell die Kombination aus arterieller und venöser Phase die höchste Sensitivität aufwies [153]. Eine positive enterale Kontrastierung kann die Extravasation des intravenös applizierten KMs maskieren und zu falsch negativen Befunden führen [158, 159]. Eine negative oder neutrale Kontrastierung des GI-Traktes kann hilfreich sein, soll aber bei entsprechendem Zeitdruck die initiale CT-Diagnostik nicht verzögern.

\section{EMPFEHLUNG 41}

Wenn die CT in adäquater Technik keine Blutungsquelle zeigt, sollte eine selektive Angiografie nicht durchgeführt werden.

Die CT sollte so früh wie möglich nach Blutungsbeginn bzw. Endoskopie ohne Blutungsquellennachweis erfolgen. Sie kann ggfs. wiederholt werden.

Empfehlung, Konsens.

Wenn die CT in adäquater Technik keine Blutungsquelle zeigt, ist auch eine anschließende Angiografie in der Regel unauffällig. In einer Single-Center-Serie aus Cleveland mit 86 CT-Angiografien bei 74 Patienten über einen Zeitraum von 4,5 Jahren konnte in keinem Fall nach initial negativem CT in einer Folgeangiografie innerhalb von $24 \mathrm{~h}$ eine Blutungsquelle identifiziert werden [160]. Eine Wiederholung der CT-Angiografie im Intervall kann dagegen die Blutung zeigen. Weil die CT wie alle anderen Verfahren darauf angewiesen ist, dass zum Zeitpunkt der Untersuchung eine aktive Blutung vorliegt, sollte eine Untersuchung so schnell wie möglich nach Symptombeginn angestrebt werden [158].

\section{AUSSAGE 6}

Als chronische Blutung des Gastrointestinaltrakts werden anhaltende Blutungen mit Abfall des Serumhämoglobinspiegels bezeichnet, die nicht zur Kreislaufinstabilität führen und mit Eisenmangel einhergehen.

Starker Konsens.

Der Patient mit chronischer Blutung kann symptomatisch, aber auch asymptomatisch sein, da oft eine gute Adaption besteht; bei der adaptierten Anämie handelt es sich oft um einen Zufallsbefund. Eine fundierte Anamnese deckt häufig Symptome auf, die der Anämie nicht zugeordnet oder negiert wurden. Die Art der Anämie muss differenziert werden, um die Weichen für die Diagnostik zu stellen. Die eindeutige Differenzierung der Eisenmangelanämie führt zu einer entsprechenden endoskopischen Diagnostik. Andere mögliche Blutungsquellen (z. B. Hypermenorrhö oder rezidivierende Epistaxis) sollten vorab ausgeschlossen werden. Zur Differenzierung der Anämie sollten weitere Laborparameter herangezogen werden (großes Blutbild, Serum-Ferritin, Serum-Transferrinsättigung). Die Eisenmangelanämie ist eine mikrozytäre, hypochrome Anämie mit Unterschreitung der geschlechtsspezifischen Normwerte des Serumhämoglobinspiegels
(Hb) sowie Verringerung des Serum-Ferritin, der Transferrinsättigung oder des Serum-Eisen (nach Abstufung hoher zu niedriger Relevanz in dieser Reihenfolge). Die Parameter Ferritin und Transferrinsättigung können gerade in Grenzbereichen des Serumhämoglobingehalts zur Sicherheit der Einstufung der Anämie beitragen. Die gastrointestinale Blutung kann sowohl offensichtlich als auch okkult (nicht sichtbar und nur durch spezifische Stuhltests nachweisbar) auftreten. Ein fehlendes klinisches Blutungszeichen schließt die gastrointestinale Blutung also nicht aus. Die genaue Differenzierung ist auch im Hinblick auf die Kostenerstattung wichtig (Kapselendoskopie).

\section{EMPFEHLUNG 42}

Die chronische gastrointestinale Blutung kann ambulant abgeklärt werden.

Offene Empfehlung, starker Konsens.

Aufgrund der Eigenheit der chronischen gastrointestinalen Blutung (stabiler Patient) ist eine ambulante Abklärung sinnvoll und kosteneffektiv. Lassen die klinischen Zeichen eine Zuordnung der Blutungsquelle zum oberen oder unteren Gastrointestinaltrakt zu, sollte die endoskopische Diagnostik adaptiert erfolgen (primär Gastroskopie bei klinischen Zeichen der oberen, primär Koloskopie bei Zeichen der unteren gastrointestinalen Blutung). Hierdurch kann die Diagnostik beschleunigt werden. Lässt sich die Blutung nicht eindeutig zuordnen (z. B. bei der okkulten Blutung), sollte nach statistischer Häufigkeit der Blutungsquelle zunächst die ÖGD und dann ggf. die lleokoloskopie durchgeführt werden [161].

\section{AUSSAGE 7}

Eine frühzeitige endoskopische Diagnostik ist kosteneffektiv. Konsens.

Die zeitnahe Durchführung der Diagnostik erhöht die Ausbeute in Hinsicht auf die Diagnosestellung. Je länger das Intervall zwischen dem potenziellen Blutungsereignis (overte oder okkulte Blutung und/oder HB-Abfall) ist, desto geringer ist die diagnostische Ausbeute. Bei früher Diagnosestellung werden Folgekosten durch Therapie oder wiederholter Diagnostik gesenkt [144, 162, 163].

\section{EMPFEHLUNG 43}

Eine wiederholte endoskopische Diagnostik sollte bei unzureichender Qualität der Indexendoskopie und bei erhöhtem Risiko für familiär gehäuftes kolorektales Karzinom erfolgen. Empfehlung, Konsens. 


\section{EMPFEHLUNG 44}

Besteht weiterhin der Verdacht auf eine aktive obere oder untere gastrointestinale Blutung, kann eine ÖGD oder Ileokoloskopie wiederholt werden.

Offene Empfehlung, Konsens.

Eine Wiederholung der Endoskopie kann in bestimmten Fällen die Diagnoserate erhöhen: Bei eingeschränkter Sicht oder hoher familiärer Belastung bzgl. kolorektalen Karzinoms sollte die endoskopische Diagnostik wiederholt werden, da hierdurch die diagnostische Ausbeute in dieser Entität gesteigert wird [164, 165].

Eine Wiederholung der qualitativ einwandfreien bidirektionalen Endoskopie steigert nicht die diagnostische Ausbeute, sondern führt zur Verzögerung der Diagnosestellung, da das Intervall zwischen dem potenziellen Blutungsereignis und den durchzuführenden weiterführenden Untersuchungen verlängert wird [166].

\section{AUSSAGE 8}

Zum Blutungsprovokationsmanöver durch Gabe von Antikoagulanzien kann aufgrund der aktuellen Datenlage keine Empfehlung abgegeben werden.

Starker Konsens.

Die Datenlage zu Provokationsmanövern per Antikoagulation ist uneinheitlich. In einer Serie war durch die Provokation einer Blutung zur Verbesserung der Lokalisation mittels Antikoagulanzien keine Steigerung der Diagnoserate zu erreichen. Die Komplikationsrate wird jedoch erhöht [167]. Eine aktuelle retrospektive Serie aus Lousiana analysierte eine Datenbank zur Devices-assistierten Enteroskopie bei mittlerer GI-Blutung. Insgesamt konnten 824 Prozeduren analysiert werden. 38-mal erfolgte bei insgesamt 27 Patienten eine medikamentöse Provokation. In der Gruppe, die noch nie Antikoagulanzien eingenommen hatte, erfolgte diese Provokation mit Clopidogrel und Heparin und bei 71 \% (5/7) konnte ein therapierbarer pathologischer Befund gefunden werden. Unerwünschte Wirkungen traten in dieser Gruppe nicht auf. Der diagnostische Ertrag der Provokation in der Gruppe, die bereits zuvor Antikoagulanzien einnahm, lag demgegenüber bei $53 \%$ [168].

\section{EMPFEHLUNG 45}

Führt die bidirektionale endoskopische Diagnostik (ÖGD und Ileokoloskopie) bei chronischer gastrointestinaler Blutung nicht zu einem Blutungsquellennachweis, sollte zeitnah eine Kapselendoskopie des Dünndarms (KE) durchgeführt werden. Empfehlung, Konsens.

Die KE ist als nicht-invasives Verfahren geeignet, eine Blutungsquelle des mittleren GI-Traktes zu detektieren. Die Sensitivität und Spezifität der Untersuchung sind hoch [161, 162, 169]. Die Wahrscheinlichkeit, die Blutungsquelle zu finden, ist höher, wenn die Diagnostik zeitnah erfolgt, optimal ist ein Zeitfenster von 14 Tagen [166]. Positive prädiktive Faktoren für die Feststellung eines pathologischen Dünndarmbefunds sind die overte Blutung, männliches Geschlecht und ein Alter > 60 Jahre [170].

\section{EMPFEHLUNG 46}

Eine Kapselendoskopie soll bei bekannter hochgradiger Stenose des Gastrointestinaltrakts nicht durchgeführt werden. Bei klinischem Verdacht auf eine intestinale Stenose soll die Kapselendoskopie erst nach Beweis der Durchgängigkeit mittels vorheriger Patency-Kapseluntersuchung erfolgen.

Starke Empfehlung, Konsens.

Die bekannte hochgradige Dünndarmstenose stellt eine Kontraindikation zur KE dar. Die Anamnese ist in der Regel richtungsweisend [171, 172]. Die Kapselretention (Kapselpersistenz im Dünndarm > 3 Tage) stellt per se keinen Notfall dar, wenn keine lleussymptomatik vorliegt, sondern zeigt durch die Kapsellage die zu diagnostizierende Stenose an, die einer entsprechenden Therapie zugeführt werden kann. Ein akuter Ileus als Folge einer Kapselendoskopie ist selten [172].

Andere bildgebende Verfahren (z. B. MRT) schließen eine Stenose nicht sicher aus. Die Pilotkapsel (Patency-Kapsel) kann zum Nachweis einer erwartbar komplikationslosen Kapselpassage herangezogen werden, auch wenn die Passage der Dünndarmkapsel damit nicht gesichert ist, sondern nur wahrscheinlicher wird $[173,174]$.

Bei negativer Anamnese ist ein genereller Ausschluss einer Dünndarmstenose mittels Patency-Kapsel oder bildgebender Diagnostik vor einer KE mangels Effektivität und Steigerung der Kosten nicht empfohlen [166].

\section{EMPFEHLUNG 47}

Bei Patienten unter Antikoagulanzientherapie mit persistierender Anämie sollte die endoskopische Diagnostik wiederholt werden.

Empfehlung, Konsens.

Ein unauffälliger Befund in der KE bei vorangegangener Endoskopie hat einen hohen negativen prädiktiven Wert bzgl. des Vorliegens einer Blutungsquelle. Eine Rezidivblutung ist bei diesen Patienten seltener, davon ausgenommen sind Patienten unter Antikoagulanzientherapie. Hier ist der negative prädiktive Wert für das Vorliegen einer möglichen Blutungsquelle im Dünndarm deutlich niedriger $[175,176]$. 


\section{EMPFEHLUNG 48}

Zur endoskopischen Diagnostik einer Blutung im mittleren Gastrointestinaltrakt soll die Device-assistierte Enteroskopie (DAE) angewandt werden.

Starke Empfehlung, Konsens.

Die Push-Enteroskopie kann bei proximalen Läsionen verwendet werden.

Offene Empfehlung, Konsens.

Die Device-assistierte Enteroskopie (DAE) (hier liegen die meisten Daten für die Doppelballonenteroskopie [DBE] vor) hat eine hohe Sensitivität und Spezifität zur Detektion der Blutungsquelle bei der mittleren gastrointestinalen Blutung. Im Vergleich dazu ist die KE ebenbürtig, weist aber eine wesentlich geringere Komplikationsrate auf. Die DAE kann in der first-line-Diagnostik nicht empfohlen werden, sondern folgt als therapeutisches Instrument der KE. Eine Ausnahme stellen hier die Patienten mit einer laufenden Antikoagulanzientherapie dar, bei denen die DAE wegen des oben beschriebenen schlechteren negativen prädiktiven Wertes für das Vorliegen einer Blutungsquelle eine höhere diagnostische Trefferquote erreicht $[175,176]$.

\section{EMPFEHLUNG 49}

Die intraoperative Enteroskopie, Angiografie oder Technetium-Szintigrafie sind Reserveverfahren zur Lokalisationsdiagnostik einer okkulten gastrointestinalen Blutung und sollten auf Einzelfälle beschränkt werden.

Empfehlung, starker Konsens.

Im Vergleich zur intraoperativen Enteroskopie hat die KE eine höhere Sensitivität und Spezifität [173]. Eine diagnostische intraoperative Enteroskopie kann wegen der Invasivität nicht empfohlen werden. Die radiologischen Verfahren haben bei der Abklärung der chronischen GIB methodenbedingt eine schlechtere Sensitivität $[154,176]$.

\section{AUSSAGE 9}

Relevante postoperative gastrointestinale Blutungen sind selten. Meist ist nicht das Operationstrauma, sondern eine exazerbierte vorbestehende Blutungsquelle bzw. ein nicht direkt-OP-bezogener Faktor ursächlich.

Starker Konsens.

Es gibt wenig publizierte Evidenz zum Thema postoperative gastrointestinale Blutungen. Viele gastrointestinale Blutungen sind selbstlimitierend, und relevante post-OP Blutungen (definiert als Blutungen mit hämodynamischer Auswirkung, Verlust von $2 \mathrm{~g} / \mathrm{dl}$ Hämoglobin, Notwendigkeit einer Transfusion oder Notwendigkeit einer Intervention) sind selten.
- Tab. 6 Chirurgische Eingriffe und postoperative gastrointestinale Blutungsinzidenzen.

\begin{tabular}{|l|l|l|}
\hline Operation & Inzidenz & Ref. \\
\hline $\begin{array}{l}\text { Gastrointestinale Anastomosen } \\
\text { bei Magen-NPL }\end{array}$ & $0,4 \%$ & {$[177]$} \\
\hline $\begin{array}{l}\text { Gl-Anastomosen im Rahmen } \\
\text { bariatrischer OPs }\end{array}$ & $0,4-1 \%$ & {$[178]$} \\
\hline $\begin{array}{l}\text { Kolon-Anastomosen } \\
\text { (Hand/Stapler) }\end{array}$ & $0,6-4 \%$ & {$[179-182]$} \\
\hline \begin{tabular}{l} 
Hepatobiliopankreatische Chirurgie \\
\hline Herzchirurgie, Aortenbogenchirurgie
\end{tabular} & $2-5 \%$ & {$[183-185]$} \\
\hline \begin{tabular}{l} 
Gefäßchirurgische aortale Eingriffe \\
\hline Multimorbide Patienten (ünabhängig \\
vom Eingriff)
\end{tabular} & $0,4-2 \%$ & {$[186,187]$} \\
\hline
\end{tabular}

Postoperative gastrointestinale Blutungen entstehen üblicherweise in drei Szenarien:

a) Die Operation (oder deren Komplikationen) sind die Ursache der Blutung.

b) Die Blutung entsteht durch Ursachen, die nicht direkt operationsbezogen sind (z. B. Überdosierung von Antikoagulanzien).

c) Der Operationsstress exazerbiert eine vorbestehende Blutungsquelle (z. B. Ulkus oder Gastritis) ( Tab. 6)

\section{Arbeitsgruppe 4: Therapie nicht-variköser Blutung}

\section{EMPFEHLUNG 50}

Blutungen aus Ulzera sollen entsprechend der Forrestklassifikation zugeordnet werden. Auch für andere nicht-variköse Blutungen kann diese in modifizierter Form verwendet werden. Hierdurch soll zwischen Ulzera mit hohem und niedrigem Risiko für andauernde bzw. rezidivierende Blutungen unterschieden werden.

Starke Empfehlung, starker Konsens.

Die Forrestklassifikation basiert auf der Arbeit John A.H. Forrest et al. aus dem Jahr 1974 und dient der standardisierten Beschreibung von Blutungen aus peptischen Ulzera [193]. Die prognostische Bedeutung dieser Einteilung unterstreichen nachfolgende Studien, die insbesondere eine Korrelation zwischen Blutungen der Stadien Fla/Flb und der Blutungspersistenz bzw. dem Auftreten von Blutungsrezidiven identifizierten [194, 195]. Das Erkennen und die richtige Zuordnung der Blutungsstigmata sind jedoch untersucher- und erfahrungsabhängig. Dies gilt insbesondere für die Stadien FII und FIII [196 - 198]. Zusätzlich zu den Kriterien der Forrestklassifikation sind die Größe der Ulzera $(>2 \mathrm{~cm})$, sowie deren Lokalisation (Hinterwand des Bulbus duodeni; proximaler Magen/kleine Kurvatur) Indikatoren eines erhöhten Rezidivblu- 
tungsrisikos. Für Blutungen aus anderen nicht-varikösen Quellen existieren keine spezifischen Klassifikationen, sodass hier die Forrestklassifikation zur Anwendung kommen kann.

\section{EMPFEHLUNG 51}

Ulzera mit arteriell spritzenden Blutungen (Fla), Sickerblutungen (Flb) oder mit sichtbarem Gefäßstumpf (FIla) sollen endoskopisch therapiert werden, da diese unbehandelt ein hohes Risiko für ein Fortbestehen bzw. erneutes Auftreten von Blutungen haben.

Starke Empfehlung, starker Konsens.

Die Effizienz der Endotherapie in den Stadien Fla, Flb und Flla hinsichtlich der Blutungspersistenz sowie Rezidivblutungsrate wurde in mehreren, prospektiv randomisierten Studien untersucht. Eine Metaanalyse dieser Arbeiten zeigte, dass die endoskopische Hämostase sowohl das Andauern von Ulkusblutungen als auch deren Rezidive reduziert. Dies gilt sowohl für aktive Blutungen (Fla, Flb: RR 0,29, $95 \%$ KI 0,20-0,43; NNT 2, $95 \%$ KI 2-2) als auch für Ulzera mit sichtbaren Gefäßstümpfen (FIla: RR 0,49, 95 \% KI 0,400,59; NNT 5, 95\% KI 4-6) [199].

\section{EMPFEHLUNG 52}

Adhärente Koagel (FIIb) sollten, wenn leicht möglich, entfernt werden, um darunterliegende aktive Blutungen bzw. Gefäßstümpfe zu identifizieren und endoskopisch gezielt zu therapieren. Patienten mit Ulzerationen der Stadien Fllc oder FIII benötigen keine spezifische endoskopische Therapie.

Empfehlung, starker Konsens.

Die Daten zur endoskopischen Blutstillung von peptischen Ulzera im Stadium FIlb sind widersprüchlich, sodass eine Empfehlung zur generellen Entfernung von Koageln nicht gerechtfertigt erscheint. Bei $25 \%$ der Patienten, die in diesem Stadium keine endoskopische Therapie erhalten, ist innerhalb von 30 Tagen mit einem Blutungsrezidiv zu rechnen [200]. Der Vergleich der alleinigen medikamentösen Therapie mit endoskopischer Hämostase reduziert dieses Risiko in einigen randomisierten, kontrollierten Studien und Metaanalysen signifikant ( 8,2 vs. $24,7 \%, p<0,01)$ [201 203]. Andererseits ergab eine Metaanalyse, die alleinig randomisierte, kontrollierte Studien auswertete, keinen signifikanten Vorteil für diese Patienten (RR 0,31, 95\% KI 0,06-1,77) [199].

Patient mit Ulcera der Stadien Fllc und FIII erleiden nur selten (Rezidiv-)Blutungen, sodass eine prophylaktische endoskopische Therapie keinen substanziellen Vorteil bietet [1].

\section{EMPFEHLUNG 53}

Zur endoskopischen Stillung nicht-variköser Blutungen können die Injektionstherapie, mechanische Verschlüsse (Hämoclips) sowie thermische Verfahren verwendet werden. Offene Empfehlung, starker Konsens.

\section{EMPFEHLUNG 54}

Die Injektionstherapie soll in der Regel mit einem zweiten, mechanischen oder thermischen Verfahren kombiniert werden, um das Rezidivblutungsrisiko zu minimieren.

Starke Empfehlung, starker Konsens.

\section{EMPFEHLUNG 55}

Für Blutungen die unter Anwendung der Standardverfahren nicht sistieren, können „over-the-scope clips“ oder Hämostasesprays genutzt werden.

Offene Empfehlung, starker Konsens.

\section{Injektionstherapie}

Die Blutstillung durch Injektion resultiert aus der lokalen Gefäßkompression infolge des Gewebeödems. Ein zusätzlicher vasokonstriktiver Effekt wird durch die Verwendung von Epinephrin, in einem Verdünnungsverhältnis von 1:10 000 bzw. 1:20 000 erreicht. Fibrinkleber, der ebenfalls per Injektion injiziert wird, verschließt blutende Gefäße und initiiert die Wundheilung [204]. Aktuelle Daten zum Vergleich mit anderen Hämostaseverfahren liegen nicht vor. Unter Berücksichtigung der Kosten und der erhöhten Anforderungen an die Applikationstechnik sollte die Fibrinkleberinjektion als Reserveverfahren angesehen werden.

\section{Thermische Verfahren}

Thermische Methoden ermöglichen einen gezielten, direkten Gefäßverschluss. Hierzu stehen verschiedene Sonden zur Verfügung. So kann durch bipolare und Heatersonden zusätzlich zur kurzzeitigen Kompression des blutenden Gefäßes ein „Verschweißen“ der Gefäßwände mit dem Ziel der dauerhaften Blutungsstase erreicht werden. Blutungen, die während endoskopischer Resektionen auftreten, können umgehend, z. B. durch ESD-Messer oder geeignete Zangen, mittels elektrischer Koagulation gestillt werden. Monopolare Sonden erreichen die Hämostase durch Fulguration, bei der Gefäße durch Funkenentladung der dicht über dem Gewebe geführten Sonde verödet werden. Ebenfalls ohne direkten Kontakt zwischen Sonde und Gewebe wird die ArgonPlasma-Koagulation angewendet. Hierbei wird nach Ionisation von Argongas zu Argonplasma Hochfrequenzstrom ohne Gewebeberührung weitergeleitet und so die Hämostase ermöglicht. 


\section{Mechanische Therapie}

Metallclips führen zur Blutstillung durch mechanischen Gefäßverschluss bzw. durch Adaptation von Wund- und Resektionsrändern. Sollte die Größe und/oder Beschaffenheit des Blutungsareals, insbesondere bei Ulkusblutungen keinen Einsatz „konventioneller" Metallclips erlauben, können durch geschulte Anwender "over-the-scope clips“ (OTSC) verwendet werden. Hierbei wird nach Ansaugen oder Hineinziehen des Gewebes in die Aufsatzkappe der OTSC über einen Fadenzug freigesetzt und so ein tiefgreifendes Erfassen der Wandschichten ermöglicht. Daten einer Studie anhand eines ex-vivo Modells zeigen, dass OTSC im Vergleich zu Standardhämoclips eine schnellere und effektivere Blutstillung gestatteten [205]. Fallserien beschreiben darüber hinaus die erfolgreiche Blutstillung durch OTSC nach frustraner endoskopischer Vortherapie bzw. auch als „first line“-Behandlung von Hochrisikoblutungen [206-208].

\section{Kombination der Verfahren}

Da die Wirkung der Injektionstherapie, insbesondere wenn Kochsalzlösung und Epinephrin verwendet werden, zeitlich begrenzt ist und somit ein erhöhtes Rezidivblutungsrisiko besteht, sollte ein weiteres, mechanisches oder thermisches Verfahren zur Anwendung kommen. Diese Empfehlung beruht auf einer Reihe randomisierter kontrollierter Studien zur Rezidivblutungsrate aus peptischen Ulzera mit Hochrisikoblutungsstigma [199, 209 212].

\section{Hämostasesprays}

Hämostasesprays bilden je nach Präparat eine mechanische Barriere über der Blutung, erhöhen durch Absorption des Serums die Konzentration an Gerinnungsfaktoren, ziehen durch ihre negative Ladung positiv geladene Eiweiße aus der Blutbahn an und verstärken so die intrinsische Gerinnungskaskade.

TC 325 (Hemospray ${ }^{\mathrm{TM}}$ ), ein anorganisches, hoch absorptionsfähiges Pulver, bildet nach Kontakt mit Blut eine mechanische Barriere zur Versiegelung der Blutungsstelle. Durch Konzentration von Gerinnungsfaktoren soll zudem die Koagulationszeit verkürzt werden [213]. Es wird unter $\mathrm{CO}_{2}$-Insufflation aus $1-2 \mathrm{~cm}$ Entfernung direkt auf die Blutungsquelle aufgebracht. Erfahrungen liegen insbesondere in der Therapie von peptischen Ulzera vor. Hier liegen die primäre Hämostase (Fla/Flb-Läsionen) bei 92,8\% und die mittlere Blutungsrezidivrate bei 13,3\% [214]. TC 325 kann sowohl als Monotherapie als auch in Kombination mit anderen Verfahren, z. B. Hämoclips, verwendet werden [215]. Eine Fallserie von 60 Patienten mit unterschiedlichen Blutungsquellen im oberen und unteren Gastrointestinaltrakt beschreibt eine Hämostaserate von $98,5 \%$ und eine Rate von 9,5\% früher Blutungsrezidive ohne signifikante, unerwünschte Wirkungen [216].

Absorbierbare modifizierte Monomere aus Pflanzenstärke (Endoclot ${ }^{\mathrm{T} M}$ ) entziehen dem Blut Wasser, bilden eine gelartige Haftmasse und beschleunigen die Gerinnungskaskade. Über einen Applikationskatheter wird dieses Hämostasepulver in einem permanenten Luftstrom, der durch einen speziellen Kompressor erzeugt wird, ebenfalls aus $1-2 \mathrm{~cm}$ Abstand auf die Blutungsstelle aufgebracht. In einer Fallserie konnte bei allen 21 Patienten eine initiale Hämostase erreicht werden, mit einer Rezidivblutungsrate von 4,8\% innerhalb von 30 Tagen [217]. In einer weiteren Fallserie von 70 Patienten wurde im oberen Gastrointestinaltrakt in $64 \%$ der Fälle eine Blutstillung erreicht, wenn Endoclot ${ }^{\mathrm{TM}}$ primär zum Einsatz kam, und bei allen Patienten nach frustraner Hämostase mit anderen Verfahren. Im unteren Gastrointestinaltrakt lag die Hämostaserate bei $83 \%$. Blutungsrezidive ereigneten sich bei $11 \%(8 / 70)$ der Patienten [218].

Die Therapie mit anderen neuartigen Agentien, die z. B. bovine Gerinnungsfaktoren enthalten (SeraSeal ${ }^{\mathrm{TM}}$ ) oder dem synthetischen Aminosäuregel TDM-621, können derzeit außerhalb von Studien nicht empfohlen werden. Die Studienlage legt zwar nahe, dass deren Kombination mit Standardverfahren einen Mehrwert darstellen bzw. Rezidivblutungen nach Resektionen reduziert werden könnten, ausreichende Daten fehlen aber zum Zeitpunkt der Drucklegung [219, 220].

\section{Endoskopische Blutstillung in Abhängigkeit von der Blutungsursache}

\section{Ulkusblutung}

\section{EMPFEHLUNG 56}

Bei Hochrisikostigmata (Stadien: Fla, Flb, Flla) soll nach initialer Injektionstherapie ein zweites endoskopisches Hämostaseverfahren (mechanisch oder thermisch) erfolgen, um eine Rezidivblutung zu vermeiden.

Starke Empfehlung, starker Konsens.

Die Injektionstherapie dient der akuten Blutstillung und somit ggf. auch der Herstellung einer besseren Beurteilbarkeit der Blutungsquelle. Metaanalysen und eine Cochrane-Analyse zeigen, dass die Kombination der Injektion mit einem zweiten Hämostaseverfahren der alleinigen Injektionstherapie sowohl hinsichtlich der Rezidivblutungsraten als auch der Notwendigkeit einer chirurgischen Intervention signifikant überlegen ist und einen positiven Einfluss auf die Mortalität haben kann [199, 209-212].

In Fällen mit bereits initial sicherer Lokalisation der Blutungsquelle, insbesondere von Gefäßstümpfen, kann die mechanische oder thermische Blutstillung auch als Monotherapie erfolgen. Hämoclips und thermische Verfahren sind in den diesbezüglichen Studien überwiegend als gleichermaßen effektiv bewertet worden [199, 210 - 212].

\section{Mallory-Weiss-Läsionen}

\section{EMPFEHLUNG 57}

Zur endoskopischen Therapie relevanter Blutungen aus Mallory-Weiss-Läsionen des Ösophagus können Injektionstherapie und/oder mechanische Verfahren zum Wundrandverschluss verwendet werden.

Offene Empfehlung, starker Konsens. 
Sowohl durch Injektion von Epinephrin als auch durch Clipapplikation kann die Blutung aus Mallory-Weiss-Läsionen gestillt werden. Die Verwendung von Clips erlaubt den Wundrandverschluss [221 - 223]. Aufgrund der oft erhöhten Gewebefragilität in dieser Patientengruppe kann aber eine Clipapplikation durch mangelnden Halt der Clips erschwert sein oder die Situation aggravieren. Auch die Gummibandligatur kann zur Blutstillung in einer Mallory-Weiss-Läsion mit vergleichbarer Effektivität zum HämoclipVerschluss bzw. zur Injektionsbehandlung angewendet werden [224].

\section{Vaskuläre Malformationen}

\section{EMPFEHLUNG 58}

Angiektasien mit oder nach Blutung sollten primär durch ein thermisches Verfahren koaguliert werden.

Empfehlung, Konsens.

Die endoskopische Therapie einer Blutung bei GAVE-Syndrom kann durch ein thermisches Verfahren oder eine Gummibandligatur erfolgen. Bei Dieulafoy-Läsionen können neben thermischen Verfahren auch mechanische Verfahren angewandt werden.

Offene Empfehlung, Konsens.

Die Empfehlungen zur Endotherapie, z. B. Argon-Plasma-Koagulation, von Blutungen aus vaskulären Malformationen beruhen auf Fallserien, während kontrollierte, randomisierte Studien fehlen. So fand eine Übersichtsarbeit von 2014 anhand von 63 Studien nur geringe Evidenz für die endoskopische Therapie von Angiodysplasien [225]. Dennoch stellt die APC in der Regel eine etablierte Methode zur Beherrschung der akuten Blutung dar.

Gleichermaßen ist die APC weitverbreitetes Verfahren zur Therapie des GAVE-Syndroms. Allerdings fand sich in der erwähnten Übersichtsarbeit nach Ligaturtherapie eine signifikant höhere Rate an Patienten ohne Reblutungsereignis im Vergleich zur APC [225]. Vor diesem Hintergrund ist die Auswahl des Verfahrens aktuell der Expertise des Untersuchers überlassen, insbesondere bei ausgeprägten Befunden ggf. aber die Bandligatur vorzuziehen.

Ob mit der Radiofrequenzablation des GAVE-Syndroms ein weiteres Verfahren zur Auswahl steht, kann aktuell nicht beurteilt werden. Kleine Fallserien legen zumindest nahe, dass bei einem Teil der Patienten Hb-Stabilität bzw. ein verringerter Bedarf an Transfusionen erreicht werden kann [226-228].

Zur Therapie einer Ulkus-Dieulafoy-Blutung können Injektionsverfahren, thermische bzw. mechanische Methoden verwendet werden. Allerdings ist auch hier eine Kombination zu empfehlen, da die alleinige Injektionstherapie mit einem hohen Risiko für Rezidivblutungen vergesellschaftet ist [229]. Fulminante Blutungen profitieren ggf. vom Einsatz von OTSC zur Hämostase [230]. Auch die Wirksamkeit der endoskopischen Gummibandligatur wurde in einer kleinen Fallserie demonstriert [231].
Zur Therapie von Blutungen im Rahmen einer Strahlenproktitis ist insbesondere die APC etabliert [232], wenngleich vergleichende Studien vergleichbare Effektivität auch für die hyperbare Sauerstofftherapie [233] bzw. die bipolare Koagulation [234] zeigen.

\section{Blutung aus Malignomen}

\section{EMPFEHLUNG 59}

Eine Blutung aus Neoplasien im Gastrointestinaltrakt sollte primär endoskopisch therapiert werden.

Empfehlung, starker Konsens.

Zur endoskopischen Blutstillung von Malignomen im Gastrointestinaltrakt können Injektionsverfahren und/ oder thermische Methoden (z. B. Argon-Plasma- Koagulation) bzw., bei geeigneter Anatomie, auch beschichtete Stents zur mechanischen Kompression verwendet werden. Zu diesen Verfahren liegen Fallberichte bzw. Beobachtungen im Rahmen palliativer Tumorablation zur Stenosetherapie vor. Diese erfolgten insbesondere per ArgonPlasma-Koagulation oder bipolarer Koagulation [235 - 237]. Darüber hinaus kann die Blutstillung mittels Kompression durch einen beschichteten Metallstent gelingen [238]. Als Reserveverfahren kann auch hier ein Hämostasespray verwendet werden. So liegen in dieser Indikation z. B. Fallserien für TC-325 vor [216]. Bei Versagen der endoskopischen Blutstillung sollten weitere Verfahren (z. B. Embolisation, Radiatio, endoskopische/chirurgische Resektion) interdisziplinär abgestimmt und angewendet werden.

\section{Divertikelblutung}

\section{EMPFEHLUNG 60}

Die endoskopische Therapie einer akuten Divertikelblutung sollte mittels Injektion und/- oder mechanischer Verfahren erfolgen.

Empfehlung, starker Konsens.

Divertikelblutungen sistieren in der Regel spontan, sodass - nach Ausschluss anderer Blutungsquellen - die stattgehabte Blutung aus Kolondivertikeln als hochwahrscheinliche Verdachtsdiagnose gestellt wird. So zeigen Fallserien von Patienten mit Kolondivertikelblutung überwiegend spontane Hämostaseraten zwischen 77 und $92 \%$ [136, 239, 240]. Demzufolge ist die aktive, endoskopische Blutstillung relativ selten indiziert. Kann koloskopisch eine aktive Kolondivertikelblutung identifiziert werden, so ist eine endoskopische Hämostase durch Epinephrininjektion oder Hämoclipping bei über $75 \%$ der Patienten möglich [136, 241]. Auch für die Gummibandligatur und bipolare Koagulation liegen Fallberichte vor [136, 242]. 


\section{Blutungen während und nach endoskopischer Resektion}

\section{EMPFEHLUNG 61}

Zur endoskopischen Stillung intra- bzw. postprozeduraler Blutungen können Injektionsverfahren, thermische und mechanische Methoden zum Einsatz kommen.

Offene Empfehlung, starker Konsens.

Die intraprozedurale Hämostase erfolgt aufgrund fehlender vergleichender Studien allein basierend auf der Einschätzung des untersuchenden Arztes in Abhängigkeit von der individuellen Blutungsintensität, der Größe und Lage der Resektionsfläche, sowie dem Fortgang der Resektion. So kann im Verlauf größerer Resektionen eine thermische Koagulation blutender Gefäße, ggf. nach vorheriger Injektionstherapie, weniger hinderlich für den Fortgang der Abtragung sein als mechanische Verfahren [243]. Spezielle Koagulationsmodi wie z. B. die „soft coagulation“ unter Verwendung geeigneter Zangen oder eines passenden ESD-Messers gestatten eine in ihrer Intensität kontrollierbare Hämostase. So konnte im Rahmen der EMR großer Kolonadenome ( $n=198$, mittlere Größe 41,5 mm) für die „soft coagulation“ blutender Gefäße eine effektive Hämostase bei 91 \% der Fälle erzielt werden [244].

Auch Hämostasesprays werden zur Blutstillung im Rahmen von Resektionen verwendet. So liegen kleine Fallserien $(n=16)$ für TC-325 zur Hämostase während Resektionen vor [216]. Ein synthetisches Aminosäuregel (TDM-621) wurde bei 12 Patienten zur Blutstillung während Endoresektionen im Magen verwendet. Bei keinem der Patienten kam es zu Nachblutungen während des stationären Aufenthaltes [220].

Auch zur Therapie von postprozeduralen Blutungen fehlen vergleichende Studien. Analog zur Ulkusblutung ist davon auszugehen, dass mechanische und thermische Verfahren, ggf. in Kombination mit einer Injektionstherapie situationsabhängig angewendet werden sollten [245]. Die nach frischen großflächigen Resektionen ggf. diffuse Blutungssituation kann Anlass zur Anwendung von Hämostasesprays sein. Diesbezüglich liegen erste Fallberichte vor [220, 246, 247].

\section{Was sollte bei primärem Versagen der Blutstillung eingesetzt werden?}

\section{EMPFEHLUNG 62}

Bei primärem Versagen der Blutstillung können zusätzlich zu den konventionellen Verfahren je nach lokaler Expertise und Verfügbarkeit „over-the-scope clips“ (großvolumiger Clip zur Geweberaffung), hämostatische Pulver oder Injektionstechniken ggf. in Kombination eingesetzt werden.

Offene Empfehlung, starker Konsens (eine Enthaltung).
Publizierte Daten zu endoskopischen Reserveverfahren beim primären Versagen der Blutstillung beschränken sich derzeit auf Fallserien mit niedrigem Evidenzgrad. Ein „over-the-scope clip“ (OTSC) wurde in drei kleinen retrospektiven Studien bei Therapie-refraktären Blutungen im oberen und unteren Gastrointestinaltrakt mit einer Erfolgsrate zwischen 88 und $100 \%$ eingesetzt [206, 248]. Indikationen reichten von Ulzera und Dieulafoy- bzw. Mallory-Weiss-Läsionen über Divertikel- und Tumorblutungen bis zu post-interventionellen Blutungen nach endoskopischen oder chirurgischen Eingriffen. Als Nachteil der Methode muss die dafür notwendige Expertise angesehen werden, die derzeit nicht flächendeckend vorhanden ist. Eine prospektive randomisierte Studie an sieben deutschen Zentren, die allerdings erst in Abstract-Form vorliegt, zeigt bei der Rezidivblutung einen signifikanten Vorteil des OTSC gegenüber der Kombination zweier konventioneller Verfahren (Injektionstherapie und konventioneller Clip) [249].

Auch hämostatische Sprays wurden bereits bei refraktären Blutungen verschiedenster Art und Lokalisation im oberen und unteren Gastrointestinaltrakt mit einer Erfolgsrate zwischen 76 und $93 \%$ eingesetzt [250, 251]. Während die Applikation einfach und sicher erscheint, gibt es auch bei dieser Methode Limitationen aufgrund der Kosten und der Verfügbarkeit. Schließlich existieren eine Reihe von Injektionstechniken mit Koagulanzien oder Gewebeklebern, die bei refraktären Blutungen untersucht wurden.

Eine direkte Injektion der Blutungsquelle mittels Fibrin oder Thrombin wurde 2004 zwar in einer Stellungnahme der Amerikanischen Gesellschaft für Endoskopie (ASGE) positiv bewertet [204], findet aber in neueren Leitlinien aufgrund der Kosten und fehlender neuerer Daten im Vergleich zu anderen Techniken keine Berücksichtigung mehr [1, 252]. Bei fehlenden Alternativen kann das Verfahren jedoch lokal im Einzelfall noch zur Anwendung kommen. Als eher experimentell einzustufen sind dagegen neuere Untersuchungen zur lokalen Injektion von bovinen Gerinnungsfaktoren (SeraSeal ${ }^{\mathrm{TM}}$ ) oder die endosonografisch gesteuerte Embolisation der zuführenden Gefäße [219, 253].

\section{Wann sollten Biopsien entnommen werden? (inkl. H.p.-Diagnostik)}

\section{EMPFEHLUNG 63}

Bei Blutungen aus malignomsuspekten Läsionen kann die bioptische Sicherung in Abhängigkeit vom Aktivitätsgrad der Blutung und der Gerinnungssituation des Patienten bereits während der initialen Endoskopie erfolgen.

Offene Empfehlung, Konsens.

Besteht der V.a. auf das Vorliegen eines Malignoms als Blutungsquelle, so ist dieses bioptisch zu sichern. In Übereinstimmung mit den jeweiligen Leitlinien zur Diagnostik und Therapie von Ösophagus-, Magen- und kolorektalen Karzinomen sollten hierfür eine ausreichende Zahl an Proben entnommen werden. 


\section{EMPFEHLUNG 64}

Bei gastroduodenaler Ulkusblutung sollte bei vertretbarem Risiko bereits in der Notfallendoskopie eine bioptische Helicobacter-pylori-Diagnostik erfolgen. Empfehlung, starker Konsens.

Peptische Ulzera sind in hohem Maß mit der Besiedlung durch $\mathrm{H}$. pylori assoziiert [254, 255]. So ist auch das Risiko einer erneuten Ulkusblutung nach erfolgreicher Eradikationstherapie reduziert [256]. Bei positivem Befund soll daher eine Eradikationstherapie nach Wiederaufnahme der oralen Ernährung eingeleitet werden. Bei fehlendem H.-pylori-Nachweis sollte die Testung im Verlauf wiederholt werden. So ist die Rate falsch negativer H.-pylori-Testergebnisse z. B. höher, wenn die Diagnostik während der akuten Blutung erfolgt [256]. Eine Metaanalyse anhand von 8496 Patienten mit blutenden peptischen Ulzera fand eine H.-pylori-Prävalenz von $72 \%$, wobei die Infektionsrate signifikant höher war, wenn die Testung mindestens 4 Wochen nach dem Blutungsereignis erfolgte (OR 2,08, $95 \%$ KI 1,10-3,93; p = 0,024) [257]. Der Eradikationserfolg soll im Verlauf überprüft werden.

\section{Wann sollte eine offen-chirurgische oder radiologi- sche Intervention erwogen werden?}

\section{EMPFEHLUNG 65}

Eine offen-chirurgische oder radiologische Intervention kann erfolgen, bei a) technischem Versagen der endoskopischen Blutstillung inklusive Reserveverfahren; b) Rezidivblutung nach zweiter endoskopischer Intervention; c) endoskopisch nicht lokalisierbarer Blutungsquelle.

Offene Empfehlung, starker Konsens.

\section{EMPFEHLUNG 66}

Nach primär erfolgreicher endoskopischer Therapie kann bei hohem Rezidivrisiko eine angiografische oder operative Intervention erwogen werden.

Offene Empfehlung, starker Konsens.

\section{EMPFEHLUNG 67}

Die Auswahl des offen-chirurgischen oder radiologischen Verfahrens soll sich nach Art, Lokalisation und Schwere der Blutung, den Komorbiditäten des Patienten sowie der lokalen Expertise richten.

Starke Empfehlung, starker Konsens.

Eindeutige Indikationen für ein therapeutisches Alternativverfahren sind das technische Versagen der endoskopischen Blutstillung oder die endoskopisch nicht lokalisierbare Blutungsquelle. Mehrere randomisierte Studien und Metaanalysen zeigten dabei ebenso vergleichbare primäre Erfolgs- wie Mortalitätsraten der potenziellen radiologischen und chirurgischen Alternativen [258, 259].

Moderne radiologische Verfahren beruhen auf der super-selektiven angiografischen Embolisation mit primären Blutstillungsraten bis zu $90 \%$, interessanterweise auch bei lediglich endoskopisch und nicht angiografisch identifizierbarer Blutungsquelle [260 - 262]. Bei den chirurgischen Verfahren überwiegen heutzutage aufgrund der Notfallsituation mit Hochrisikopatienten die minimal-invasiven Eingriffe mit einer potenziell niedrigeren perioperativen Mortalität bei höheren Reblutungsraten [263, 264]. Im direkten Vergleich zeigen chirurgische Verfahren insgesamt eine höhere sekundäre Erfolgsrate mit weniger Reblutungen, während radiologische Verfahren auch bei schwerkranken Patienten mit sehr hohem Operationsrisiko eingesetzt werden können $[265,266]$.

Im Gegensatz zur Studienlage nach endoskopischem Therapieversagen beruht die Evidenz zur frühelektiven Intervention zur Prävention eines sekundären Therapieversagens auf historischen Daten mit nur einer randomisierten Studie [267]. Als Risikofaktoren für ein potenzielles endoskopisches Versagen ließen sich in den historischen Studien Größe $(>2 \mathrm{~cm}$ ) und Lage (duodenale Hinterwand) eines Ulkus, Alter (> 60 Jahre) und Komorbiditäten der Patienten sowie die kardiovaskuläre Stabilität während der Blutung herausfiltern [252, 264]. In diesen Fällen kann frühzeitig interdisziplinär eine Risikoabwägung und Therapieentscheidung erfolgen.

\section{Arbeitsgruppe 5: Therapie gastrointesti- naler Blutung bei portaler Hypertension}

\section{Wie ist die Definition der portalen Hypertension und einer Varizenblutung?}

Die portale Hypertension ist definiert als Erhöhung des Blutdrucks im portalen Stromgebiet, gemessen als hepatisch-venöser Druckgradient (HVPG), über $5 \mathrm{mmHg}$. Klinisch von Bedeutung ist jedoch das Vorliegen einer sogenannten klinisch-signifikanten portalen Hypertension (CSPH), definiert als portale Druckerhöhung über $10 \mathrm{mmHg}$. Diese Druckerhöhung ist von besonderer klinischer Relevanz, da sie die Grenze zwischen einem deutlich erhöhten Risiko u. a. auch für das Entstehen von Varizen markiert [268].

Eine Varizenblutung wird definiert als Blutung aus Ösophagusoder Magenvarizen zum Zeitpunkt der Endoskopie oder als Vorhandensein von größeren Varizen mit Blut im Magen ohne andere Blutungsquelle. Eine Blutung wird als klinisch signifikant gewertet, wenn der Bedarf an Blutkonserven mehr als 2 in 24 Stunden übersteigt, der initiale systolische Blutdruck $<100 \mathrm{mmHg}$ und die initiale Herzfrequenz über 100/min liegen [268]. Das Zeitfenster einer akuten Blutungsepisode beträgt 120 Stunden. Ein Versagen der Therapie zur Kontrolle einer aktiven Blutung ist definiert durch fortgesetztes Erbrechen von frischem Blut, Entwicklung eines hypovolämischen Schocks oder einen Abfall des Hämoglobins 
um $3 \mathrm{~g} / \mathrm{dl}(1,86 \mathrm{mmol} / \mathrm{I})$ innerhalb 24 Stunden ohne Gabe von Erythrozytenkonzentraten [26].

\section{Welche Blutungsquellen gibt es, in welcher Häufigkeit?}

Als potenzielle Blutungsquellen sollten bei Patienten mit portaler Hypertension zu den nicht-varikösen Blutungsquellen zusätzlich noch Varizen und die portal-hypertensive Gastropathie in Betracht gezogen werden. Varizen können im Ösophagus, im Magen, im gesamten Intestinum, im Kolon, im Rektum und peristomal an einem Anus praeter auftreten. Die Häufigkeit von Ösophagusvarizen liegt nach neueren Daten bei etwa $35 \%$ bei kompensierten Patienten und etwa $48 \%$ bei dekompensierten Patienten [269]. Gastrale Varizen traten in der Gesamtgruppe der zirrhotischen Patienten mit einer Häufigkeit von etwa 2 \% der Fälle auf [269]. Die kumulative Inzidenz bei Patienten ohne Varizen zum Zeitpunkt der Diagnose einer Leberzirrhose wurde in dieser Untersuchung mit $44 \%$ für 10 Jahre und 53 \% für 20 Jahre angegeben. Progression der Varizengröße wurde bei $33 \%$ der Patienten bei einer mittleren Beobachtungszeit von $145 \pm 109$ Monaten beobachtet [269].

Prävalenzzahlen für ektope Varizen, definitionsgemäß Varizen außerhalb von Ösophagus oder Magen, sind lediglich für kolorektale Varizen vorhanden. Die Prävalenz von kolorektalen Varizen wurde in der Literatur mit 32 bis $46 \%$ angegeben [270 - 272].

Andere Varizenlokalisationen sind sehr selten, wobei angenommen wird, dass in der überwiegenden Mehrzahl der Fälle die Kombination mit Ösophagus- bzw. Magenvarizen vorliegt [273]. Eine portal hypertensive Gastropathie ist nach den Daten der größten verfügbaren Studie (1016 Patienten) bei 37 \% der Patienten mit höhergradiger hepatischer Fibrose oder Zirrhose ohne vorherige gastrointestinale Blutung nachweisbar [274].

\section{Welche Besonderheiten sind bei Patienten mit Leber- zirrhose ohne Varizen und mit Varizen zur Verhinde- rung einer Varizenblutung (Primärprophylaxe) zu beachten?}

\section{EMPFEHLUNG 68A}

Jeder Patient mit Erstdiagnose einer Leberzirrhose soll zum Varizenscreening eine Gastroskopie erhalten.

Starke Empfehlung, starker Konsens.

\section{EMPFEHLUNG 68B}

Bei einer Lebersteifigkeit (transiente Elastografie) unter 20 kPa und einer Thrombozytenzahl über $150000 / \mu l$ kann auf die ÖGD verzichtet werden.

Offene Empfehlung, starker Konsens.

\section{EMPFEHLUNG 68C}

Die Reevaluation ist abhängig von dem Vorhandensein von Varizen und der Aktivität der Grunderkrankung und soll in einem Abstand von 1 bis 3 Jahren erfolgen.

Starke Empfehlung, starker Konsens.

\section{EMPFEHLUNG 68 D}

Zum Zeitpunkt der Dekompensation soll eine Gastroskopie durchgeführt werden.

Starke Empfehlung, Konsens.

Es wird empfohlen, bei jedem Patienten bei Erstdiagnose der Leberzirrhose eine Gastroskopie zur Evaluation auf Ösophagusvarizen durchzuführen. Eine Ausnahme kann bei Patienten mit einer Lebersteifigkeit (transiente Elastografie) unter $20 \mathrm{kPa}$ und einer Thrombozytenzahl über 150 000/ $\mu$ l gemacht werden, da diese Patienten ein sehr geringes Risiko für das Vorhandensein von Varizen haben [275, 276].

Einschränkend gilt, dass diese Daten aus einer retrospektiven Analyse und einer bisher nur in Abstraktform veröffentlichten Arbeit stammen. Eine Reevaluation mittels transienter Elastografie und Thrombozytenzahl sollte im jährlichen Abstand erfolgen, und bei Änderung der Parameter jenseits der oben genannten Grenzen sollte eine Gastroskopie erfolgen [268]. Die endoskopische Reevaluation ist abhängig von der Aktivität der Grunderkrankung [268]. Bei Dekompensation der Leberzirrhose wird eine erneute Gastroskopie zum Varizenscreening empfohlen [277]. Sind in der Indexgastroskopie keine Varizen vorhanden, ist eine Regastroskopie abhängig vom Stadium der Grunderkrankung nach 1 bis 3 Jahren empfohlen ( $\triangleright$ Tab. 7). Sind Varizen vorhanden, hängt das Untersuchungsintervall vom Stadium der Leberzirrhose (kompensiert vs. dekompensiert) und von der Größe der Varizen ab. Kompensierte Patienten mit therapierter Grunderkrankung oder fehlender Aktivität und fehlenden Kofaktoren sowie kleinen Varizen $(<5 \mathrm{~mm})$ sollten alle 2 Jahre untersucht werden. Bei Patienten mit kompensierter Erkrankung und nicht-therapierter Grunderkrankung sowie bei dekompensierten Patienten wird eine Kontrollgastroskopie jährlich empfohlen. Alle Patienten mit großen Varizen sollten einer Primärprophylaxe zugeführt werden und benötigen keine weitere endoskopische Screeninguntersuchung.

Die Empfehlungen sind in $>$ Tab. 7 zusammengefasst.

Welche Therapieformen zur Verhinderung einer Varizenblutung (Primärprophylaxe) gibt es? Wann sollten die unterschiedlichen Therapien durchgeführt werden?

\section{EMPFEHLUNG 69}

Bei Patienten mit Leberzirrhose ohne Nachweis von Varizen soll keine spezifische Therapie zur Verhinderung einer Entstehung von Varizen erfolgen.

Starke Empfehlung, starker Konsens. 
- Tab. 7 Empfehlungen zum Varizenscreening für Patienten mit Zirrhose.

\begin{tabular}{|l|l|l|}
\hline Gruppe & Varizen & empfohlener Zeitraum \\
\hline $\begin{array}{l}\text { kompensierte Zirrhose mit therapierter } \\
\begin{array}{l}\text { Grunderkrankung oder fehlender Aktivität } \\
\text { und fehlenden Kofaktoren (z. B. Übergewicht) }\end{array}\end{array}$ & Keine & 3 Jahre \\
\hline $\begin{array}{l}\text { kompensierte Zirrhose mit nicht-therapierter } \\
\text { aktiver Grunderkrankung }\end{array}$ & keine & 2 Jahre \\
\hline $\begin{array}{l}\text { kompensierte Zirrhose mit therapierter } \\
\text { Grunderkrankung oder fehlender Aktivität } \\
\text { und fehlenden Kofaktoren (z. B. Übergewicht) }\end{array}$ & kleine Varizen $(<5 \mathrm{~mm})$ & 2 Jahre \\
\hline $\begin{array}{l}\text { kompensierte Zirrhose mit nicht-therapierter } \\
\text { aktiver Grunderkrankung }\end{array}$ & kleine Varizen $(<5 \mathrm{~mm})$ & jährlich \\
\hline \begin{tabular}{l} 
dekompensierte Patienten \\
\hline
\end{tabular} & mit oder ohne Varizen & jährlich \\
\hline
\end{tabular}

\section{EMPFEHLUNG 70}

Bei Patienten mit kleinen Varizen $(<5 \mathrm{~mm})$ und Nachweis von endoskopischen Blutungsbereitschaftszeichen (red spots oder red wale signs) oder im Child-Pugh-Stadium C sowie bei Patienten mit großen Varizen ( $>5 \mathrm{~mm}$ ) soll eine Primärprophylaxe zur Verhinderung einer Varizenblutung erfolgen.

Starke Empfehlung, starker Konsens.

\section{AUSSAGE 10}

Die endoskopische und die medikamentöse Therapieform sind in der Verhinderung einer Ösophagusvarizenblutung (Primärprophylaxe) gleichwertig.

Starker Konsens.

\section{EMPFEHLUNG 71}

Die medikamentöse Therapie soll durch einen nicht-selektiven Betablocker erfolgen.

Starke Empfehlung, starker Konsens.

\section{EMPFEHLUNG 72}

Zur medikamentösen Blutungsprophylaxe sollen Carvedilol oder Propranolol eingesetzt werden.

Starke Empfehlung, Konsens.

\section{EMPFEHLUNG 73}

Zur endoskopischen Therapie soll die Ligatur der Ösophagusvarizen erfolgen.

Starke Empfehlung, starker Konsens.
Bei Patienten mit Leberzirrhose ohne Varizen wird eine medikamentöse Therapie nicht empfohlen. Diese Empfehlung beruht auf den Daten einer großen internationalen Plazebo-kontrollierten Multicenterstudie [278, 279]. In dieser Plazebo-kontrollierten Multicenterstudie wurde bei Patienten mit Leberzirrhose ohne Varizen die Wirksamkeit einer prophylaktischen medikamentösen Therapie mit einem nicht-selektiven Betablocker untersucht. Die Ergebnisse zeigen sowohl in Bezug auf das Auftreten einer Varizenblutung als auch auf das Überleben keine Unterschiede zwischen der Gruppe mit Betablocker-Therapie verglichen mit der Plazebo-Gruppe [278]. Zudem zeigt die Metaanalyse, dass auch Patienten mit kleinen Varizen nicht von einer Betablocker-Therapie profitieren. Die Therapie mit einem Betablocker verhinderte nicht das Entstehen von großen Varizen $(O R=1,05,95 \% \mathrm{KI}$ : $0,25-4,36 ; p=0,95)$ oder eine erste Varizenblutung $(O R=0,59$, $95 \% \mathrm{Kl}: 0,24-1,47 ; \mathrm{p}=0,26)$ und verbesserte das Überleben nicht $(O R=0,70,95 \%$ KI: 0,45-1,10; $p=0,12)$ [279]. Beide Studien weisen allerdings eine Zunahme von Nebenwirkungen $(\mathrm{OR}=3,47,95 \% \mathrm{KI}: 1,45-8,33 ; \mathrm{p}=0,005)$ in der BetablockerGruppe aus. Eine aktuelle randomisierte Studie zeigt demgegenüber eine niedrigere Progressionsrate von kleinen zu großen Varizen bei einer Therapie mit Carvedilol [280]. Eine nachfolgende Metaanalyse unter Berücksichtigung dieser Daten zeigt allerdings keinen Vorteil für eine Betablocker-Therapie, sodass aktuell keine eindeutige Empfehlung zur Therapie gegeben werden kann [281]. Bei Patienten mit kleinen Varizen $(<5 \mathrm{~mm})$ und endoskopischen Blutungsbereitschaftszeichen wie red spots oder red wale signs oder im Child-Pugh-Stadium C sowie Patienten mit großen Varizen (> $5 \mathrm{~mm}$ ) wird eine Primärprophylaxe zur Verhinderung einer Varizenblutung empfohlen [268, 282]. Zur Verfügung stehen medikamentöse und endoskopische Therapieformen mit vergleichbaren Erfolgsergebnissen [268, 283]. Die empfohlene medikamentöse Behandlungsform ist eine Therapie mit einem nichtselektiven Betablocker, normalerweise Propranolol [284] oder Carvedilol [285]. Eine Metaanalyse mit 1859 Patienten aus 20 Studien verglich 931 Patienten mit Propranololtherapie mit 928 Patienten als Kontrollgruppe. Das gepoolte Risiko für eine Blutung $(-11 \%$ [95\% KI, $-21 \%,-1 \%])$ und die Mortalität $(-9 \%$ [95\% KI, $-18 \%,-1 \%])$ bei Patienten unter Propranololtherapie in der 
Primärprophylaxe waren signifikant geringer verglichen mit der Kontrollgruppe [286]. Es wurde in randomisierten Vergleichsstudien und in Metaanalysen gezeigt, dass Carvedilol in diesem Zusammenhang ein größeres portaldrucksenkendes Potenzial verglichen mit den klassischen nicht-selektiven Betablockern (Propranolol, Nadolol) hat [287, 288]. Interessant ist eine neuere Studie, die den Effekt von Carvedilol nach hämodynamisch definiertem Versagen (Kriterien: Senkung des HVPG unter $12 \mathrm{mmHg}$ oder um $20 \%$ ) einer Propranolol-Therapie untersucht hat [289]. Danach zeigt ein erheblicher Teil der Patienten, die nicht auf eine Propranololtherapie angesprochen haben, ein Ansprechen auf eine Carvediloltherapie, was wiederum mit einer Senkung der Reblutungsrate, einer geringeren hepatischen Dekompensation und schließlich zu höheren Überlebensraten führte.

Die Empfehlungen zur Primärprophylaxe sollten auch bei Patienten mit Aszites oder therapierefraktärem Aszites angewandt werden. Die Ergebnisse einer retrospektiven Studie mit schlechterem Überleben bei Patienten mit therapierefraktärem Aszites [290] konnten in neueren Untersuchungen nicht bestätigt werden. Eine retrospektive Untersuchung an einer großen Patientenzahl konnte sogar einen Überlebensvorteil für Patienten mit Aszites und therapierefraktärem Aszites und Betablocker-Therapie zeigen [291]. Eine prospektive Untersuchung konnte einen Überlebensvorteil von Patienten mit Betablocker-Therapie und akutauf-chronischem Leberversagen aufzeigen [292]. Damit können die Empfehlungen zur Primärprophylaxe auch für Patienten mit Aszites und therapierefraktärem Aszites Anwendung finden.

Ziel in der Primärprophylaxe ist es, den portalen Druck auf unter $12 \mathrm{mmHg}$ zu senken oder eine Reduktion um $10 \%$ zu erreichen [268, 293]. Aufgrund der invasiven Methode zur Messung des portalen Drucks (Lebervenenverschlußdruckmessung) ist die Kontrolle des Therapieerfolgs allerdings in der Praxis schwierig zu evaluieren. Es wird daher zur Einstellung die maximal tolerierbare Dosis, eine Senkung der Herzfrequenz auf 50 - 55/min bzw. die maximale Dosis (Propranolol $320 \mathrm{mg} / \mathrm{d}$; Carvedilol 12,5 $25 \mathrm{mg} / \mathrm{d}$ ) empfohlen [289].

Als Alternativtherapie, bei Versagen der medikamentösen Therapie oder Unverträglichkeit bzw. Kontraindikationen für die Einnahme eines Betablockers wird die endoskopische Behandlung von blutungsgefährdeten Varizen empfohlen [268, 282]. Die empfohlene Methode ist in diesem Zusammenhang die Varizenligatur. Die Empfehlung beruht auf mehreren Metaanalysen und randomisierten Studien, die die Betablocker-Therapie mit der Varizenligatur bei blutungsgefährdeten (high risk) Varizen miteinander verglichen haben. In diesen Untersuchungen zeigen sich beide Behandlungsformen gleichwertig in der Verhinderung einer Ösophagusvarizenblutung [283, 294 - 296].

\section{EMPFEHLUNG 74}

Eine Kombination von medikamentöser und endoskopischer Therapie soll zur Primärprophylaxe nicht durchgeführt werden.

Starke Empfehlung, Konsens.
Die Kombination aus Betablocker-Therapie und Varizenligatur wird in der Primärprophylaxe zur Verhinderung einer Varizenblutung nicht empfohlen. Diese Empfehlung beruht auf einer multizentrischen randomisierten Studie, in der Patienten im Rahmen der Primärprophylaxe einerseits mit Betablocker allein und andererseits mit Betablocker und endoskopischer Varizenligatur verglichen wurden [297]. Die Ergebnisse zeigten eine Zunahme von Komplikationen in der Ligatur-Gruppe, sodass eine Kombinationstherapie in der Primärprophylaxe nicht empfohlen werden kann.

Es gibt nur sehr wenige bzw. keine Daten in der Primärprophylaxe bei Fundus- oder ektopen Varizen, sodass eine Primärprophylaxe bei diesen Varizen analog der Empfehlungen der Ösophagusvarizen durchgeführt werden sollte.

\section{EMPFEHLUNG 75A}

Patienten mit einem Budd-Chiari-Syndrom oder einer Thrombose der Pfortader bzw. Mesenterialgefäßen sollen neben einer Antikoagulation eine Therapie zur Verhinderung einer Varizenblutung erhalten.

Starke Empfehlung, starker Konsens.

\section{EMPFEHLUNG 75B}

Als Therapien sollten die medikamentöse Therapie mit einem nicht-selektiven Betablocker, die endoskopische Therapie mit Ösophagusvarizenligatur oder die Implantation eines transjugulären portosystemischen Stents (TIPS) zum Einsatz kommen.

Empfehlung, starker Konsens (eine Enthaltung).

Erstmaßnahme bei Patienten mit Budd-Chiari-Syndrom, Pfortader- oder splanchnischer Thrombose ist die Antikoagulation. Eine Prophylaxe zur Verhinderung einer Varizenblutung ist bei allen Patienten parallel zur Antikoagulation empfohlen [268]. Aufgrund fehlender Daten ist allerdings unklar, ob die medikamentöse oder die endoskopische Therapie in der Primärprophylaxe vorteilhafter ist. Es wird daher empfohlen, die obengenannten Empfehlungen für die Ösophagusvarizen anzuwenden. Zusätzlich sollte bei allen Patienten die Möglichkeit der Anlage eines transjugulären portosystemischen Shunts (TIPS) geprüft werden. Die Datenlage zur Implantation eines TIPS bei Patienten mit BuddChiari-Syndrom ist allerdings gering. In einer retrospektiven Analyse mit 67 Patienten zeigte sich sowohl für beschichtete als auch für nicht beschichtete Stents ein exzellentes Kurzzeit- als auch Langzeitüberleben (12 Monate: 92\%; 120 Monate: 72\%) [298]. Erwartungsgemäß war die Offenheitsrate bei beschichteten Stents höher im Vergleich zu nicht beschichteten Stents (76 vs. $27 \%$ ), welches wiederum eine geringe Reinterventionsrate bei Patienten mit einem beschichteten Stent zur Folge hatte (22 vs. $100 \%)$ [298]. 


\section{EMPFEHLUNG 75C}

Eine portaldrucksenkende Therapie kann bei Patienten mit stark ausgeprägten portal-hypertensiver Gastropathie durchgeführt werden.

Offene Empfehlung, starker Konsens.

\section{EMPFEHLUNG 75 D}

Bei Patienten mit gering ausgeprägter portal-hypertensiver Gastropathie soll eine portaldrucksenkende Therapie nicht durchgeführt werden.

Starke Empfehlung, starker Konsens.

Bei Patienten mit einer portal-hypertensiven Gastropathie wird eine Primärprophylaxe abhängig vom Schweregrad der Ausprägung empfohlen. Sind die Magenveränderungen gering ausgeprägt, ist der Vorteil einer portaldrucksenkenden Therapie nicht belegt. Bei starker Ausprägung können Patienten von einer medikamentösen portaldrucksenkenden Therapie mit nicht-selektiven Betablockern profitieren. Diese Empfehlung basiert auf den Empfehlungen zur Primärprophylaxe der Ösophagusvarizen, da Daten bei portal-hypertensiver Gastropathie in der Primärprophylaxe nicht vorhanden sind. Sollte eine Kombination aus Ösophagusvarizen und portal-hypertensiver Gastropathie vorliegen, ist eine Primärprophylaxe analog der Empfehlungen zu den Ösophagusvarizen anzuraten.

\section{Wie ist der Schweregrad der Blutung (endoskopisch) einzuschätzen?}

\section{EMPFEHLUNG 76}

Der Schweregrad der gastrointestinalen Blutung im Rahmen einer portalen Hypertension sollte klinisch, laborchemisch und anhand der systemischen Hämodynamik eingeschätzt werden (siehe hierzu Empfehlungen der AG 1). Als endoskopische Risikofaktoren für das erneute Auftreten einer Varizenblutung sollten eine aktive Blutung zum Zeitpunkt der Endoskopie, das Vorhandensein von großen Ösophagus- und Magenvarizen, red color sign auf den Varizen und ein Koagel auf der Varize verwendet werden.

Empfehlung, starker Konsens.

Der Schweregrad wird bei Blutungen aufgrund eines portalen Hypertonus normalerweise nicht-endoskopisch eingeschätzt. Dabei werden sowohl der Hämoglobinwert und der Hämatokritwert als auch die systemische Hämodynamik als Kriterien der Schwere der Blutung herangezogen (siehe Kapitel Initiale Risikoeinschätzung der AG1). Die Verwendung von prädiktiven Scoresystemen wird zum jetzigen Zeitpunkt nicht empfohlen. Entsprechend konnte in einer aktuell veröffentlichten prospektiven Untersuchung keine statistisch signifikante Vorhersage in Bezug auf den klinischen Verlauf und die Mortalität bei Patienten mit einer Varizenblutung gefunden werden [299]. Ergebnisse einer randomisierten Multicenterstudie zeigten, dass vor allem bei Patienten mit Leberzirrhose im Stadium Child-Pugh A und B die Gefahr einer Übertransfusion besteht [300]. Mit einem restriktiven Transfusionsschema (Transfusion bei Abfall des Hämoglobinwertes unter $7 \mathrm{~g} / \mathrm{dl}$ ) zeigten diese Patienten ein signifikant besseres Überleben (HR 0,30; $95 \%$ KI 0,11-0,85) und eine niedrigere Reblutungsrate als bei liberalem Transfusionsschema (Transfusion bei Abfall des Hämoglobinwertes unter $9 \mathrm{~g} / \mathrm{dl}$ ), während im Stadium Child-Pugh C $(1,04 ; 95 \%$ KI 0,45 - 2,37) beide Strategien kein unterschiedliches Überleben zeigten. Entsprechend war die Notwendigkeit zur Anlage einer Sengstakensonde bzw. eines transjugulären intrahepatischen portosystemsichen Shunts seltener in der restriktiven Transfusionsgruppe [300].

Endoskopische Risikofaktoren für eine frühe Reblutung sind der Nachweis einer aktiven Blutung während der Endoskopie bzw. das Vorliegen von oder eine Blutung aus gastralen Varizen [301, 302]. Große Varizen, red color signs auf den Varizen oder ein Koagel auf Varizen sind ebenfalls mit einer höheren Reblutungsrate korreliert [301, 302]. Nicht-endoskopische Faktoren, die mit einer frühzeitigen Reblutung assoziiert sind, sind Alter über 60 Jahre, alkoholische Leberzirrhose, schwere Initialblutung mit einem Hb-Abfall auf unter $8 \mathrm{~g} / \mathrm{dl}$, Thrombozytopenie, Enzephalopathie, Aszites, hoher hepatisch-venöser Druckgradient und Nierenversagen [302-304].

\section{Welche therapeutischen Besonderheiten sind bei Blutungen im Rahmen einer portalen Hypertension zu beachten? Welche medikamentösen Therapieformen gibt es? Wann sollten die medikamentösen Therapien durchgeführt werden?}

\section{EMPFEHLUNG 77}

Bei Verdacht auf eine Varizenblutung soll die medikamentöse Therapie mit einem Vasopressin-Analogon und einem Antibiotikum begonnen werden. Es soll eine Kombination aus medikamentöser und endoskopischer Therapie erfolgen. Die endoskopische Untersuchung soll so zeitnah wie möglich durchgeführt werden, spätestens jedoch nach 12 Stunden. Starke Empfehlung, starker Konsens.

\section{EMPFEHLUNG 78}

Nach erfolgreicher primärer Blutstillung sollte bei Patienten mit Risikofaktoren (Child-Pugh-Score B mit aktiver Blutung, Child-Pugh-Score $C \leq 13$ ) die frühzeitige Anlage (innerhalb von $72 \mathrm{~h}$ ) eines transjugulären portosystemischen Shunts (TIPS) erwogen werden.

Empfehlung, Konsens (eine Enthaltung). 


\section{EMPFEHLUNG 79A}

Ein analoges Vorgehen mit Gabe von Vasopressin-Analogon und Antibiotikagabe sollte bei einer Blutung im Rahmen einer portal-hypertensiven Gastropathie durchgeführt werden. Empfehlung, starker Konsens.

\section{EMPFEHLUNG 79B}

Zusätzlich können diese Patienten eine Therapie mit einem Protonenpumpenhemmer erhalten.

Offene Empfehlung, starker Konsens.

Eine therapeutische Besonderheit bei Blutungen im Rahmen einer portalen Hypertension ist der Therapiestart verschiedener medikamentöser Regime bereits bei vermuteter Blutung (siehe Kapitel Medikamentöse Therapie der AG1). Die medikamentöse vasoaktive Therapie sollte bereits bei vermuteter Varizenblutung gestartet werden und während der endoskopischen Erstuntersuchung fortgeführt werden. Die Therapie kann mit Terlipressin (1 - 2 mg alle 4 Stunden i.v.), Somatostatin ( $250 \mathrm{mcg}$ Bolus, $250 \mathrm{mcg} / \mathrm{h}$ oder $500 \mathrm{mcg} / \mathrm{h}$ i. v. Infusion) oder Octreotid $(25-50 \mathrm{mcg} / \mathrm{h} \mathrm{i.v.}$ Infusion) durchgeführt werden (siehe Kapitel Medikamentöse Therapie in der AG1) [72]. Nach Einleitung der medikamentösen Therapie sollte immer eine endoskopische Therapie durchgeführt werden. Die Durchführung der Notfallendoskopie sollte so zeitnah wie möglich erfolgen, wobei nach internationalen Maßstäben dabei innerhalb der ersten 12 Stunden als Zeitgrenze angesehen wird [268]. Wichtig ist die Kombination aus einer medikamentösen und einer endoskopischen Therapie. Die Empfehlung beruht auf den Ergebnissen einer Metaanalyse, die einen signifikanten Vorteil für eine Kombinationstherapie verglichen mit einer alleinigen endoskopischen Therapie zeigte [28]. Die Kombinationstherapie ging einher mit einer besseren primären Blutstillung (HR 1,12; 95 \% KI: 1,02-1,23) und einer niedrigeren Rate an Reblutungen innerhalb der ersten 5 Tage nach Initialblutung (HR 1,28; $95 \%$ KI: 1,18-1,39). Die maximale medikamentöse Therapiedauer der Vasopressin-Analogon -Therapie beträgt bei allen aufgeführten Medikamenten fünf Tage. Analog der Weiterführung der Vasopressin-Analogon -Therapie wird auch die Weiterführung der antibiotischen Therapie für sieben Tage nach endoskopischer Versorgung empfohlen.

Patienten mit einem hohen Risiko einer Reblutung (ChildPugh-Score B mit aktiver Blutung, Child-Pugh-Score $C \leq 13$ ) profitieren von einer frühzeitigen Anlage (innerhalb von $72 \mathrm{~h}$ ) eines transjugulären portosystemischen Shunts (TIPS) [305, 306]

Ein analoges Vorgehen mit Gabe eines Antibiotikums und Vasopressin-Analogon wird im Rahmen einer aktiven Blutung bei portal-hypertensiver Gastropathie empfohlen. Weiterhin können diese Patienten eine Therapie mit einem Protonenpumpenhemmer erhalten. Diese Empfehlungen beruhen auf den Ergebnissen von zwei randomisierten Studien [307, 308]. In der ersten doppelblind randomisierten Multicenterstudie bei 86 Patienten mit Blutung aus einer portal-hypertensiven Gastropathie oder Varizen hatten die Patienten mit einer höheren Dosis und längeren Applikationszeit von Terlipressin ( $1 \mathrm{mg}$ alle 4 Stunden über 5 Tage) eine bessere Kontrolle der Blutung als Patienten mit einer kürzeren Applikationszeit und niedrigeren Dosierung des Terlipressins (0,2 mg alle 4 Stunden über 2 Tage) [307].

Die zweite randomisierte Studie hat die Effektivität einer Therapie mit Octreotid, Vasopressin und Omeprazol bei 68 Patienten verglichen. Die Blutung wurde kontrolliert bei $100 \%$ der Patienten in der Octreotid-Gruppe, bei $64 \%$ der Patienten in der Vasopressin-Gruppe und bei $59 \%$ der Patienten in der Omeprazol-Gruppe [308].

\section{Welche (endoskopischen) Blutstillungsverfahren gibt es?}

\section{AUSSAGE 11}

Als endoskopische Blutstillverfahren stehen die Varizenligatur und die Sklerotherapie mit Injektion von n-Butyl-2-Cyanoacrylat zur Verfügung. Bevorzugte Methode ist die Ligaturtherapie. Bei Versagen der endoskopischen Blutstillverfahren stehen die Anlage eines beschichteten Metallstents oder die Anlage von Sengstaken- bzw. Linton-Nachlas-Sonden zur Verfügung.

Starker Konsens.

\section{EMPFEHLUNG 80A}

Nach Einsatz dieser Bridgingverfahren sollte immer die Möglichkeit der Implantation eines TIPS geprüft werden.

Empfehlung, starker Konsens.

Als endoskopische Blutstillverfahren stehen die Varizenligatur und die Sklerotherapie mit Injektion von n-Butyl-2-Cyano-acrylat zur Verfügung. Bevorzugte Methode ist die Ligaturtherapie. Die Sklerotherapie sollte nur bei Versagen der Ligaturtherapie zum Einsatz kommen. Zu der technischen Durchführung dieser Methoden wird auf die DGVS-Leitlinie: S2k-Leitlinie Qualitätsanforderungen in der gastrointestinalen Endoskopie, AWMF-Register Nr. 021 022 verwiesen. Weitere endoskopische Verfahren zur Blutstillung sind die Einlage eines vollbeschichteten, selbst expandierenden Metallstents (z. B. Ella-Danis-Stent) oder die Einlage einer Sengstaken- (Ösophagusvarizenblutung) bzw. Linton-Nachlas-Sonde (gastrische Varizen). Diese Verfahren sollten nur bei Versagen der Ligatur und Sklerotherapie eingesetzt werden und werden unten (Empfehlung 81) erläutert.

\section{EMPFEHLUNG 80B}

Bei medikamentös nicht beherrschbarer Blutung oder rezidivierender Blutung bei portal-hypertensiver Gastropathie sollte die TIPS-Implantation geprüft werden. Empfehlung, starker Konsens (eine Enthaltung). 
Bei Patienten mit einer medikamentös nicht beherrschbaren Blutung oder bei Patienten mit Rezidivblutung bei portal-hypertensiver Gastropathie sollte die Möglichkeit einer TIPS-Implantation geprüft werden. Die Daten über die Effektivität einer TIPS-Anlage bei dieser Patientengruppe sind allerdings limitiert. In einer Studie wurden 40 Patienten mit gering und stark ausgeprägter portalhypertensiver Gastropathie einer TIPS-Implantation zugeführt [309]. Die TIPS-Implantation war assoziiert mit einer endoskopischen Verbesserung und einer Abnahme der Transfusionshäufigkeit bei 89 bzw. 75 \% der Patienten mit gering bzw. stark ausgeprägter portal-hypertensiver Gastropathie [309].

\section{Wie ist die Definition des primären Versagens? Was sollte bei primärem Versagen der Blutstillung einge- setzt werden?}

\section{AUSSAGE 12}

Das primäre Versagen der Blutstillung bei Varizenblutung ist definiert als Therapieversagen der medikamentösen und endoskopischen Therapie mit fortgesetzter Blutung (Erbrechen von frischem Blut), fehlender hämodynamischer Stabilisierung, erneuter Abfall des Hämoglobinwertes um $3 \mathrm{~g} / \mathrm{dl}$ oder Reblutung in den ersten fünf Tagen.

Starker Konsens.

\section{EMPFEHLUNG 81}

Bei refraktärer Ösophagusvarizenblutung sollte primär ein beschichteter, selbst expandierender Metallstent eingelegt werden. Alternativ oder bei Fundusvarizenblutung sollten Sonden (Sengstaken-Sonde, Linton-Nachlas-Sonde) als Überbrückungstherapie eingelegt werden.

Empfehlung, starker Konsens.

Das primäre Versagen ist definiert als Versagen der medikamentösen und endoskopischen Therapien mit fortgesetzter Blutung, fehlende hämodynamische Stabilisierung oder Reblutung in den ersten fünf Tagen. Ein Versagen der Therapie zur Kontrolle einer aktiven Blutung ist definiert durch fortgesetztes Erbrechen von frischem Blut, Entwicklung eines hypovolämischen Schocks oder Abfall des Hämoglobins um $3 \mathrm{~g} / \mathrm{dl}(1,86 \mathrm{mmol} / \mathrm{l})$ in 24 Stunden ohne Gabe von Erythrozytenkonzentraten [26]. In der Akutphase steht zunächst die erneute Blutstillung und hämodynamische Stabilisierung im Vordergrund. Bei Versagen der Ligatur und Sklerosierungstherapie stehen als weitere Therapieverfahren die Einlage eines vollbeschichteten, selbstexpandierenden Metallstents und die Einlage einer Sengstaken- (Ösophagusvarizenblutung) bzw. Linton-Nachlas-Sonde (Fundusvarizen-Blutung) zur Verfügung. Empfohlen wird als Erstmaßnahme die Einlage eines selbstexpandierten Metallstents. In einer aktuellen randomisierten Multicenterstudie konnte eine signifikant höhere Kontrolle der Blutung (85 vs. $47 \%$ ) durch den Metallstent (SX-Ella-Stent Danis) gezeigt werden [310]. Vergleichbare Ergebnisse mit höherer Kontrolle der Blutung bei Verwendung eines Stents zeigt eine im Druck befindliche Metaanalyse [311]. Für die technische Durchführung wird auf die DGVS-Leitlinie: S2k-Leitlinie Qualitätsanforderungen in der gastrointestinalen Endoskopie, AWMF-Register Nr. 021 022 verwiesen.

Beide Therapieverfahren, Stenteinlage oder Sondenanlage, sind zeitlich limitierte Verfahren (Stent: bis 14 Tage; Sonden: 24 Stunden). In einer neueren Studie wurden die Stents im Mittel nach 11 Tagen entfernt [312]. Die Studie zeigte weiterhin eine hohe Reblutungsrate nach Entfernen des Stents, sodass beide Therapieformen als vorübergehende Maßnahmen anzusehen sind. Eine neuere Studie hat die Möglichkeit der Blutstillung durch hämostatische Puder untersucht [313]. Die Ergebnisse zeigen die Möglichkeit der kurzfristigen Blutstillung, eine Empfehlung kann aber aufgrund der niedrigen Patientenzahl nicht gegeben werden. Grundsätzlich sollte bei allen Patienten die Möglichkeit zur TIPSAnlage geprüft werden.

\section{EMPFEHLUNG 82}

Bei primärem Therapieversagen und fehlenden Kontraindikationen sollte ein transjugulärer intrahepatischer portosystemischer Shunt (TIPS) implantiert werden.

Empfehlung, starker Konsens (eine Enthaltung).

Die Empfehlung zur frühen TIPS-Anlage basiert auf den Ergebnissen mehrerer Metaanalysen und randomisierter Studien. Eine hochrangig publizierte randomisierte, kontrollierte Studie [305] zeigte den Nutzen des frühen TIPS bei Hochrisikopatienten (Def. s. nächster Absatz) mit akuter Varizenblutung. Der Einsatz e-PTFE-beschichteter Stents führte zu hohen Offenheitsraten, signifikant erniedrigter Rezidivblutungsrate, niedriger Mortalität und resultierte in einer hohen Rate kontrollierbarer Blutungen. Das Risiko einer hepatischen Enzephalopathie war hingegen nicht erhöht. Eine weitere Studie derselben Arbeitsgruppe konnte die Ergebnisse bestätigen [306].

In Metaanalysen wurden zirrhotische Patienten mit akuter Varizenblutung (bei drei Studien Ösophagusvarizenblutung, bei einer Studie gastrische Varizenblutung und in den restlichen Studien eine Kombination aus Ösophagusvarizen- und/oder gastrische Varizenblutung) verglichen [314]. Zusätzlich wurden in drei Studien ausschließlich Hochrisikopatienten (mit einem erhöhten Lebervenenverschlußdruck [HVPG] über $20 \mathrm{mmHg}$, Patienten mit Child-Pugh 10 - 13 Punkten bzw. 7 - 9 Punkten und aktiver Blutung) eingeschlossen. Die Ergebnisse zeigen einen Vorteil in der Reblutungsrate, beim Gesamtüberleben und in der Verhinderung eines Blutungs-assoziierten Todes in der TIPS-Gruppe im Vergleich zum ausschließlich endoskopisch-medikamentösen Vorgehen. Die Entwicklung einer Enzephalopathie war nicht signifikant unterschiedlich zwischen beiden Interventionsgruppen. In Bezug auf die Gesamtblutungsrate zeigte sich bei Berücksichtigung aller eingeschlossenen Studien kein Unterschied zwischen beiden Interventionsgruppen. Hierzu ist allerdings anzumerken, dass die Heterogenität der Studien signifikant war. Nach Ausschluss einer Studie zeigte sich keine signifikante Heterogenität mehr und ein 
Vorteil für die Behandlung mit einem TIPS [314]. Zu denselben Ergebnissen mit Vorteil für die TIPS-Implantation und fehlender höherer Enzephalopathierate kommt eine weitere aktuelle Metaanalyse [315].

\section{Wie ist die Definition des sekundären Versagens? Was sollte bei sekundärem Versagen der Blutstillung eingesetzt werden?}

\section{AUSSAGE 13}

Als Sekundärversagen ist eine erneute Varizenblutung mindestens 5 Tage nach Blutstillung definiert.

Starker Konsens.

\section{EMPFEHLUNG 83}

Das Sekundärversagen soll in der Akutphase wie eine initiale Blutung therapiert werden.

Starke Empfehlung, starker Konsens.

\section{EMPFEHLUNG 84}

Nach Sekundärversagen sollte bei fehlenden Kontraindikationen ein transjugulärer intrahepatischer portosystemischer Shunt (TIPS) implantiert werden.

Starke Empfehlung, starker Konsens (eine Enthaltung).

Als Sekundärversagen ist eine Blutung nach stattgehabter Varizenblutung mit einem Abstand von mindestens 5 Tagen nach vorheriger Blutung definiert.

Für die Sekundärprophylaxe weisen TIPS und die endoskopische Therapie vergleichbare Überlebensraten auf. Dies wurde durch zahlreiche randomisierte Studien [316-321] belegt sowie in Expertenkommissionen konsentiert. Die Rate der hepatischen Enzephalopathien war aber in den meisten Studien in der TIPSGruppe höher und sollte in die therapeutischen Überlegungen einbezogen werden. Die neuesten Ergebnisse kommen aus einer deutschen randomisierten Multicenterstudie [322]. Dabei wurden die Patienten frühestens fünf Tage nach vorheriger Varizenblutung in einen Interventionsarm mit TIPS-Implantation (e-PTFE-beschichteter Stent, 8 mm Durchmesser) oder einen Arm mit hämodynamisch kontrollierter medikamentöser Therapie randomisiert. Die Ergebnisse zeigen eine niedrigere Reblutungsrate bei Patienten mit TIPS-Implantation (7\%) verglichen zur medikamentösen Gruppe (26\%). Die Patienten in der TIPS-Gruppe zeigten eine höhere Enzephalopathierate (18\%) verglichen mit der medikamentösen Therapiegruppe (8\%) [322].

Nach dem aktuellem Positionspapier der Baveno VI-Konsensuskonferenz [268] wird die Durchführung eines TIPS mit beschichtetem Stent bei Versagen der first-line-Therapie empfoh- len. Dies gilt auch für eine Rezidivblutung innerhalb der ersten 5 Tage nach endoskopischer Therapie.

\section{Wann sollten röntgengestützte Interventionen erfol- gen? Welche röntgengestützten Interventionsformen gibt es?}

\section{EMPFEHLUNG 85}

Röntgengestützte Verfahren können in der akuten Varizenblutung, dem Primärversagen, dem Sekundärversagen und dem Versagen der Sekundärprophylaxe eingesetzt werden. Offene Empfehlung, starker Konsens.

\section{EMPFEHLUNG 86}

Aufgrund niedrigerer Stentthromboseraten und niedrigerer Reinterventionsraten sollte ein beschichteter Stent verwendet werden.

Offene Empfehlung, starker Konsens (zwei Enthaltungen).

\section{EMPFEHLUNG 87}

Die Möglichkeit der Embolisation von Varizen sollte im Rahmen der TIPS-Implantation überprüft werden.

Empfehlung, starker Konsens.

\section{EMPFEHLUNG 88}

Das Verfahren der BRTO (balloon-occluded retrograde transvenous obliteration) als Alternative zum TIPS kann aufgrund der vorhanden Datenlage noch nicht empfohlen werden. Empfehlung, starker Konsens.

Röntgengestützte Verfahren werden in der akuten Varizenblutung, dem Primärversagen und dem Versagen der Sekundärprophylaxe eingesetzt.

In der Therapie der gastrointestinalen Blutung bei portaler Hypertension ist die TIPS-Implantation das häufigste eingesetzte röntgengestützte Verfahren. In mehreren Studien [323, 324] und einer Metaanalyse [314] konnte gezeigt werden, dass PTFE-beschichtete Stents den bare-metal Stents insbesondere bezüglich Offenheitsraten deutlich überlegen sind. Diese Empfehlung hat auch Eingang in die AASLD-Guidelines gefunden [325].

Die zusätzliche Embolisation von Varizen und Kollateralgefäßen mittels Coils oder Gefäßverschlusssystemen (z. B. Amplatzer Vascular Plugs) zeigte bezüglich der Senkung der Rezidivblutungsrate schon in früheren Studien vielversprechende Ergebnisse [326]. Die Ergebnisse konnten in einer kontrolliert randomisierten Studie bestätigt werden [327]. Neben den niedrigen Rezidivblutungsraten ist die Varizenembolisation zudem den TIPS-Offen- 
heitsraten zuträglich, da offensichtlich günstigere Strömungsverhältnisse im Shunt herrschen.

Die BRTO (balloon-occluded retrograde transvenous obliteration) als Alternative zum TIPS, insbesondere in der Behandlung gastrischer Varizen zeigt vielversprechende Ergebnisse, wie eine kürzlich publizierte Metaanalyse zeigen konnte [328]. Die klinischen Erfolgsraten liegen bei 97,3\%. Allerdings beruhen die Einschätzungen auf einer Auswahl von 24 Studien (23 retrospektive und eine prospektive), sodass für eine Empfehlung weitere prospektive und randomisierte Studien notwendig sind.

\section{Wann sollten chirurgische Interventionen erfolgen? Welche Operationsformen gibt es?}

\section{EMPFEHLUNG 89}

Chirurgische Verfahren sind bei Patienten mit variköser gastrointestinaler Blutung Reserveverfahren. Als Alternativverfahren zur Anlage eines transjugulären intrahepatischen portosystemischen Shunts (TIPS) kann die Durchführung einer Shuntoperation von einem mit dieser Operation erfahrenen Operateur geprüft werden.

Offene Empfehlung, starker Konsens.

Chirurgische Verfahren sind bei Patienten mit gastrointestinaler Blutung bei portaler Hypertension als Reserveverfahren anzusehen. Bedeutung haben chirurgische Verfahren in diesem Zusammenhang bei Versagen der medikamentösen Therapie und endoskopischen Therapieverfahren oder Versagen der Therapie mit einem TIPS bzw. der Unmöglichkeit einer TIPS-Anlage. Aufgrund der relativ selten durchgeführten Operationsform sollte die Shuntoperation allerdings nur von einem mit dieser Operation erfahrenen Operateur durchgeführt werden. Eine randomisierte Multicenterstudie hat bei Patienten im Stadium Child-Pugh A und B mit therapierefraktärer Blutung die Anlage eines TIPS mit der Anlage eines distalen splenorenalen Shunts (DSRS) verglichen [329]. Die Ergebnisse zeigen keine signifikanten Unterschiede in Bezug auf Reblutung (TIPS 10,5\%; DSRS 5,5\%; $p=0,29$ ) oder Enzephalopathierate (TIPS $50 \%$; DSRS $50 \%$ ). Das Überleben nach 2 und 5 Jahren (TIPS 88 und $61 \%$; DSRS 81 und $62 \%$ ) war nicht signifikant unterschiedlich $(p=0,87)$. Die Studie zeigt weiterhin eine signifikant höhere Reinterventionsrate aufgrund von Thrombose und Stenosen in der TIPS-Gruppe (TIPS $82 \%$; DSRS $11 \%$ ) [329]. Diese höhere Interventionsrate in der TIPS-Gruppe muss allerdings im Zusammenhang mit der Verwendung unbeschichteter TIPS-Stents interpretiert werden. Durch die heutzutage mehrheitlich verwendeten beschichteten Stents ist, wie oben bereits erwähnt, von einer deutlich niedrigeren Interventionsrate auszugehen.

\section{Arbeitsgruppe 6: Prophylaxe und post- endoskopisches Management}

\section{Begleittherapie zur interventionellen Therapie bei akuter Varizenblutung}

\section{EMPFEHLUNG 90}

Bei Patienten mit Leberzirrhose sollte bei V. a. eine Varizenblutung bzw. nach endoskopischer Diagnose eine antibiotische Therapie mit Substanzen, welche wirksam gegen gramnegative Enterobacteriaceae sind, für sieben Tage durchgeführt werden.

Empfehlung, starker Konsens.

\section{EMPFEHLUNG 91}

Die Therapie kann mit Ciprofloxazin (400 mg i. v. alle 12 Stunden, alternativ $2 \times 500 \mathrm{mg}$ p. o.), Ceftriaxon ( $2 \mathrm{~g}$ i. v. alle 24 Stunden) oder Norfloxazin (400 mg p. o. alle 12 Stunden) durchgeführt werden. Bei Patienten mit fortgeschrittener Leberzirrhose (Child-Pugh C) und in Zentren mit hoher Chinolon-Resistenz sind Gruppe-3-Cephalosporine zu bevorzugen. Offene Empfehlung, starker Konsens.

\section{EMPFEHLUNG 92}

Eine intravenös begonnene antibiotische Therapie kann ggf. nach 2 - 3 Tagen auf eine orale Applikation umgestellt werden.

Offene Empfehlung, starker Konsens.

Bakterielle Infektionen spielen eine wichtige Rolle in der Pathogenese der Varizenblutung und ca. 20 - 40\% der Patienten, die mit einer akuten Varizenblutung hospitalisiert werden, entwickeln innerhalb von 48 Stunden eine bakterielle Infektion [330, 331]. Eine Rezidivblutung innerhalb der ersten sieben Tage trat bei 43,5\% der Patienten mit bakterieller Infektion auf, im Vergleich zu 9,8\% der Patienten ohne Infektion [330]. Die häufigsten Erreger sind E. coli, Klebsiella spp., Enterococcus und Pseudomonas spp., klinische Manifestationen am häufigsten spontan bakterielle Peritonitiden, Harnwegsinfektionen oder Blutstrominfektionen [332, 333]. Eine frühzeitige antibiotische Therapie mit Chinolonen senkt die Infektionsrate von 37,2 auf 10\% [334] bzw. von 45 auf $10 \%$ [333]. Patienten mit Child-C-Zirrhose haben ein deutlich höheres Infektionsrisiko als Patienten mit Child-A/B-Zirrhose (52,9 vs. 18,2\%) [335]. Eine Cochrane-Metaanalyse von 12 Studien mit insgesamt 1241 Patienten konnte zeigen, dass die Antibiotika-Prophylaxe die Gesamtsterblichkeit (RR 0,79, $95 \%$ KI 0,630,98), die Sterblichkeit durch bakterielle Infektionen (RR 0,43, $95 \%$ KI 0,19-0,97), die Rate an bakteriellen Infektionen (RR 0,35, 
$95 \%$ KI 0,26-0,47) sowie die Rezidivblutungsrate (RR 0,53, $95 \%$ KI 0,38-0,74) signifikant senken kann [73]. Da etwa die Hälfte der bakteriellen Infektionen bereits in den ersten 24 Stunden auftritt, sollte die Antibiotika-Prophylaxe frühzeitig bei vermuteter Varizenblutung begonnen werden. Eine Studie legt nahe, dass ein Therapiefenster von 8 Stunden nicht überschritten werden sollte [336]. Die ersten Studien wurden mit oral oder parenteral verabreichten Chinolonen durchgeführt. Eine jüngere Studie [74] zeigte eine höhere Wirksamkeit von Ceftriaxon (Infektionsrate $2 \%$ mit Ceftriaxon vs. $12 \%$ mit Norfloxazin); aufgrund der Zunahme der Chinolon-Resistenz bei gramnegativen Bakterien erscheint es daher bedeutsam, lokale Resistenzstatistiken in der Auswahl der Antibiotika zu berücksichtigen.

\section{Medikamentöse Senkung des portalvenösen Drucks}

\section{EMPFEHLUNG 93}

Wenn sich endoskopisch eine Varizenblutung bestätigt hat, soll die bereits eingeleitete Therapie mit vasoaktiven Substanzen fortgesetzt werden.

Starke Empfehlung, starker Konsens.

\section{AUSSAGE 1}

Terlipressin, Somatostatin und Octreotid sind vergleichbar wirksam.

Empfehlung, starker Konsens.

\section{EMPFEHLUNG 94}

Die Therapie sollte bis zu fünf Tage fortgeführt werden. Ein früheres Absetzen der Therapie, aber frühestens 24 Stunden nach Erreichen der klinischen Stabilität, kann bei fehlenden Risikofaktoren erwogen werden. Empfehlung, starker Konsens.

Sowohl das synthetische Vasopressin-Analogon Terlipressin als auch Somatostatin und sein Analogon Octreotid senken den portalvenösen Druck. Terlipressin (2 mg i. v. alle 4 Stunden), Somatostatin $(250 \mu \mathrm{g}$ Bolus gefolgt von $250 \mu \mathrm{g} /$ Stunde Dauerinfusion) und Octreotid $(50 \mu \mathrm{g}$ Bolus gefolgt von $50 \mu \mathrm{g} /$ Stunde Dauerinfusion) sind vergleichbar wirksam, aber auch andere Dosen sind untersucht worden ( $\triangleright$ Tab. 4), sodass in bisherigen Leitlinien für Terlipressin Dosen von 1 - 2 mg als intravenöser Bolus alle 4-6 Stunden aufgeführt werden (Beginn noch vor der Endoskopie) [2]. In einer Cochrane- Metaanalyse von 20 Studien an insgesamt 1609 Patienten konnte erstmals gezeigt werden, dass die Gesamtmortalität durch Terlipressin im Vergleich zu Plazebo signifikant gesenkt wird (RR 0,66, 95 \% KI 0,49-0,88) [337]. Eine erneute Metaanalyse 2012, in der 30 Studien mit insgesamt 3111 Patienten ausgewertet wurden, die mit Terlipressin, Somatostatin oder Octreotid behandelt worden waren, zeigte unabhängig von der eingesetzten Substanz eine signifikante Senkung der 7-Tages-Mortalität (RR 0,66, 95 \% KI 0,49-0,88). Unterschiede zwischen Terlipressin, Somatostatin und Octreotid zeigten sich bei allerdings niedriger Evidenzqualität nicht. Die aktuellsten Daten stammen aus einer großen randomisierten Studie an 780 Patienten [72], welche keine signifikanten Unterschiede zwischen den drei Wirkstoffen Terlipressin, Somatostatin und Octreotid hinsichtlich Therapieerfolg an Tag 5 (86,2, 83,4, bzw. 83,8\%; $\mathrm{p}=0,636)$, Rezidivblutungsrate (3,4, 4,8, bzw. 4,4\%; $\mathrm{p}=0,739)$, oder Mortalität $(8,0,8,9$, bzw. $8,8 \%$; $p=0,929)$ ergab. Während in den meisten Studien die Therapie über bis zu 5 Tagen durchgeführt wurde, ergab eine jüngere Studie [71], dass eine verkürzte Therapie mit Terlipressin über 24 Stunden nach erfolgreicher endoskopischer Varizenligatur einer 72-stündigen Therapie nicht unterlegen ist. In der Studie waren aber Hochrisikopatienten mit Child-Score $>=12$ sowie Patienten mit Pfortaderthrombose, Fundusvarizen oder HCC ausgeschlossen.

\section{Gerinnungsfaktoren und Thrombozyten}

\section{AUSSAGE 15}

Es existiert keine Evidenz, die den routinemäßigen Einsatz von Gerinnungsfaktoren oder Thrombozyten unterstützt. Empfehlung, starker Konsens.

\section{AUSSAGE 16}

Die INR spiegelt nicht das Ausmaß der plasmatischen Gerinnungsstörung bei Leberzirrhose wider.

Empfehlung, starker Konsens.

\section{AUSSAGE 17}

Der Einsatz von rekombinantem Faktor VIla und Tranexamsäure wird von der aktuellen Datenlage nicht unterstützt. Empfehlung, starker Konsens.

Patienten mit fortgeschrittener Leberzirrhose haben einen Mangel an Gerinnungsfaktoren mit entsprechender Einschränkung der Blutgerinnungstests, insbesondere der INR. Allerdings fehlen im Gegensatz zu Patienten, die mit Vitamin-K-Antagonisten behandelt werden, auch gerinnungshemmende Proteine wie Antithrombin III oder Plasminogen. Zudem sind Faktor VIII und von-Willebrand-Faktor häufig erhöht [338, 339]. Die klinische Gerinnungsstörung bzw. Blutungsneigung ist daher anhand der klassischen Gerinnungstests nicht verlässlich einzuschätzen und wird in der Regel überschätzt. Die INR kann durch Gabe von Frischplasma (FFP), Prothrombinkomplex-Konzentrat (PPSB) oder rekombinantem Faktor VIla normalisiert werden. 
Der Nutzen von rekombinantem Faktor VIla wurde in zwei randomisierten Studien untersucht [340, 341]. In der ersten Studie fand sich bei Patienten mit Child-B und C- Zirrhose eine höhere Rate an Therapieerfolg, ohne dass eine signifikante Senkung der 5- oder 42-Tage-Mortalität erreicht wurde. Die zweite Studie fokussierte auf Patienten mit Child-B und C-Zirrhose und fand keine signifikanten Unterschiede in den primären Endpunkten (Blutungskontrolle nach 24 Stunden, Rezidivblutungsrate oder Tod innerhalb von 5 Tagen). Beim sekundären Endpunkt 42-Tages-Mortalität war jedoch ein signifikanter Vorteil für die Patienten nachweisbar, die rekombinanten Faktor VIla erhalten hatten (OR 0,31, $95 \%$ KI 0,13-0,74). Eine Metaanalyse der beiden Studien [342] ergab, dass in der Subgruppe von Patienten mit fortgeschrittener Zirrhose (Child-Pugh > 8 Punkte) und aktiver Blutung zum Zeitpunkt der Endoskopie eine signifikante Reduktion von initialem Therapieversagen sowie Rezidivblutungsrate und Tod bis Tag 5 erreicht wurde. Zum Tag 42 ergaben sich aber keine signifikanten Unterschiede mehr. Darüber hinaus erlitten fünf Patienten in der Therapiegruppe eine thromboembolische Komplikation im Vergleich zu keinem Patienten in der Plazebo-Gruppe. In Anbetracht der hohen Potenz von rekombinantem Faktor VIla für die Normalisierung der INR ist es unwahrscheinlich, dass die Gabe von FFP oder PPSB einen signifikanten Langzeitnutzen für Patienten mit Varizenblutung erbringen.

Aussagekräftige Studien zum Nutzen von Thrombozytenkonzentraten liegen nicht vor, in retrospektiven Fallsammlungen erhielten aber 14 - 24,6 \% der Patienten mit Varizenblutung Thrombozytenkonzentrate [343, 344].

\section{Protonenpumpeninhibitoren}

\section{AUSSAGE 18}

Hochdosierte Protonenpumpeninhibitoren (PPI) beeinflussen nicht die Rezidivblutungsrate bei akuter Varizenblutung.

Empfehlung, starker Konsens.

\section{EMPFEHLUNG 95}

Protonenpumpeninhibitoren sollen zur Prophylaxe der Rezidivblutung von Varizen nicht eingesetzt werden, sondern nur dann, wenn andere Indikationen dies erfordern.

Starke Empfehlung, Starker Konsens.

Eine Metaanalyse von 20 Studien [345] ergibt keinen Vorteil einer hochdosierten PPI-Therapie in einem klinisch relevanten Endpunkt. Einzig die Größe der Ligaturulzera lässt sich signifikant vermindern, dem steht aber ein erhöhtes Risiko für eine spontan bakterielle Peritonitis gegenüber.

\section{Prävention der hepatischen Enzephalopathie}

\section{EMPFEHLUNG 96}

Lactulose p. o. kann das Risiko einer hepatischen Enzephalopathie vermindern, alternativ kann orales Rifaximin eingesetzt werden.

Offene Empfehlung, starker Konsens.

In einer Studie an 70 Patienten mit akuter Varizenblutung ließ sich die Rate an hepatischer Enzephalopathie von 40 auf 14\% senken $(p=0,03)$ [346]. Eine Studie verglich die Wirksamkeit von Lactulose im Vergleich zu Rifaximin und fand keine Unterschiede in der Rate der hepatischen Enzephalopathie oder Tod (10/60 vs. 9/60 bzw. 8/60 vs. 9/60, p jeweils 1,0$)$ [347].

\section{Wie sollte die Sekundärprophylaxe nach Ösophagus- varizenligatur oder Fundus-Varizeninjektion durchge- führt werden?}

\section{EMPFEHLUNG 97}

Zur Sekundärprophylaxe der Ösophagusvarizenblutung soll eine Kombinationstherapie aus nicht-selektiver Betablockade und Gummibandligatur durchgeführt werden.

Starke Empfehlung, starker Konsens.

\section{EMPFEHLUNG 98}

Kann eine Kombinationstherapie nicht erfolgen, soll eine Monotherapie mit einem nicht-selektiven Betablocker oder die Gummibandligatur durchgeführt oder ein TIPS angelegt werden.

Starke Empfehlung, Konsens.

\section{AUSSAGE 19}

Carvedilol stellt eine wirksame Alternative zu den nicht-selektiven Betablockern dar.

Empfehlung, starker Konsens.

\section{EMPFEHLUNG 99}

Bei der Sekundärprophylaxe der Fundusvarizenblutung sollte grundsätzlich analog zur Ösophagusvarizenblutung verfahren werden. Es soll jedoch anstelle der Gummibandligatur die Injektionstherapie mit Cyanoacrylat als endoskopisches Verfahren eingesetzt werden.

Empfehlung/starke Empfehlung, starker Konsens. 


\section{EMPFEHLUNG 100}

In der Sekundärprophylaxe der Magenfundusvarizenblutung kann alternativ ein TIPS angelegt werden.

Offene Empfehlung, Konsens.

Für Patienten, die eine Varizenblutung überlebt haben, beträgt das Risiko einer Rezidivblutung bis zu 60 \% bei einer Mortalitätsrate von bis zu 33 \% [348]. Das Verhindern einer erneuten Blutung nach überstandener Initialblutung ist somit von zentraler Bedeutung.

Verschiedene Methoden zur Sekundärprophylaxe, die z. T. auch kombiniert werden können, stehen zur Verfügung. Medikamentös stehen nicht-selektive Betablocker (NSBB, in Deutschland ist nur noch Propranolol in Gebrauch) und Carvedilol, allein oder in Kombination mit Nitraten (Isosorbitmononitrat, ISMN) zur Verfügung. Die Sklerotherapie der Ösophagusvarizen ist von der Gummibandligatur (EVL) verdrängt worden und spielt praktisch keine Rolle mehr. Bei Fundusvarizen kommt in der Regel eine Injektion mit n-Butyl-2-Cyanoacrylat (z. B. Histoacryl ${ }^{\circledR}$ ) zur Anwendung. Weitere Möglichkeiten stellen der TIPS sowie der chirurgische Shunt dar.

Eine Metaanalyse aus 12 Studien hat die nicht-selektiven Betablocker Propranolol sowie Nadolol [349] mit keiner aktiven Behandlung verglichen. Es fand sich eine Reduktion des Blutungsrisikos, die Mortalität hingegen wurde nicht verringert [350].

Durch die Kombination von NSBB mit ISMN kann die Rezidivblutungsrate noch weiter gesenkt werden [351]. Eine Reduktion der Mortalität wird nicht erreicht [352]. Die Rate unerwünschter Wirkungen steigt unter der Kombinationstherapie allerdings.

In einer neueren Studie wurde Carvedilol mit der Kombination aus NSBB und ISMN verglichen [353]. Die Effektivität war ähnlich bei weniger unerwünschten Wirkungen durch Carvedilol.

Eine aktuelle Studie verglich die Kombination aus Simvastatin, EVL und NSBB mit Plazebo, EVL und NSBB [354]. Hierbei fand sich kein Unterschied in der Rezidivblutungsrate, allerdings eine geringere Mortalität bei den Patienten mit einer erhaltenen Leberfunktion in der Simvastatin-Gruppe.

Der Vergleich von EVL mit NSBB in Kombination mit ISMN zeigt uneinheitliche Ergebnisse. Eine Einzelstudie zeigte eine geringere Rezidivblutungsrate unter VBL verglichen mit NSBB und ISMN [355], fand im Follow-up aber trotz der niedrigeren Rezidivblutungsrate eine höhere Mortalität in der EVL-Gruppe [356]. Eine Metaanalyse aus sechs Studien zeigte keinen Unterschied für die Rezidivblutung zwischen den beiden Behandlungsmodalitäten. Allerdings fand sich eine höhere Gesamtmortalität bei den Patienten in der EVL-Gruppe [357]. Drei weitere Metaanalysen, die die medikamentöse Behandlung (entweder NSBB allein oder in Kombination mit ISMN) mit EVL verglichen, fanden weder einen Unterschied in der Rezidivblutungsrate noch in der Mortalität [358 360]. In einer kleinen Studie wurde Carvedilol mit EVL verglichen. Während sich für die Rezidivblutung kein Unterschied zwischen den beiden Verfahren ergab, fand sich für Carvedilol ein Trend zu einer geringeren Mortalität [361].
In einer großen Zahl von Studien wurde die Kombination aus medikamentöser Therapie und EVL untersucht. Auch hier waren die Ergebnisse uneinheitlich. Eine Metaanalyse fand keinen Unterschied in der Rezidivblutungsrate zwischen einer Kombinationstherapie aus Medikamenten und EVL und den jeweiligen Behandlungsmodalitäten allein [358]. Eine weitere Metaanalyse konnte für die Kombinationstherapie eine Reduktion der Rezidivblutungsrate, nicht jedoch der Mortalität zeigen [360]. In einer weiteren Metaanalyse wurde die Kombination aus EVL und medikamentöser Therapie gegen EVL (sieben Studien) bzw. gegen die medikamentöse Therapie (drei Studien) untersucht [362]. Die Kombinationstherapie zeigte eine Reduktion der Rezidivblutungsrate verglichen mit den anderen Modalitäten. Gegenüber EVL allein fand sich außerdem auch eine niedrigere Mortalität. In der aktuellsten Metaanalyse, die die Kombinationstherapie mit EVL oder medikamentöser Therapie allein verglich [363], zeigte sich bei den Studien, die EVL mit der Kombinationstherapie verglichen, eine niedrigere Rezidivblutungsrate in dem Kombinationsarm, während sich bei den Studien, bei denen die EVL zur medikamentösen Therapie hinzugefügt wurde, weder ein Unterschied im Hinblick auf die Rezidivblutungsrate noch auf die Mortalität zeigte.

Diskutiert wird, ob durch NSBB bei Patienten mit Leberzirrhose und therapierefraktärem Aszites die Mortalitätsrate ansteigt. Während zwei Arbeitsgruppen [290, 364] eine höhere Mortalität bei Patienten mit therapierefraktärem Aszites bzw. mit spontan bakterieller Peritonitis nachweisen konnten, kommen zwei neuere Studien [291, 292] bei Patienten mit Aszites auf der Transplantationsliste bzw. Patienten mit akut-auf-chronischem Leberversagen zu dem Ergebnis, dass durch die NSBB-Therapie die Mortalität im Gegenteil sinkt. Dementsprechend kann eine abschließende Empfehlung für oder gegen eine Therapie mit NSBB bei therapierefraktärem Aszites nicht gegeben werden.

Die Datenlage für die Sekundärprophylaxe nach Fundusvarizenblutung ist dünn. In einer Studie wurden NSBB mit der n-Butyl-2-Cyanoacrylat-Injektion verglichen [365]. Dabei zeigten sich bei Patienten, die mittels Injektion behandelt wurden, eine niedrigere Rezidivblutungsrate und eine niedrigere Mortalität.

Die Insertion eines TIPS zeigte in drei Metaanalysen eine Überlegenheit gegenüber der Sklerotherapie oder der Varizenligatur in der Sekundärprophylaxe der Blutung [320, 321, 366]. Dabei zeigte sich kein Unterschied in der Mortalität, allerdings eine höhere Inzidenz der hepatischen Enzephalopathie bei den mit einem TIPS behandelten Patienten.

Eine aktuelle Studie verglich die Anlage eines beschichteten TIPS mit relativ kleinem Durchmesser $(8 \mathrm{~mm})$ mit NSBB und ISMN [322]. Hierbei war der TIPS in der Prophylaxe der Rezidivblutung effektiver als die medikamentöse Therapie, beim Gesamtüberleben ergab sich allerdings kein Unterschied.

Die Shuntchirurgie spielt in der Sekundärprophylaxe praktisch keine Rolle mehr. Sie kommt nur noch bei Patienten zum Einsatz, bei denen Kontraindikationen für eine TIPS-Anlage (z. B. Pfortaderthrombose) bestehen. Entsprechend dünn ist die Studienlage. Eine Metaanalyse, die verschiedene Shuntverfahren (TIPS und chirurgische Shunts) mit der endoskopischen Behandlung verglich, konnte für alle Shuntverfahren eine ähnliche Effektivität im Hinblick auf eine Rezidivblutung zeigen [367]. Die operative Unterbindung bzw. Ligatur von Kollateralen aus dem Pfortader- 
stromgebiet zur Senkung des Varizendruckes (Sperroperation) kommt heutzutage nur noch in wenigen Einzelfällen zur Anwendung. Weiterhin kann bei therapierefraktären Varizenblutungen die Katheterembolisation der Milzarterien erwogen werden.

\section{Ab wann kann die Antikoagulation/Thrombozytenag- gregations-hemmung wiederaufgenommen werden?}

\section{EMPFEHLUNG 101}

Die Fortführung oder Wiederaufnahme der antithrombotischen Medikation sollte vom individuellen Blutungs- und Thromboembolierisiko des Patienten abhängig gemacht werden.

Empfehlung, starker Konsens.

\section{EMPFEHLUNG 102}

- Tab. 8 Zusammenfassung der Empfehlung zur Wiederaufnahme der OAK/TZA.

\begin{tabular}{|c|c|}
\hline $\begin{array}{l}\text { frühe Wiederaufnahme der } \\
\text { OAK/TZA }\end{array}$ & $\begin{array}{l}\text { spätere Wiederaufnahme der } \\
\text { OAK/TZA }\end{array}$ \\
\hline \multicolumn{2}{|l|}{ Ischämie } \\
\hline $\begin{array}{l}\text { mechanischer Herzklappenersatz } \\
\text { (insb. in Mitralis-Position) }\end{array}$ & $\begin{array}{l}\text { stattgehabte (nicht akute, mind. } \\
6 \text { Wochen alte) tiefe Beinvenen- } \\
\text { thrombose/Lungenembolie } \\
\text { (Schweregrad I-II) }\end{array}$ \\
\hline \multicolumn{2}{|l|}{$\begin{array}{l}\text { Z. n. komplexer PCI (langstreckige } \\
\text { Intervention, Intervention des } \\
\text { Hauptstamms) }\end{array}$} \\
\hline Z. n. $\mathrm{PCl} \leq 4$ Wochen & Z. n. $\mathrm{PCl}>4$ Wochen \\
\hline $\begin{array}{l}\text { Hoher CHA2DS2-VASc-Score } \\
\text { (>2 Punkte) }\end{array}$ & $\begin{array}{l}\text { niedriger CHA2DS2-VASC-Score } \\
\text { ( } \leq 2 \text { Punkte) }\end{array}$ \\
\hline Z.n. PCI bei ACS & $\begin{array}{l}\text { Z. n. elektiver } \mathrm{PCl} \text { bei stabiler } \\
\text { Angina pectoris }\end{array}$ \\
\hline \multicolumn{2}{|l|}{$\begin{array}{l}\text { Z. n. Implantation von bioresor- } \\
\text { bierbaren Stents (Absorb) }\end{array}$} \\
\hline \multicolumn{2}{|l|}{ Z. n. Stentthrombose } \\
\hline \multicolumn{2}{|l|}{ Blutungen } \\
\hline $\begin{array}{l}\text { suffizient versorgte gastrointes- } \\
\text { tinale Blutung }\end{array}$ & Ösophagusvarizenblutung \\
\hline $\begin{array}{l}\text { niedriges Risiko für Rezidivblu- } \\
\text { tung (Forrest IIc, III) }\end{array}$ & $\begin{array}{l}\text { hohes Risiko für Rezidivblutungen } \\
\text { (Forrest la, Ib, Ila, Ilb) }\end{array}$ \\
\hline $\begin{array}{l}\text { Prädiktoren für ein generell nied- } \\
\text { riges Blutungsrisiko (junges Alter, } \\
\text { männliches Geschlecht, hoher } \\
\text { BMI) }\end{array}$ & $\begin{array}{l}\text { Prädiktoren für ein generell } \\
\text { erhöhtes Blutungsrisiko (hohes } \\
\text { Alter, weibliches Geschlecht, } \\
\text { niedriger BMI) }\end{array}$ \\
\hline
\end{tabular}

Bei gastrointestinalen Blutungen muss in vielen Fällen eine orale Antikoagulation und/oder Thrombozytenaggregationshemmung pausiert werden. Der Zeitpunkt der Wiederaufnahme der Medikamente richtet sich zum einen nach dem Risiko für ischämische Komplikationen unter der Pausierung der antithrombozytären Therapie und zum anderen nach dem Risiko für (Rezidiv)-Blutungen nach Wiederaufnahme der Medikation. Im klinischen Alltag wird die Entscheidung für die Wiederaufnahme der Medikamente zumeist sehr individuell getroffen. Große klinisch-randomisierte Studien, die verschiedene Therapiekonzepte (z. B. frühe vs. späte Wiederaufnahme der Medikation) miteinander verglichen haben, fehlen. Es gibt aber sowohl aufseiten des Blutungsrisikos als auch aufseiten des Thromboserisikos Determinanten, die für eine frühe bzw. für eine spätere Wiederaufnahme der Medikation sprechen ( $\triangleright$ Tab. 8 in der Empfehlung 108). Eine frühe Wiederaufnahme der antithrombozytären Medikation wird bei Patienten mit hohem Thromboserisiko und niedrigem Blutungsrisiko favorisiert, während eine spätere Wiederaufnahme der Medikation bei Patienten mit niedrigem Thromboserisiko und hohem Blutungsrisiko zu empfehlen ist.

Bei KHK-Patienten nach Koronarintervention bedingen die klinischen Begleitumstände (akutes Koronarsyndrom [ACS] vs. elektive perkutane koronare Intervention $[\mathrm{PCI}]$ ) und der zeitliche Abstand zur Koronarintervention die Notwendigkeit und den Zeitpunkt der Wiederaufnahme der Thrombozytenaggregationshemmung [368]. Generell gilt hier, dass nach PCI bei einem ACS die Empfehlung für eine 12-monatige duale Plättchenhemmung besteht [1]. Nach elektiver PCI bei stabiler Angina pectoris besteht die Indikation für eine duale Plättchenhemmung je nach Art des verwendeten Stents für 1 - 6 Monate [369]. Kommt es unter einer dualen Plättchenhemmung zu einer gastrointestinalen Blutung mit ggf. Notwendigkeit zur endoskopischen Versorgung, so wird im klinischen Alltag zumeist die Therapie mit ASS fortgeführt, und die Therapie mit dem P2Y12-Rezeptorhemmer (Clopidogrel, Prasugrel, Ticagrelor) wird pausiert. Tab. 8 fasst Faktoren zusammen, die für eine frühe vs. späte Re-Initiierung einer dualen Plättchenhemmung sprechen. Bei KHK-Patienten ohne Koronarintervention in jüngster Vergangenheit besteht in der Regel eine Monotherapie mit ASS. Je nach Schweregrad der Blutung muss diese Therapie für eine endoskopische Versorgung der Blutung pausiert werden. Auch hier gelten dieselben Determinanten wie bei dualer Plättchenhemmung, die eine frühe bzw. eine spätere Re-Initiierung der Therapie favorisieren.

\section{Wann ist eine second-look-Endoskopie indiziert?}

\section{EMPFEHLUNG 103}

Eine früh elektive Kontrollendoskopie (innerhalb von $72 \mathrm{~h}$ nach gastrointestinaler Blutung) soll nicht regelhaft durchgeführt werden.

Starke Empfehlung, starker Konsens.

Sie kann im Einzelfall bei Risikofaktoren erwogen werden.

Offene Empfehlung, starker Konsens. 
Der Einsatz einer second-look-Endoskopie nach gastrointestinaler Blutung ist sehr zurückhaltend zu stellen. Die ASGE-Leitlinie beispielsweise erwähnt ausdrücklich, dass eine routinemäßige, geplante second-look-Endoskopie innerhalb von $24 \mathrm{~h}$ nach einer (oberen) gastrointestinalen Blutung nicht empfohlen wird [81, 370]. Dieser Empfehlung folgt auch die europäische Fachgesellschaft, die aber bei „klinischen Hinweisen auf eine erneute Blutung eine Re-Endoskopie vorsieht“ [1], bevor zu weiteren Maßnahmen der Blutstillung gegriffen werden soll. Eine solche Endoskopie ist dann aber nicht mehr als „elektive“ oder „routinemäßige“ second-look-Endoskopie zu bezeichnen.

In der Literatur wurde die Wertigkeit einer second-look-Endoskopie nach gastrointestinaler Blutung insbesondere bei Blutungen aus peptischen Magenulzera untersucht. Zeigt eine Metaanalyse mit kleiner Fallzahl noch eine signifikante Reduktion von Blutungsrezidiven und Notfalloperationen durch die second-look-Endoskopie [371], so verliert sich dieser Effekt, wenn - der heute gültige Standard - eine hochdosierte Protonenpumpenhemmer-Behandlung durchgeführt wird [372]. Zusammengefasst erscheint der Einsatz der second-look-Endoskopie aus medizinischer, aber auch sozioökonomischer Sicht nicht mehr zeitgemäß [371, 373]. Allenfalls in einer noch genau zu definierenden Risikopopulation, also beispielsweise Patienten mit hämodynamischer Instabilität bei aktiver Blutung in der Index-Endoskopie oder sehr großen Ulzera, wäre eine second-look-Maßnahme zu diskutieren.

Wissenschaftliche Daten zur Effektivität von second-look-Endoskopien bei unteren gastrointestinalen Blutungen liegen nicht vor, finden auch keine Beachtung in den vorliegenden Leitlinien [21] und sind somit zu vermeiden.

Erwähnung finden soll noch das Vorgehen bei Varizenblutungen: Bei fehlender Blutstillung einer aktiven Varizenblutung durch die Endoskopie wird nach Ballontamponade eine Re-Endoskopie nach spätestens $24 \mathrm{~h}$ empfohlen [374]. Eine solche Endoskopie ist aber im Rahmen der primären Blutstillung zu sehen, und nicht als „elektive“ second-look-Maßnahme zu betrachten.

\section{Überwachung von Patienten nach endoskopischen Untersuchungen}

Die postinterventionelle Überwachung richtet sich nach Sedierung und Schwere bzw. endoskopischem Therapieerfolg der gastrointestinalen Blutung.

In der S3-Leitlinie Sedierung in der gastrointestinalen Endoskopie [375] wird empfohlen, die postinterventionelle Überwachung an die Auswahl des Sedativums und dessen Wirkdauer und Halbwertszeit und dem zu erwartenden Patientenrisiko [376] anzupassen. Die Überwachung hat lückenlos, von geschultem Fachpersonal und in einem separaten Aufwachbereich stattzufinden. Hier sollte gemäß anästhesiologischen Leitlinien [377] ein Monitoring des Patienten (Pulsoxymetrie, Blutdruck, ggf. EKG) stattfinden und entsprechende Vorsichtsmaßnahmen (wie Medikamente, Sauerstoffanschluss, Absaugung und Hilfsmittel für Reanimation) verfügbar sein. Als Parameter für die Entlassung aus dem Aufwachbereich werden der Wachheitsgrad, die Orientiertheit und die Vitalparameter des Patienten angegeben [377]. Zur Abschätzung der Entlassungsfähigkeit des Patienten werden z. T. ScoringSysteme wie das Post Anaesthetic Discharge Scoring System, kurz
„PADSS“ empfohlen und eingesetzt [378, 379]. Hierbei werden Vitalzeichen, Mobilität, Übelkeit/Erbrechen, Schmerzen, Blutung und Miktion überprüft und klassifiziert.

Neben der reinen Risikoabschätzung durch die Vorerkrankungen des Patienten, die ASA-Klassifikation und die Auswahl des Sedativums sollte sich die postinterventionelle Überwachung auch an der Schwere der gastrointestinalen Blutung und dem Therapieerfolg durch die endoskopische Maßnahme orientieren. Besteht einerseits bei einer aktiven Varizenblutung die Indikation zur weiteren intensivmedizinischen Überwachung mit allen damit einhergehenden Konsequenzen und Möglichkeiten, so kann andererseits ein mobiler und „Hämoglobin-stabiler“ Patient nach endoskopischem Ausschluss einer oberen gastrointestinalen Blutung ggf. wieder entlassen werden.

\section{Interessenkonflikt}

Die Autoren geben an, dass kein Interessenkonflikt besteht.

Literatur

[1] Gralnek IM, Dumonceau JM, Kuipers EJ et al. Diagnosis and management of nonvariceal upper gastrointestinal hemorrhage: European Society of Gastrointestinal Endoscopy (ESGE) Guideline. Endoscopy 2015; 47: a1 a46

[2] Denzer U, Beilenhoff U, Eickhoff A et al. S2k guideline: quality requirements for gastrointestinal endoscopy, AWMF registry no. 021-022. Zeitschrift fur Gastroenterologie 2015; 53: 1496 - 1530

[3] Wacharasint P, Nakada TA, Boyd JH et al. Normal-range blood lactate concentration in septic shock is prognostic and predictive. Shock 2012; 38: $4-10$

[4] Nguyen HB, Rivers EP, Knoblich BP et al. Early lactate clearance is associated with improved outcome in severe sepsis and septic shock. Crit Care Med 2004; 32: 1637-1642

[5] Koch A, Buendgens L, Duckers $\mathrm{H}$ et al. Bleeding origin, patient-related risk factors, and prognostic indicators in patients with acute gastrointestinal hemorrhages requiring intensive care treatment. A retrospective analysis from 1999 to 2010. Medizinische Klinik, Intensivmedizin und Notfallmedizin 2013; 108: 214-222

[6] Rockey DC, Elliott A, Lyles T. Prediction of esophageal varices and variceal hemorrhage in patients with acute upper gastrointestinal bleeding. Journal of investigative medicine: the official publication of the American Federation for Clinical Research 2016; 64: 745-751

[7] Castera L, Pinzani M, Bosch J. Non invasive evaluation of portal hypertension using transient elastography. Journal of hepatology 2012; 56: $696-703$

[8] Salzl P, Reiberger T, Ferlitsch M et al. Evaluation of portal hypertension and varices by acoustic radiation force impulse imaging of the liver compared to transient elastography and AST to platelet ratio index. Ultraschall in Med 2014; 35: 528-533

[9] Attia D, Schoenemeier B, Rodt T et al. Evaluation of Liver and Spleen Stiffness with Acoustic Radiation Force Impulse Quantification Elastography for Diagnosing Clinically Significant Portal Hypertension. Ultraschall in Med 2015; 36: 603-610

[10] Takuma Y, Nouso K, Morimoto Y et al. Prediction of oesophageal variceal bleeding by measuring spleen stiffness in patients with liver cirrhosis. Gut 2016; 65: 354-355

[11] Blatchford O, Murray WR, Blatchford M. A risk score to predict need for treatment for upper-gastrointestinal haemorrhage. Lancet 2000; 356: $1318-1321$ 
[12] Cheng DW, Lu YW, Teller T et al. A modified Glasgow Blatchford Score improves risk stratification in upper gastrointestinal bleed: a prospective comparison of scoring systems. Alimentary pharmacology \& therapeutics 2012; 36: $782-789$

[13] Stanley AJ. Update on risk scoring systems for patients with upper gastrointestinal haemorrhage. World journal of gastroenterology: WJG 2012; 18: $2739-2744$

[14] Rockall TA, Logan RF, Devlin HB et al. Risk assessment after acute upper gastrointestinal haemorrhage. Gut 1996; 38: 316-321

[15] Dicu D, Pop F, lonescu D et al. Comparison of risk scoring systems in predicting clinical outcome at upper gastrointestinal bleeding patients in an emergency unit. Am J Emerg Med 2013; 31: 94 - 99

[16] de Franchis R, Baveno VIF. Expanding consensus in portal hypertension: Report of the Baveno VI Consensus Workshop: Stratifying risk and individualizing care for portal hypertension. Journal of hepatology 2015; 63: $743-752$

[17] Laine L. Risk Assessment Tools for Gastrointestinal Bleeding. Clinical gastroenterology and hepatology: the official clinical practice journal of the American Gastroenterological Association 2016; 14: 1571 - 1573

[18] Aoki T, Nagata N, Shimbo T et al. Development and Validation of a Risk Scoring System for Severe Acute Lower Gastrointestinal Bleeding. Clinical gastroenterology and hepatology: the official clinical practice journal of the American Gastroenterological Association 2016; 14: 1562 - 1570 e1562

[19] Camus M, Jensen DM, Ohning GV et al. Comparison of Three Risk Scores to Predict Outcomes of Severe Lower Gastrointestinal Bleeding. Journal of clinical gastroenterology 2016; 50: 52 - 58

[20] Patel R, Clancy R, Crowther E et al. A rectal bleeding algorithm can successfully reduce emergency admissions. Colorectal disease: the official journal of the Association of Coloproctology of Great Britain and Ireland 2014; 16: $377-381$

[21] Pasha SF, Shergill A, Committee ASoP et al. The role of endoscopy in the patient with lower GI bleeding. Gastrointestinal endoscopy 2014; 79: $875-885$

[22] Lin HJ, Wang K, Perng CL et al. Early or delayed endoscopy for patients with peptic ulcer bleeding. A prospective randomized study. Journal of clinical gastroenterology 1996; 22: $267-271$

[23] Lim LG, Ho KY, Chan YH et al. Urgent endoscopy is associated with lower mortality in high-risk but not low-risk nonvariceal upper gastrointestinal bleeding. Endoscopy 2011; 43: 300-306

[24] Robertson M, Majumdar A, Boyapati R et al. Risk stratification in acute upper Gl bleeding: comparison of the AIMS65 score with the GlasgowBlatchford and Rockall scoring systems. Gastrointestinal endoscopy 2016; 83: $1151-1160$

[25] Garcia-Tsao G, Bosch J. Management of varices and variceal hemorrhage in cirrhosis. The New England journal of medicine 2010; 362: 823-832

[26] Tripathi D, Stanley AJ, Hayes PC et al. U.K. guidelines on the management of variceal haemorrhage in cirrhotic patients. Gut 2015; 64: $1680-1704$

[27] Villanueva C, Piqueras M, Aracil C et al. A randomized controlled trial comparing ligation and sclerotherapy as emergency endoscopic treatment added to somatostatin in acute variceal bleeding. Journal of hepatology 2006; 45: $560-567$

[28] Bañares R, Albillos A, Rincón D et al. Endoscopic treatment versus endoscopic plus pharmacologic treatment for acute variceal bleeding: a meta-analysis. Hepatology 2002; 35: 609-615

[29] Tripathi D, Stanley AJ, Hayes PC et al. UK guidelines on the management of variceal haemorrhage in cirrhotic patients. Gut 2015; 64: 1680-1704

[30] Carbonell N, Pauwels A, Serfaty L et al. Improved survival after variceal bleeding in patients with cirrhosis over the past two decades. Hepatology 2004; 40: $652-659$
[31] Hreinsson JP, Gumundsson S, Kalaitzakis E et al. Lower gastrointestinal bleeding: incidence, etiology, and outcomes in a population-based setting. European journal of gastroenterology \& hepatology 2013; 25: 37 43

[32] Gomez-Outes A, Suarez-Gea ML, Lecumberri R et al. Direct oral anticoagulants in the treatment of venous thromboembolism, with a focus on patients with pulmonary embolism: an evidence-based review. Vasc Health Risk Manag 2014; 10: 627-639

[33] Gomez-Outes A, Terleira-Fernandez AI, Lecumberri R et al. Direct oral anticoagulants in the treatment of acute venous thromboembolism: a systematic review and meta-analysis. Thrombosis research 2014; 134 : $774-782$

[34] Strate LL. Lower GI bleeding: epidemiology and diagnosis. Gastroenterology clinics of North America 2005; 34: 643-664

[35] Strate LL, Saltzman JR, Ookubo R et al. Validation of a clinical prediction rule for severe acute lower intestinal bleeding. The American journal of gastroenterology 2005; 100: 1821 - 1827

[36] Meier A, Messmann H, Golder SK. Endoscopic management of lower gastrointestinal bleeding. Medizinische Klinik, Intensivmedizin und Notfallmedizin 2015; 110: 515-520

[37] Repaka A, Atkinson MR, Faulx AL et al. Immediate unprepared hydroflush colonoscopy for severe lower GI bleeding: a feasibility study. Gastrointestinal endoscopy 2012; 76: 367 - 373

[38] Braun JP, Kumpf O, Deja M et al. The German quality indicators in intensive care medicine 2013-second edition. Ger Med Sci 2013; 11: Doc09

[39] Wehrmann T, Riphaus A. Sedation with propofol for interventional endoscopic procedures: a risk factor analysis. Scandinavian journal of gastroenterology 2008; 43: 368 - 374

[40] Riphaus A, Wehrmann T, Hausmann J et al. S3-guidelines "sedation in gastrointestinal endoscopy" 2014 (AWMF register no. 021/014). Zeitschrift für Gastroenterologie 2015; 53: E1

[41] Ogasawara N, Mizuno M, Masui R et al. Predictive factors for intractability to endoscopic hemostasis in the treatment of bleeding gastroduodenal peptic ulcers in Japanese patients. Clin Endosc 2014; 47: 162 - 173

[42] Braun G, Messmann H. New therapeutical options for heavy gastrointestinal bleeding. Dtsch Med Wochenschr 2015; 140: 888-891

[43] Garcia-Pagan JC, Caca K, Bureau C et al. Early use of TIPS in patients with cirrhosis and variceal bleeding. The New England journal of medicine 2010; 362: $2370-2379$

[44] Hubmann R, Bodlaj G, Czompo M et al. The use of self-expanding metal stents to treat acute esophageal variceal bleeding. Endoscopy 2006; 38: $896-901$

[45] European Association for the Study of the Liver. Electronic address eee. EASL Clinical Practice Guidelines: Liver transplantation. Journal of hepatology 2016; 64: $433-485$

[46] Baradarian R, Ramdhaney S, Chapalamadugu R et al. Early intensive resuscitation of patients with upper gastrointestinal bleeding decreases mortality. The American journal of gastroenterology 2004; 99: 619622

[47] Kwan I, Bunn F, Chinnock P et al. Timing and volume of fluid administration for patients with bleeding. The Cochrane database of systematic reviews 2014; 3: doi:CD002245

[48] Marx G, Schindler AW, Mosch C et al. Intravascular volume therapy in adults: Guidelines from the Association of the Scientific Medical Societies in Germany. European journal of anaesthesiology 2016. doi:10.1097/EJA.0000000000000447

[49] Gattas DJ, Dan A, Myburgh J et al. Fluid resuscitation with 6 \% hydroxyethyl starch (130/0.4 and 130/0.42) in acutely ill patients: systematic review of effects on mortality and treatment with renal replacement therapy. Intensive Care Med 2013; 39: 558-568

[50] Annane D, Siami S, Jaber S et al. Effects of fluid resuscitation with colloids vs crystalloids on mortality in critically ill patients presenting with 
hypovolemic shock: the CRISTAL randomized trial. JAMA: the journal of the American Medical Association 2013; 310: 1809-1817

[51] Bruells CS, Schindler A, Marx G. The role of colloids in intensive care medicine. Evidence instead of emotions. Medizinische Klinik, Intensivmedizin und Notfallmedizin 2015; 110: 133-137

[52] Dellinger RP, Levy MM, Rhodes A et al. Surviving sepsis campaign: international guidelines for management of severe sepsis and septic shock: 2012. Crit Care Med 2013; 41: 580-637

[53] Beloncle F, Meziani F, Lerolle $N$ et al. Does vasopressor therapy have an indication in hemorrhagic shock? Annals of intensive care 2013; 3: 13

[54] Shah A, Stanworth SJ, McKechnie S. Evidence and triggers for the transfusion of blood and blood products. Anaesthesia 2015; 70 (Suppl. 1): 10-19, e13-15

[55] Villanueva C, Colomo A, Bosch A et al. Transfusion strategies for acute upper gastrointestinal bleeding. The New England journal of medicine 2013; 368: 11-21

[56] Jairath V, Kahan BC, Gray A et al. Restrictive versus liberal blood transfusion for acute upper gastrointestinal bleeding (TRIGGER): a pragmatic, open-label, cluster randomised feasibility trial. Lancet 2015; 386: 137 144

[57] (BÄK). B. Querschnitts-Leitlinie der Bundesärztekammer (BÄK) zur Therapie mit Blutkomponenten und Plasmaderivaten (4. überarbeitete und aktualisierte Auflage 2014). 2014

[58] Hwang JH, Fisher DA, Ben-Menachem T et al. The role of endoscopy in the management of acute non-variceal upper $\mathrm{Gl}$ bleeding. Gastrointestinal endoscopy 2012; 75: 1132-1138

[59] Barkun AN, Bardou M, Martel M et al. Prokinetics in acute upper GI bleeding: a meta-analysis. Gastrointestinal endoscopy 2010; 72: 1138 1145

[60] Sreedharan A, Martin J, Leontiadis Gl et al. Proton pump inhibitor treatment initiated prior to endoscopic diagnosis in upper gastrointestinal bleeding. The Cochrane database of systematic reviews 2010. doi:0.1002/14651858.CD005415.pub3:CD005415

[61] Sachar H, Vaidya K, Laine L. Intermittent vs continuous proton pump inhibitor therapy for high-risk bleeding ulcers: a systematic review and meta-analysis. JAMA internal medicine 2014; 174: 1755- 1762

[62] Tripathi D, Stanley AJ, Hayes PC et al. U.K. guidelines on the management of variceal haemorrhage in cirrhotic patients. Gut 2015; 64: $1680-1704$

[63] Kim KR, Jun CH, Cho KM et al. Can proton pump inhibitors reduce rebleeding following Histoacryl sclerotherapy for gastric variceal hemorrhage? The Korean journal of internal medicine 2015; 30: 593-601

[64] Dultz G, Piiper A, Zeuzem S et al. Proton pump inhibitor treatment is associated with the severity of liver disease and increased mortality in patients with cirrhosis. Alimentary pharmacology \& therapeutics 2015; 41: $459-466$

[65] Min YW, Lim KS, Min BH et al. Proton pump inhibitor use significantly increases the risk of spontaneous bacterial peritonitis in 1965 patients with cirrhosis and ascites: a propensity score matched cohort study. Alimentary pharmacology \& therapeutics 2014; 40: 695-704

[66] D'Amico G, Pagliaro L, Bosch J. The treatment of portal hypertension: a meta-analytic review. Hepatology 1995; 22: $332-354$

[67] Wells M, Chande N, Adams P et al. Meta-analysis: vasoactive medications for the management of acute variceal bleeds. Alimentary pharmacology \& therapeutics 2012; 35: 1267-1278

[68] Villanueva C, Ortiz ], Sabat M et al. Somatostatin alone or combined with emergency sclerotherapy in the treatment of acute esophageal variceal bleeding: a prospective randomized trial. Hepatology 1999; 30: 384 389

[69] Sung J], Chung SC, Yung MY et al. Prospective randomised study of effect of octreotide on rebleeding from oesophageal varices after endoscopic ligation. Lancet 1995; 346: 1666 - 1669
[70] Besson I, Ingrand P, Person B et al. Sclerotherapy with or without octreotide for acute variceal bleeding. The New England journal of medicine 1995; 333: 555- 560

[71] Azam Z, Hamid S, Jafri W et al. Short course adjuvant terlipressin in acute variceal bleeding: a randomized double blind dummy controlled trial. Journal of hepatology 2012; 56: 819-824

[72] Seo YS, Park SY, KIM MY et al. Lack of difference among terlipressin, somatostatin, and octreotide in the control of acute gastroesophageal variceal hemorrhage. Hepatology 2014; 60: 954-963

[73] Chavez-Tapia NC, Barrientos-Gutierrez T, Tellez-Avila F et al. Meta-analysis: antibiotic prophylaxis for cirrhotic patients with upper gastrointestinal bleeding - an updated Cochrane review. Alimentary pharmacology \& therapeutics 2011; 34: 509-518

[74] Fernandez J, Ruiz del Arbol L, Gomez C et al. Norfloxacin vs ceftriaxone in the prophylaxis of infections in patients with advanced cirrhosis and hemorrhage. Gastroenterology 2006; 131: 1049-1056; quiz 1285

[75] Engelmann L, Schmitt DV. Tarragona strategy-appropriate antibiotic therapy in the ICU. Medizinische Klinik, Intensivmedizin und Notfallmedizin 2014; 109: $156-161$

[76] Hearnshaw SA, Logan RF, Lowe D et al. Use of endoscopy for management of acute upper gastrointestinal bleeding in the UK: results of a nationwide audit. Gut 2010; 59: 1022-1029

[77] Bai Y, Guo JF, Li ZS. Meta-analysis: erythromycin before endoscopy for acute upper gastrointestinal bleeding. Alimentary pharmacology \& therapeutics 2011; 34: 166-171

[78] Szary NM, Gupta R, Choudhary A et al. Erythromycin prior to endoscopy in acute upper gastrointestinal bleeding: a meta-analysis. Scandinavian journal of gastroenterology 2011; 46: 920-924

[79] Theivanayagam S, Lim RG, Cobell W] et al. Administration of erythromycin before endoscopy in upper gastrointestinal bleeding: a metaanalysis of randomized controlled trials. Saudi journal of gastroenterology: official journal of the Saudi Gastroenterology Association 2013; 19: $205-210$

[80] Winstead NS, Wilcox CM. Erythromycin prior to endoscopy for acute upper gastrointestinal haemorrhage: a cost-effectiveness analysis. Alimentary pharmacology \& therapeutics 2007; 26: 1371 - 1377

[81] Barkun AN, Bardou M, Kuipers Ej et al. International consensus recommendations on the management of patients with nonvariceal upper gastrointestinal bleeding. Annals of internal medicine 2010; 152: 101 113

[82] Koch DG, Arguedas MR, Fallon MB. Risk of aspiration pneumonia in suspected variceal hemorrhage: the value of prophylactic endotracheal intubation prior to endoscopy. Digestive diseases and sciences 2007; 52: $2225-2228$

[83] Rudolph S], Landsverk BK, Freeman ML. Endotracheal intubation for airway protection during endoscopy for severe upper $\mathrm{Gl}$ hemorrhage. Gastrointestinal endoscopy 2003; 57: 58-61

[84] Rehman A, Iscimen R, Yilmaz M et al. Prophylactic endotracheal intubation in critically ill patients undergoing endoscopy for upper GI hemorrhage. Gastrointestinal endoscopy 2009; 69: e55-e59

[85] Hayat U, Lee PJ, Ullah H et al. Association of prophylactic endotracheal intubation in critically ill patients with upper GI bleeding and cardiopulmonary unplanned events. Gastrointestinal endoscopy 2016. doi:10.1016/j.gie.2016.12.008

[86] Janssens $\mathrm{U}$, Burchardi $\mathrm{H}$, Duttge $\mathrm{G}$ et al. Change in therapy target and therapy limitations in intensive care medicine. Position paper of the Ethics Section of the German Interdisciplinary Association for Intensive Care and Emergency Medicine. Der Anaesthesist 2013; 62: 47-52

[87] Denzer U, Beilenhoff U, Eickhoff A et al. S2k guideline: quality requirements for gastrointestinal endoscopy, AWMF registry no. 021-022, recommendations. Zeitschrift für Gastroenterologie 2015; 53: E1 -E227 
[88] Seshasai SR, Wijesuriya S, Sivakumaran R et al. Effect of aspirin on vascular and nonvascular outcomes: meta-analysis of randomized controlled trials. Arch Intern Med 2012; 172: 209-216

[89] McQuaid KR, Laine L. Systematic review and meta-analysis of adverse events of low-dose aspirin and clopidogrel in randomized controlled trials. The American journal of medicine 2006; 119: 624-638

[90] Hansen ML, Sorensen R, Clausen MT et al. Risk of bleeding with single, dual, or triple therapy with warfarin, aspirin, and clopidogrel in patients with atrial fibrillation. Arch Intern Med 2010; 170: 1433-1441

[91] Lamberts M, Gislason GH, Lip GY et al. Antiplatelet therapy for stable coronary artery disease in atrial fibrillation patients taking an oral anticoagulant: a nationwide cohort study. Circulation 2014; 129: 1577 1585

[92] Lamberts M, Olesen JB, Ruwald MH et al. Bleeding after initiation of multiple antithrombotic drugs, including triple therapy, in atrial fibrillation patients following myocardial infarction and coronary intervention: a nationwide cohort study. Circulation 2012; 126: 1185-1193

[93] Kikkert W], Hassell ME, Delewi R et al. Predictors and prognostic consequence of gastrointestinal bleeding in patients with ST-segment elevation myocardial infarction. Int J Cardiol 2015; 184: 128-134

[94] Cote R, Zhang Y, Hart RG et al. ASA failure: does the combination ASA/ clopidogrel confer better long-term vascular protection? Neurology 2014; 82: $382-389$

[95] Chen Z, Qian J, Chen Y et al. Addition of cilostazol to conventional dual antiplatelet therapy reduces the risk of cardiac events and restenosis after drug-eluting stent implantation: a meta-analysis. J Clin Pharmacol 2013; 53: $532-539$

[96] Guerrouij M, Uppal CS, Alklabi A et al. The clinical impact of bleeding during oral anticoagulant therapy: assessment of morbidity, mortality and post-bleed anticoagulant management. Journal of thrombosis and thrombolysis $2011 ; 31: 419-423$

[97] Holbrook A, Schulman S, Witt DM et al. Evidence-based management of anticoagulant therapy: Antithrombotic Therapy and Prevention of Thrombosis, 9th ed: American College of Chest Physicians EvidenceBased Clinical Practice Guidelines. Chest 2012; 141: e152S-e184S

[98] Rikala M, Kastarinen H, Tiittanen P et al. Natural history of bleeding and characteristics of early bleeders among warfarin initiators - a cohort study in Finland. Clin Epidemiol 2016; 8: 23-35

[99] Caldeira D, Barra M, Ferreira A et al. Systematic review with meta-analysis: the risk of major gastrointestinal bleeding with non-vitamin $\mathrm{K}$ antagonist oral anticoagulants. Alimentary pharmacology \& therapeutics 2015; $42: 1239-1249$

[100] Gallagher AM, van Staa TP, Murray-Thomas T et al. Population-based cohort study of warfarin-treated patients with atrial fibrillation: incidence of cardiovascular and bleeding outcomes. BMJ Open 2014; 4: e003839

[101] Holster IL, Valkhoff VE, Kuipers EJ et al. New oral anticoagulants increase risk for gastrointestinal bleeding: a systematic review and metaanalysis. Gastroenterology 2013; 145: 105-112 e115

[102] Leon MB, Baim DS, Popma JJ et al. A clinical trial comparing three antithrombotic-drug regimens after coronary-artery stenting. Stent Anticoagulation Restenosis Study Investigators. The New England journal of medicine 1998; 339: 1665-1671

[103] Ng FH, Wong SY, Lam KF et al. Gastrointestinal bleeding in patients receiving a combination of aspirin, clopidogrel, and enoxaparin in acute coronary syndrome. The American journal of gastroenterology 2008; 103: $865-871$

[104] Dewilde WJ, Oirbans T, Verheugt FW et al. Use of clopidogrel with or without aspirin in patients taking oral anticoagulant therapy and undergoing percutaneous coronary intervention: an open-label, randomised, controlled trial. Lancet 2013; 381: 1107-1115

[105] Segal JB, Dzik WH, Transfusion Medicine/Hemostasis Clinical Trials N.. Paucity of studies to support that abnormal coagulation test results predict bleeding in the setting of invasive procedures: an evidencebased review. Transfusion 2005; 45: 1413-1425

[106] Davis A, Walsh M, McCarthy P et al. Tranexamic acid without prophylactic factor replacement for prevention of bleeding in hereditary bleeding disorder patients undergoing endoscopy: a pilot study. Haemophilia 2013; 19: 583 - 589

[107] (BÄK). B. Querschnitts-Leitlinien (BÄK) zur Therapie mit Blutkomponenten und Plasmaderivaten. 2014 http://bundesaerztekammerde/ downloadsQLL_Haemotherapie_2014pdf

[108] Srivastava A, Brewer AK, Mauser-Bunschoten EP et al. Guidelines for the management of hemophilia. Haemophilia 2013; 19: e1 -e47

[109] Denzer U, Beilenhoff U, Eickhoff A et al. S2k guideline: quality requirements for gastrointestinal endoscopy, AWMF registry no. 021-022. Zeitschrift für Gastroenterologie 2015; 53: E1 - E227

[110] Tripodi A, Caldwell SH, Hoffman M et al. Review article: the prothrombin time test as a measure of bleeding risk and prognosis in liver disease. Alimentary pharmacology \& therapeutics 2007; 26: 141 - 148

[111] Tripodi A, Mannucci PM. Abnormalities of hemostasis in chronic liver disease: reappraisal of their clinical significance and need for clinical and laboratory research. Journal of hepatology 2007; 46: 727 - 733

[112] Tripodi A, Anstee QM, Sogaard KK et al. Hypercoagulability in cirrhosis: causes and consequences. Journal of thrombosis and haemostasis: JTH 2011; 9: 1713-1723

[113] Choudari CP, Rajgopal C, Palmer KR. Acute gastrointestinal haemorrhage in anticoagulated patients: diagnoses and response to endoscopic treatment. Gut 1994; 35: 464-466

[114] Rubin TA, Murdoch M, Nelson DB. Acute Gl bleeding in the setting of supratherapeutic international normalized ratio in patients taking warfarin: endoscopic diagnosis, clinical management, and outcomes. Gastrointestinal endoscopy 2003; 58: 369-373

[115] Jairath V, Kahan BC, Logan RF et al. National audit of the use of surgery and radiological embolization after failed endoscopic haemostasis for non-variceal upper gastrointestinal bleeding. The British journal of surgery 2012; 99: 1672-1680

[116] Lordkipanidze M. Is platelet transfusion the solution to reverse platelet inhibition in patients on triple antiplatelet therapy? Thrombosis research 2015; 136: $1057-1058$

[117] Bhal V, Herr M], Dixon M et al. Platelet function recovery following exposure to triple anti-platelet inhibitors using an in vitro transfusion model. Thrombosis research 2015; 136: 1216-1223

[118] Bhatt DL, Scheiman J, Abraham NS et al. ACCF/ACG/AHA 2008 expert consensus document on reducing the gastrointestinal risks of antiplatelet therapy and NSAID use: a report of the American College of Cardiology Foundation Task Force on Clinical Expert Consensus Documents. Circulation 2008; 118: 1894-1909

[119] Acosta RD, Abraham NS, Committee ASoP et al. The management of antithrombotic agents for patients undergoing Gl endoscopy. Gastrointestinal endoscopy 2016; 83: 3-16

[120] Sung J, Lau JY, Ching JY et al. Continuation of low-dose aspirin therapy in peptic ulcer bleeding: a randomized trial. Annals of internal medicine 2010; 152: 1 - 9

[121] Biondi-Zoccai GG, Lotrionte M, Agostoni P et al. A systematic review and meta-analysis on the hazards of discontinuing or not adhering to aspirin among 50,279 patients at risk for coronary artery disease. European heart journal 2006; 27: 2667 - 2674

[122] Burger W, Chemnitius JM, Kneissl GD et al. Low-dose aspirin for secondary cardiovascular prevention - cardiovascular risks after its perioperative withdrawal versus bleeding risks with its continuation review and meta-analysis. J Intern Med 2005; 257: 399-414

[123] Witt DM, Delate T, Garcia DA et al. Risk of thromboembolism, recurrent hemorrhage, and death after warfarin therapy interruption for gastrointestinal tract bleeding. Arch Intern Med 2012; 172: 1484 1491 
[124] Qureshi W, Mittal C, Patsias I et al. Restarting anticoagulation and outcomes after major gastrointestinal bleeding in atrial fibrillation. Am J Cardiol 2014; 113: $662-668$

[125] Sengupta N, Feuerstein JD, Patwardhan VR et al. The risks of thromboembolism vs. recurrent gastrointestinal bleeding after interruption of systemic anticoagulation in hospitalized inpatients with gastrointestinal bleeding: a prospective study. The American journal of gastroenterology 2015; 110: $328-335$

[126] Radaelli F, Paggi S, Terruzzi V et al. Management of warfarin-associated coagulopathy in patients with acute gastrointestinal bleeding: a cross-sectional physician survey of current practice. Digestive and liver disease: official journal of the Italian Society of Gastroenterology and the Italian Association for the Study of the Liver 2011; 43: 444-447

[127] Roudaut R, Serri K, Lafitte S. Thrombosis of prosthetic heart valves: diagnosis and therapeutic considerations. Heart 2007; 93: 137-142

[128] Maulaz AB, Bezerra DC, Michel P et al. Effect of discontinuing aspirin therapy on the risk of brain ischemic stroke. Arch Neurol 2005; 62: $1217-1220$

[129] Wolf AT, Wasan SK, Saltzman JR. Impact of anticoagulation on rebleeding following endoscopic therapy for nonvariceal upper gastrointestinal hemorrhage. The American journal of gastroenterology 2007; 102: $290-296$

[130] Skaistis J, Tagami T. Risk of Fatal Bleeding in Episodes of Major Bleeding with New Oral Anticoagulants and Vitamin K Antagonists: A Systematic Review and Meta-Analysis. PloS one 2015; 10: e0137444

[131] Sartori MT, Prandoni P. How to effectively manage the event of bleeding complications when using anticoagulants. Expert Rev Hematol 2016; 9: 37 - 50

[132] Marlu R, Hodaj E, Paris A et al. Effect of non-specific reversal agents on anticoagulant activity of dabigatran and rivaroxaban: a randomised crossover ex vivo study in healthy volunteers. Thrombosis and haemostasis 2012; 108: $217-224$

[133] Farrell J], Friedman LS. Review article: the management of lower gastrointestinal bleeding. Alimentary pharmacology \& therapeutics 2005; 21: $1281-1298$

[134] Laine L, Shah A. Randomized trial of urgent vs. elective colonoscopy in patients hospitalized with lower Gl bleeding. The American journal of gastroenterology 2010; 105: 2636-2641; quiz 2642

[135] Ohyama T, Sakurai Y, Ito M et al. Analysis of urgent colonoscopy for lower gastrointestinal tract bleeding. Digestion 2000; 61: 189-192

[136] Jensen DM, Machicado GA, Jutabha R et al. Urgent colonoscopy for the diagnosis and treatment of severe diverticular hemorrhage. The New England journal of medicine 2000; 342: 78-82

[137] Mizuki A, Tatemichi M, Hatogai K et al. Timely colonoscopy leads to faster identification of bleeding diverticulum. Nihon Shokakibyo Gakkai Zasshi 2013; 110: 1927 - 1933

[138] Yamada A, Niikura R, Yoshida S et al. Endoscopic management of colonic diverticular bleeding. Dig Endosc 2015; 27: 720-725

[139] Green BT, Rockey DC, Portwood G et al. Urgent colonoscopy for evaluation and management of acute lower gastrointestinal hemorrhage: a randomized controlled trial. The American journal of gastroenterology 2005; 100: 2395-2402

[140] Niikura R, Nagata N, Aoki T et al. Predictors for identification of stigmata of recent hemorrhage on colonic diverticula in lower gastrointestinal bleeding. Journal of clinical gastroenterology 2015; 49: e24e30

[141] Angtuaco TL, Reddy SK, Drapkin S et al. The utility of urgent colonoscopy in the evaluation of acute lower gastrointestinal tract bleeding: a 2-year experience from a single center. The American journal of gastroenterology 2001; 96: 1782-1785

[142] Adamopoulos AB, Baibas NM, Efstathiou SP et al. Differentiation between patients with acute upper gastrointestinal bleeding who need early urgent upper gastrointestinal endoscopy and those who do not.
A prospective study. European journal of gastroenterology \& hepatology 2003; 15: 381 - 387

[143] Bjorkman DJ, Zaman A, Fennerty MB et al. Urgent vs. elective endoscopy for acute non-variceal upper-GI bleeding: an effectiveness study. Gastrointestinal endoscopy 2004; 60: 1 -8

[144] Lee JG, Turnipseed S, Romano PS et al. Endoscopy-based triage significantly reduces hospitalization rates and costs of treating upper GI bleeding: a randomized controlled trial. Gastrointestinal endoscopy 1999; 50: $755-761$

[145] Wysocki JD, Srivastav S, Winstead NS. A nationwide analysis of risk factors for mortality and time to endoscopy in upper gastrointestinal haemorrhage. Alimentary pharmacology \& therapeutics 2012; 36 : $30-36$

[146] Tohda G, Higashi S, Sakumoto H et al. Efficacy and safety of nurse-administered propofol sedation during emergency upper endoscopy for gastrointestinal bleeding: a prospective study. Endoscopy 2006; 38: 684- 689

[147] Park CH, Han DS, Jeong JY et al. Outcomes of Propofol Sedation During Emergency Endoscopy Performed for Upper Gastrointestinal Bleeding. Digestive diseases and sciences 2016; 61: 825-834

[148] Yoon W, Jeong YY, Shin SS et al. Acute massive gastrointestinal bleeding: detection and localization with arterial phase multi-detector row helical CT. Radiology 2006; 239: $160-167$

[149] Scheffel H, Pfammatter T, Wildi S et al. Acute gastrointestinal bleeding: detection of source and etiology with multi-detector-row CT. European radiology 2007; 17: 1555-1565

[150] Jaeckle T, Stuber G, Hoffmann MH et al. Detection and localization of acute upper and lower gastrointestinal $(\mathrm{GI})$ bleeding with arterial phase multi-detector row helical CT. European radiology 2008; 18 : $1406-1413$

[151] Frattaroli FM, Casciani E, Spoletini D et al. Prospective study comparing multi-detector row CT and endoscopy in acute gastrointestinal bleeding. World J Surg 2009; 33: 2209-2217

[152] Kuhle WG, Sheiman RG. Detection of active colonic hemorrhage with use of helical CT: findings in a swine model. Radiology 2003; 228: $743-752$

[153] Dobritz M, Engels HP, Schneider A et al. Evaluation of dual-phase multi-detector-row CT for detection of intestinal bleeding using an experimental bowel model. European radiology 2009; 19: 875-881

[154] Chua AE, Ridley LJ. Diagnostic accuracy of CT angiography in acute gastrointestinal bleeding. J Med Imaging Radiat Oncol 2008; 52: 333 338

[155] Wu LM, Xu JR, Yin Y et al. Usefulness of CT angiography in diagnosing acute gastrointestinal bleeding: a meta-analysis. World journal of gastroenterology: WJG 2010; 16: 3957-3963

[156] Garcia-Blazquez V, Vicente-Bartulos A, Olavarria-Delgado A et al. Accuracy of CT angiography in the diagnosis of acute gastrointestinal bleeding: systematic review and meta-analysis. European radiology 2013; 23: $1181-1190$

[157] Artigas JM, Marti M, Soto JA et al. Multidetector CT angiography for acute gastrointestinal bleeding: technique and findings. Radiographics 2013; 33: $1453-1470$

[158] Geffroy Y, Rodallec MH, Boulay-Coletta I et al. Multidetector CT angiography in acute gastrointestinal bleeding: why, when, and how. Radiographics 2011; 31: E35-E46

[159] Stuber T, Hoffmann MH, Stuber G et al. Pitfalls in detection of acute gastrointestinal bleeding with multi-detector row helical CT. Abdom Imaging 2009; 34: 476-482

[160] Kennedy DW, Laing C], Tseng LH et al. Detection of active gastrointestinal hemorrhage with CT angiography: a 4(1/2)-year retrospective review. Journal of vascular and interventional radiology: JVIR 2010; 21: $848-855$ 
[161] Fisher L, Lee Krinsky M, Committee ASoP et al. The role of endoscopy in the management of obscure Gl bleeding. Gastrointestinal endoscopy 2010; 72: 471-479

[162] Bresci G, Parisi G, Bertoni M et al. The role of video capsule endoscopy for evaluating obscure gastrointestinal bleeding: usefulness of early use. Journal of gastroenterology 2005; 40: 256-259

[163] Singh A, Marshall C, Chaudhuri B et al. Timing of video capsule endoscopy relative to overt obscure Gl bleeding: implications from a retrospective study. Gastrointestinal endoscopy 2013; 77: 761-766

[164] Gilbert D, O'Malley S, Selby W. Are repeat upper gastrointestinal endoscopy and colonoscopy necessary within six months of capsule endoscopy in patients with obscure gastrointestinal bleeding? Journal of gastroenterology and hepatology 2008; 23: 1806-1809

[165] Leaper M, Johnston M], Barclay M et al. Reasons for failure to diagnose colorectal carcinoma at colonoscopy. Endoscopy 2004; 36: 499- 503

[166] Pennazio M, Spada C, Eliakim R et al. Small-bowel capsule endoscopy and device-assisted enteroscopy for diagnosis and treatment of smallbowel disorders: European Society of Gastrointestinal Endoscopy (ESGE) Clinical Guideline. Endoscopy 2015; 47: 352-376

[167] Berkelhammer C, Radvany A, Lin A et al. Heparin provocation for endoscopic localization of recurrent obscure GI hemorrhage. Gastrointestinal endoscopy 2000; 52: 555-556

[168] Raines DL, Jex KT, Nicaud MJ et al. Pharmacologic provocation combined with endoscopy in refractory cases of $\mathrm{Gl}$ bleeding. Gastrointestinal endoscopy 2017; 85: $112-120$

[169] de Leusse A, Vahedi K, Edery J et al. Capsule endoscopy or push enteroscopy for first-line exploration of obscure gastrointestinal bleeding? Gastroenterology 2007; 132: 855 - 862; quiz 1164-1165

[170] Lepileur L, Dray X, Antonietti M et al. Factors associated with diagnosis of obscure gastrointestinal bleeding by video capsule enteroscopy. Clinical gastroenterology and hepatology: the official clinical practice journal of the American Gastroenterological Association 2012; 10: $1376-1380$

[171] Bandorski D, Irnich W, Bruck M et al. Do endoscopy capsules interfere with implantable cardioverter-defibrillators? Endoscopy 2009; 41: $457-461$

[172] Herrerias JM, Leighton JA, Costamagna G et al. Agile patency system eliminates risk of capsule retention in patients with known intestinal strictures who undergo capsule endoscopy. Gastrointestinal endoscopy 2008; 67: $902-909$

[173] May A, Albert J, Keuchel M et al. Capsule endoscopy for the diagnosis of small bowel diseases. An updated statement by the endoscopy section of DGVS. Zeitschrift fur Gastroenterologie 2010; 48: 1384 1404

[174] Spada C, Riccioni ME, Costamagna G. The new, dissolving patency capsule: a safe and effective tool to avoid the complication of retained video capsules. Journal of clinical gastroenterology 2008; 42: 761 762

[175] Macdonald J, Porter V, McNamara D. Negative capsule endoscopy in patients with obscure Gl bleeding predicts low rebleeding rates. Gastrointestinal endoscopy 2008; 68: 1122-1127

[176] Min YW, Kim JS, Jeon SW et al. Long-term outcome of capsule endoscopy in obscure gastrointestinal bleeding: a nationwide analysis. Endoscopy 2014; 46: 59-65

[177] Tanizawa Y, Bando E, Kawamura T et al. Early postoperative anastomotic hemorrhage after gastrectomy for gastric cancer. Gastric Cancer 2010; 13: 50 - 57

[178] Abell TL, Minocha A. Gastrointestinal complications of bariatric surgery: diagnosis and therapy. Am J Med Sci 2006; 331: 214-218

[179] Malik AH, East JE, Buchanan GN et al. Endoscopic haemostasis of staple-line haemorrhage following colorectal resection. Colorectal disease: the official journal of the Association of Coloproctology of Great Britain and Ireland 2008; 10: 616-618
[180] Martinez-Serrano MA, Pares D, Pera M et al. Management of lower gastrointestinal bleeding after colorectal resection and stapled anastomosis. Tech Coloproctol 2009; 13: $49-53$

[181] Cirocco WC, Golub RW. Endoscopic treatment of postoperative hemorrhage from a stapled colorectal anastomosis. Am Surg 1995; 61: $460-463$

[182] Linn TY, Moran BJ, Cecil TD. Staple line haemorrhage following laparoscopic left-sided colorectal resections may be more common when the inferior mesenteric artery is preserved. Tech Coloproctol 2008; 12 : $289-293$

[183] Beyer L, Bonmardion R, Marciano S et al. Results of non-operative therapy for delayed hemorrhage after pancreaticoduodenectomy. Journal of gastrointestinal surgery: official journal of the Society for Surgery of the Alimentary Tract 2009; 13: $922-928$

[184] Yekebas EF, Wolfram L, Cataldegirmen G et al. Postpancreatectomy hemorrhage: diagnosis and treatment: an analysis in 1669 consecutive pancreatic resections. Annals of surgery 2007; 246: 269-280

[185] Vernadakis S, Christodoulou E, Treckmann J et al. Pseudoaneurysmal rupture of the common hepatic artery into the biliodigestive anastomosis. A rare cause of gastrointestinal bleeding. JOP 2009; 10: 441 444

[186] Andersson B, Nilsson J, Brandt J et al. Gastrointestinal complications after cardiac surgery. The British journal of surgery 2005; 92: $326-$ 333

[187] Sakorafas GH, Tsiotos GG. Intra-abdominal complications after cardiac surgery. Eur J Surg 1999; 165: 820-827

[188] Valentine RJ, Hagino RT, Jackson MR et al. Gastrointestinal complications after aortic surgery. Journal of vascular surgery 1998; 28: 404 411; discussion 411-402

[189] Baril DT, Carroccio A, Ellozy SH et al. Evolving strategies for the treatment of aortoenteric fistulas. Journal of vascular surgery 2006; 44 : $250-257$

[190] Peck J, Eidemiller LR. Aortoenteric fistulas. Arch Surg 1992; 127: 1191 - 1193; discussion 1193-1194

[191] Hiramoto JS, Terdiman JP, Norton JA. Evidence-based analysis: postoperative gastric bleeding: etiology and prevention. Surg Oncol 2003; 12: $9-19$

[192] Cook DJ, Fuller HD, Guyatt GH et al. Risk factors for gastrointestinal bleeding in critically ill patients. Canadian Critical Care Trials Group. The New England journal of medicine 1994; 330: 377-381

[193] Forrest JA, Finlayson ND, Shearman DJ. Endoscopy in gastrointestinal bleeding. Lancet 1974; 2: 394-397

[194] de Groot NL, van Oijen MG, Kessels K et al. Reassessment of the predictive value of the Forrest classification for peptic ulcer rebleeding and mortality: can classification be simplified? Endoscopy 2014; 46: $46-52$

[195] Elmunzer B], Young SD, Inadomi JM et al. Systematic review of the predictors of recurrent hemorrhage after endoscopic hemostatic therapy for bleeding peptic ulcers. The American journal of gastroenterology 2008; 103: 2625-2632; quiz 2633

[196] Bour B, Person B, Cales P et al. Interobserver agreement on endoscopic diagnosis of bleeding peptic ulcers. Gastrointestinal endoscopy 1997; 46: $27-32$

[197] Lau JY, Sung J], Chan AC et al. Stigmata of hemorrhage in bleeding peptic ulcers: an interobserver agreement study among international experts. Gastrointestinal endoscopy 1997; 46: 33-36

[198] Mondardini A, Barletti C, Rocca G et al. Non-variceal upper gastrointestinal bleeding and Forrest's classification: diagnostic agreement between endoscopists from the same area. Endoscopy 1998; 30: $508-512$

[199] Laine L, McQuaid KR. Endoscopic therapy for bleeding ulcers: an evidence-based approach based on meta-analyses of randomized con- 
trolled trials. Clinical gastroenterology and hepatology: the official clinical practice journal of the American Gastroenterological Association 2009; 7: 33-47

[200] Lin H], Wang K, Perng CL et al. Natural history of bleeding peptic ulcers with a tightly adherent blood clot: a prospective observation. Gastrointestinal endoscopy 1996; 43: 470-473

[201] Bleau BL, Gostout C], Sherman KE et al. Recurrent bleeding from peptic ulcer associated with adherent clot: a randomized study comparing endoscopic treatment with medical therapy. Gastrointestinal endoscopy 2002; 56: 1-6

[202] Jensen DM, Kovacs TO, Jutabha R et al. Randomized trial of medical or endoscopic therapy to prevent recurrent ulcer hemorrhage in patients with adherent clots. Gastroenterology 2002; 123: 407-413

[203] Kahi C], Jensen DM, Sung J] et al. Endoscopic therapy versus medical therapy for bleeding peptic ulcer with adherent clot: a meta-analysis. Gastroenterology 2005; 129: 855-862

[204] Petersen B, Barkun A, Carpenter S et al. Tissue adhesives and fibrin glues. Gastrointestinal endoscopy 2004; 60: 327-333

[205] Kato M, Jung Y, Gromski MA et al. Prospective, randomized comparison of 3 different hemoclips for the treatment of acute upper $\mathrm{Gl}$ hemorrhage in an established experimental setting. Gastrointestinal endoscopy 2012; 75: 3-10

[206] Chan SM, Chiu PW, Teoh AY et al. Use of the Over-The-Scope Clip for treatment of refractory upper gastrointestinal bleeding: a case series. Endoscopy 2014; 46: 428-431

[207] Manno M, Mangiafico S, Caruso A et al. First-line endoscopic treatment with OTSC in patients with high-risk non-variceal upper gastrointestinal bleeding: preliminary experience in 40 cases. Surg Endosc 2016; 30: $2026-2029$

[208] Skinner M, Gutierrez JP, Neumann H et al. Over-the-scope clip placement is effective rescue therapy for severe acute upper gastrointestinal bleeding. Endosc Int Open 2014; 2: E37-E40

[209] Barkun AN, Martel M, Toubouti Y et al. Endoscopic hemostasis in peptic ulcer bleeding for patients with high-risk lesions: a series of metaanalyses. Gastrointestinal endoscopy 2009; 69: 786 - 799

[210] Marmo R, Rotondano G, Piscopo R et al. Dual therapy versus monotherapy in the endoscopic treatment of high-risk bleeding ulcers: a meta-analysis of controlled trials. The American journal of gastroenterology 2007; 102: 279-289; quiz 469

[211] Sung J], Tsoi KK, Lai LH et al. Endoscopic clipping versus injection and thermo-coagulation in the treatment of non-variceal upper gastrointestinal bleeding: a meta-analysis. Gut 2007; 56: 1364-1373

[212] Vergara M, Bennett C, Calvet $X$ et al. Epinephrine injection versus epinephrine injection and a second endoscopic method in high-risk bleeding ulcers. The Cochrane database of systematic reviews 2014 . doi:10.1002/14651858.CD005584.pub3:CD005584

[213] Holster IL, van Beusekom HM, Kuipers EJ et al. Effects of a hemostatic powder hemospray on coagulation and clot formation. Endoscopy 2015; 47: 638-645

[214] Masci E, Arena M, Morandi E et al. Upper gastrointestinal active bleeding ulcers: review of literature on the results of endoscopic techniques and our experience with Hemospray. Scandinavian journal of gastroenterology 2014; 49: 1290-1295

[215] Smith LA, Stanley AJ, Bergman J] et al. Hemospray application in nonvariceal upper gastrointestinal bleeding: results of the Survey to Evaluate the Application of Hemospray in the Luminal Tract. Journal of clinical gastroenterology 2014; 48: e89-e92

[216] Chen YI, Barkun A, Nolan S. Hemostatic powder TC-325 in the management of upper and lower gastrointestinal bleeding: a two-year experience at a single institution. Endoscopy 2015; 47: 167-171

[217] Beg S, Al-Bakir I, Bhuva M et al. Early clinical experience of the safety and efficacy of EndoClot in the management of non-variceal upper gastrointestinal bleeding. Endosc Int Open 2015; 3: E605-E609
[218] Prei JC, Barmeyer C, Burgel N et al. EndoClot Polysaccharide Hemostatic System in Nonvariceal Gastrointestinal Bleeding: Results of a Prospective Multicenter Observational Pilot Study. Journal of clinical gastroenterology 2016; 50: e95-e100

[219] Ferlitsch A, Puspok A, Bota S et al. Efficacy and safety of bovine activated factors Ila/VIIa/IXa/Xa in patients with active gastrointestinal bleeding: a proof of concept study. Endoscopy 2016; 48: 380-384

[220] Yoshida M, Goto N, Kawaguchi M et al. Initial clinical trial of a novel hemostat, TDM-621, in the endoscopic treatments of the gastric tumors. Journal of gastroenterology and hepatology 2014; 29 (Suppl. 4): $77-79$

[221] Huang SP, Wang HP, Lee YC et al. Endoscopic hemoclip placement and epinephrine injection for Mallory-Weiss syndrome with active bleeding. Gastrointestinal endoscopy 2002; 55: 842- 846

[222] Llach J, Elizalde Jl, Guevara MC et al. Endoscopic injection therapy in bleeding Mallory-Weiss syndrome: a randomized controlled trial. Gastrointestinal endoscopy 2001; 54: 679-681

[223] Yamaguchi Y, Yamato T, Katsumi N et al. Endoscopic hemoclipping for upper $\mathrm{Gl}$ bleeding due to Mallory-Weiss syndrome. Gastrointestinal endoscopy 2001; 53: 427-430

[224] Cho YS, Chae HS, Kim HK et al. Endoscopic band ligation and endoscopic hemoclip placement for patients with Mallory-Weiss syndrome and active bleeding. World journal of gastroenterology: WJG 2008; 14: 2080-2084

[225] Swanson E, Mahgoub A, MacDonald R et al. Medical and endoscopic therapies for angiodysplasia and gastric antral vascular ectasia: a systematic review. Clinical gastroenterology and hepatology: the official clinical practice journal of the American Gastroenterological Association 2014; 12: 571 - 582

[226] Dray X, Repici A, Gonzalez P et al. Radiofrequency ablation for the treatment of gastric antral vascular ectasia. Endoscopy 2014; 46: 963 969

[227] Gross SA, Al-Haddad M, Gill KR et al. Endoscopic mucosal ablation for the treatment of gastric antral vascular ectasia with the HALO90 system: a pilot study. Gastrointestinal endoscopy 2008; 67: 324-327

[228] Jana T, Thosani N, Fallon MB et al. Radiofrequency ablation for treatment of refractory gastric antral vascular ectasia (with video). Endosc Int Open 2015; 3: E125-E127

[229] Park CH, Sohn YH, Lee WS et al. The usefulness of endoscopic hemoclipping for bleeding Dieulafoy lesions. Endoscopy 2003; 35: 388- 392

[230] Gomez V, Kyanam Kabir Baig KR, Lukens Fj et al. Novel treatment of a gastric Dieulafoy lesion with an over-the-scope clip. Endoscopy 2013; 45 (Suppl. 2): E71

[231] Alis H, Oner OZ, Kalayci MU et al. Is endoscopic band ligation superior to injection therapy for Dieulafoy lesion? Surg Endosc 2009; 23: $1465-1469$

[232] Karamanolis G, Triantafyllou K, Tsiamoulos Z et al. Argon plasma coagulation has a long-lasting therapeutic effect in patients with chronic radiation proctitis. Endoscopy 2009; 41: 529-531

[233] Alvaro-Villegas JC, Sobrino-Cossio S, Tenorio-Tellez LC et al. Argon plasma coagulation and hyperbaric oxygen therapy in chronic radiation proctopathy, effectiveness and impact on tissue toxicity. Rev Esp Enferm Dig 2011; 103: 576-581

[234] Lenz L, Tafarel J, Correia L et al. Comparative study of bipolar eletrocoagulation versus argon plasma coagulation for rectal bleeding due to chronic radiation coloproctopathy. Endoscopy 2011; 43: 697-701

[235] Akhtar K, Byrne JP, Bancewicz J et al. Argon beam plasma coagulation in the management of cancers of the esophagus and stomach. Surg Endosc 2000; 14: $1127-1130$

[236] Johnston JH, Fleischer D, Petrini J et al. Palliative bipolar electrocoagulation therapy of obstructing esophageal cancer. Gastrointestinal endoscopy 1987; 33: 349-353 
[237] Mambrini P, Audibert P, Barthet M et al. Palliative treatment of esophageal neoplastic stenosis using bipolar electrocoagulation probe. Gastroenterologie clinique et biologique 1996; 20: 844-851

[238] Kofoed SC, Lundsgaard M, Ellemann AC et al. Low morbidity after palliation of obstructing gastro-oesophageal adenocarcinoma to restore swallowing function. Dan Med J 2012; 59: A4434

[239] Kok KY, Kum CK, Goh PM. Colonoscopic evaluation of severe hematochezia in an Oriental population. Endoscopy 1998; 30: 675-680

[240] Poncet G, Heluwaert F, Voirin D et al. Natural history of acute colonic diverticular bleeding: a prospective study in 133 consecutive patients. Alimentary pharmacology \& therapeutics 2010; 32: 466-471

[241] Kaltenbach T, Watson R, Shah J et al. Colonoscopy with clipping is useful in the diagnosis and treatment of diverticular bleeding. Clinical gastroenterology and hepatology: the official clinical practice journal of the American Gastroenterological Association 2012; 10: 131 - 137

[242] Ishii N, Setoyama T, Deshpande GA et al. Endoscopic band ligation for colonic diverticular hemorrhage. Gastrointestinal endoscopy 2012; 75: $382-387$

[243] Oda I, Suzuki H, Nonaka S et al. Complications of gastric endoscopic submucosal dissection. Dig Endosc 2013; 25 (Suppl. 1): 71 - 78

[244] Fahrtash-Bahin F, Holt BA, Jayasekeran V et al. Snare tip soft coagulation achieves effective and safe endoscopic hemostasis during widefield endoscopic resection of large colonic lesions (with videos). Gastrointestinal endoscopy 2013; 78: 158 - 163 e151

[245] Burgess NG, Metz AJ, Williams S] et al. Risk factors for intraprocedural and clinically significant delayed bleeding after wide-field endoscopic mucosal resection of large colonic lesions. Clinical gastroenterology and hepatology: the official clinical practice journal of the American Gastroenterological Association 2014; 12: 651-661 e651-653

[246] Curcio G, Granata A, Traina M. Hemospray for multifocal bleeding following ultra-low rectal endoscopic submucosal dissection. Dig Endosc 2014; 26: 606-607

[247] Tsuji Y, Fujishiro M, Kodashima S et al. Polyglycolic acid sheets and fibrin glue decrease the risk of bleeding after endoscopic submucosal dissection of gastric neoplasms (with video). Gastrointestinal endoscopy 2015; 81: $906-912$

[248] Alcaide N, Penas-Herrero I, Sancho-del-Val L et al. Ovesco system for treatment of postpolypectomy bleeding after failure of conventional treatment. Rev Esp Enferm Dig 2014; 106: 55- 58

[249] Schmidt A, Gölder S, Messmann H et al. Over-the-scope-clips versus standard endoscopic therapy in patients with recurrent peptic ulcer bleeding - a prospective randomized, multicenter trial (STING). United European Gastroenterology Journal; 2016: 2 (Supplement 1)

[250] Sulz MC, Frei R, Meyenberger C et al. Routine use of Hemospray for gastrointestinal bleeding: prospective two-center experience in Switzerland. Endoscopy 2014; 46: 619-624

[251] Smith LA, Morris AJ, Stanley AJ. The use of hemospray in portal hypertensive bleeding; a case series. Journal of hepatology 2014; 60: 457 460

[252] Strate LL, Gralnek IM. ACG Clinical Guideline: Management of Patients With Acute Lower Gastrointestinal Bleeding. The American journal of gastroenterology 2016; 111: 459-474

[253] Levy M], Wong Kee Song LM, Farnell MB et al. Endoscopic ultrasound (EUS)-guided angiotherapy of refractory gastrointestinal bleeding. The American journal of gastroenterology 2008; 103: 352 - 359

[254] Holster IL, Kuipers EJ. Management of acute nonvariceal upper gastrointestinal bleeding: current policies and future perspectives. World journal of gastroenterology: WJG 2012; 18: 1202-1207

[255] Sbrozzi-Vanni A, Zullo A, Di Giulio E et al. Low prevalence of idiopathic peptic ulcer disease: an Italian endoscopic survey. Digestive and liver disease: official journal of the Italian Society of Gastroenterology and the Italian Association for the Study of the Liver 2010; 42: 773 - 776
[256] Gisbert JP, Abraira V. Accuracy of Helicobacter pylori diagnostic tests in patients with bleeding peptic ulcer: a systematic review and metaanalysis. The American journal of gastroenterology 2006; 101: 848 863

[257] Sanchez-Delgado ], Gene E, Suarez D et al. Has H. pylori prevalence in bleeding peptic ulcer been underestimated? A meta-regression. The American journal of gastroenterology 2011; 106: 398-405

[258] Kyaw M, Tse Y, Ang D et al. Embolization versus surgery for peptic ulcer bleeding after failed endoscopic hemostasis: a meta-analysis. Endosc Int Open 2014; 2: E6-E14

[259] Beggs AD, Dilworth MP, Powell SL et al. A systematic review of transarterial embolization versus emergency surgery in treatment of major nonvariceal upper gastrointestinal bleeding. Clin Exp Gastroenterol 2014; 7: $93-104$

[260] Khanna A, Ognibene S], Koniaris LG. Embolization as first-line therapy for diverticulosis-related massive lower gastrointestinal bleeding: evidence from a meta-analysis. Journal of gastrointestinal surgery: official journal of the Society for Surgery of the Alimentary Tract 2005; 9: $343-352$

[261] Padia SA, Geisinger MA, Newman JS et al. Effectiveness of coil embolization in angiographically detectable versus non-detectable sources of upper gastrointestinal hemorrhage. Journal of vascular and interventional radiology: JVIR 2009; 20: 461 -466

[262] Nanavati SM. What if endoscopic hemostasis fails? Alternative treatment strategies: interventional radiology. Gastroenterology clinics of North America 2014; 43: 739-752

[263] Farner R, Lichliter W, Kuhn J et al. Total colectomy versus limited colonic resection for acute lower gastrointestinal bleeding. American journal of surgery 1999; 178: 587-591

[264] Chiu PW, Lau JY. What if endoscopic hemostasis fails?: Alternative treatment strategies: surgery. Gastroenterology clinics of North America 2014; 43: 753-763

[265] Ripoll C, Banares R, Beceiro I et al. Comparison of transcatheter arteria embolization and surgery for treatment of bleeding peptic ulcer after endoscopic treatment failure. Journal of vascular and interventional radiology: JVIR 2004; 15: 447 - 450

[266] Wong TC, Wong KT, Chiu PW et al. A comparison of angiographic embolization with surgery after failed endoscopic hemostasis to bleeding peptic ulcers. Gastrointestinal endoscopy 2011; 73: 900 -908

[267] Morris DL, Hawker PC, Brearley S et al. Optimal timing of operation for bleeding peptic ulcer: prospective randomised trial. Br Med J (Clin Res Ed) $1984 ; 288: 1277-1280$

[268] de Franchis R, Faculty BV. Expanding consensus in portal hypertension: Report of the Baveno VI Consensus Workshop: Stratifying risk and individualizing care for portal hypertension. Journal of hepatology 2015; 63: $743-752$

[269] D’Amico G, Pasta L, Morabito A et al. Competing risks and prognostic stages of cirrhosis: a 25-year inception cohort study of 494 patients. Alimentary Pharmacology \& Therapeutics 2014; 39: 1180 - 1193

[270] Dhiman RK, Saraswat VA, Choudhuri G et al. Endosonographic, endoscopic, and histologic evaluation of alterations in the rectal venous system in patients with portal hypertension. Gastrointestinal endoscopy 1999; 49: $218-227$

[271] Ghoshal UC, Biswas PK, Roy G et al. Colonic mucosal changes in porta hypertension. Tropical gastroenterology: official journal of the Digestive Diseases Foundation 2001; 22: 25 - 27

[272] Chen LS, Lin HC, Lee FY et al. Portal hypertensive colopathy in patients with cirrhosis. Scandinavian journal of gastroenterology 1996; 31 : $490-494$

[273] Almadi MA, Almessabi A, Wong P et al. Ectopic varices. Gastrointestinal endoscopy 2011; 74: $380-388$ 
[274] Fontana RJ, Sanyal AJ, Mehta S et al. Portal hypertensive gastropathy in chronic hepatitis $C$ patients with bridging fibrosis and compensated cirrhosis: results from the HALT-C trial. The American journal of gastroenterology 2006; 101: 983-992

[275] Maurice JB, Brodkin E, Arnold F et al. Validation of the Baveno VI criteria to identify low risk cirrhotic patients not requiring endoscopic surveillance for varices. Journal of hepatology 2016; 65: 899-905

[276] Abraldes JG, Bureau C, Stefanescu $\mathrm{H}$ et al. Noninvasive tools and risk of clinically significant portal hypertension and varices in compensated cirrhosis: The "Anticipate" study. Hepatology 2016; 64: 2173-2184

[277] Garcia-Tsao G, Sanyal AJ, Grace ND et al. Prevention and management of gastroesophageal varices and variceal hemorrhage in cirrhosis; 2007: $922-938$

[278] Groszmann RJ, Garcia-Tsao G, Bosch J et al. Beta-blockers to prevent gastroesophageal varices in patients with cirrhosis. New England Journal of Medicine 2005; 353: 2254-2261

[279] Qi XS, Bao YX, Bai M et al. Nonselective beta-blockers in cirrhotic patients with no or small varices: A meta-analysis. World Journal of Gastroenterology 2015; 21:3100-3108

[280] Bhardwaj A, Kedarisetty CK, Vashishtha C et al. Carvedilol delays the progression of small oesophageal varices in patients with cirrhosis: a randomised placebo-controlled trial. Gut 2016. doi:10.1136/gutjnl2016-311735: gutjnl-2016-311735

[281] Mandorfer M, Peck-Radosavljevic M, Reiberger T. Prevention of progression from small to large varices: are we there yet? An updated meta-analysis. Gut 2016. doi:10.1136/gutjnl-2016-312814: gutjnl2016-312814-312813

[282] de Franchis R, Faculty ObotBV. Revising consensus in portal hypertension: Report of the Baveno $V$ consensus workshop on methodology of diagnosis and therapy in portal hypertension. Journal of hepatology 2010; 53: 762-768

[283] Gluud LL, Krag A. Banding ligation versus beta-blockers for primary prevention in oesophageal varices in adults. The Cochrane database of systematic reviews 2012; 8: doi:CD004544

[284] Hayes PC, Davis JM, Lewis JA et al. Meta-analysis of value of propranolol in prevention of variceal haemorrhage. The Lancet 1990; 336: 153 156

[285] Tripathi D, Ferguson JW, Kochar $\mathrm{N}$ et al. Randomized controlled trial of carvedilol versus variceal band ligation for the prevention of the first variceal bleed. Hepatology 2009; 50: 825-833

[286] Cheng JW, Zhu L, Gu MJ et al. Meta analysis of propranolol effects on gastrointestinal hemorrhage in cirrhotic patients. World Journal of Gastroenterology 2003; 9: 1836-1839

[287] Banares R, Moitinho E, Matilla A et al. Randomized comparison of longterm carvedilol and propranolol administration in the treatment of portal hypertension in cirrhosis. Hepatology 2002; 36: 1367-1373

[288] Sinagra E, Perricone G, D'Amico M et al. Systematic review with metaanalysis: the haemodynamic effects of carvedilol compared with propranolol for portal hypertension in cirrhosis. Alimentary Pharmacology Therapeutics 2014; 39: 557-568

[289] Reiberger T, Ulbrich G, Ferlitsch A et al. Carvedilol for primary prophylaxis of variceal bleeding in cirrhotic patients with haemodynamic nonresponse to propranolol. Gut 2013; 62: $1634-1641$

[290] Serste T, Melot C, Francoz C et al. Deleterious effects of beta-blockers on survival in patients with cirrhosis and refractory ascites. Hepatology 2010; 52: 1017-1022

[291] Leithead JA, Rajoriya N, Tehami N et al. Non-selective $\beta$-blockers are associated with improved survival in patients with ascites listed for liver transplantation. Gut 2015; 64: 1111-1119

[292] Mookerjee RP, Pavesi M, Thomsen KL et al. Treatment with non-selective beta blockers is associated with reduced severity of systemic inflammation and improved survival of patients with acute-on-chronic liver failure. Journal of hepatology 2016; 64: 574-582
[293] Villanueva C, Aracil C, Colomo A et al. Acute hemodynamic response to beta-blockers and prediction of long-term outcome in primary prophylaxis of variceal bleeding. Gastroenterology 2009; 137: 119-128

[294] Schepke M, Kleber G, Nürnberg D et al. Ligation versus propranolol for the primary prophylaxis of variceal bleeding in cirrhosis. Hepatology 2004; 40: $65-72$

[295] Imperiale TF, Chalasani N. A meta-analysis of endoscopic variceal ligation for primary prophylaxis of esophageal variceal bleeding. Hepatology 2001; 33: 802-807

[296] Shah HA, Azam Z, Rauf ] et al. Carvedilol vs. esophageal variceal band ligation in the primary prophylaxis of variceal hemorrhage: a multicentre randomized controlled trial. Journal of hepatology 2014; 60 : $757-764$

[297] Lo GH, Chen WC, Wang HM et al. Controlled trial of ligation plus nadolol versus nadolol alone for the prevention of first variceal bleeding. Hepatology 2010; 52: 230-237

[298] Tripathi D, Macnicholas R, Kothari C et al. Good clinical outcomes following transjugular intrahepatic portosystemic stent-shunts in BuddChiari syndrome. Alimentary Pharmacology \& Therapeutics 2014; 39 : $864-872$

[299] Thanapirom K, Ridtitid W, Rerknimitr R et al. Prospective comparison of three risk scoring systems in non-variceal and variceal upper gastrointestinal bleeding. Journal of gastroenterology and hepatology 2016; 31: $761-767$

[300] Villanueva C, Colomo A, Bosch A et al. Transfusion strategies for acute upper gastrointestinal bleeding. New England Journal of Medicine 2013; 368: $11-21$

[301] Habib A, Sanyal A]. Acute variceal hemorrhage. Gastrointestinal endoscopy clinics of North America 2007; 17: 223-252- v

[302] Kleber G, Sauerbruch T, Ansari H et al. Prediction of variceal hemorrhage in cirrhosis: a prospective follow-up study. Gastroenterology 1991; 100: 1332 - 1337

[303] Zoli M, Merkel C, Magalotti D et al. Evaluation of a new endoscopic index to predict first bleeding from the upper gastrointestinal tract in patients with cirrhosis. Hepatology 1996; 24: 1047-1052

[304] Bosch J, Abraldes JG, Berzigotti A et al. The clinical use of HVPG measurements in chronic liver disease. Nature reviews Gastroenterology \& hepatology 2009; 6: 573-582

[305] Garcia-Pagan JC, Caca K, Bureau C et al. Early use of TIPS in patients with cirrhosis and variceal bleeding. New England Journal of Medicine 2010; 362: 2370-2379

[306] Garcia-Pagan JC, Di Pascoli M, Caca K et al. Use of early-TIPS for highrisk variceal bleeding: results of a post-RCT surveillance study. Journal of hepatology 2013; 58: $45-50$

[307] Bruha R, Marecek Z, Spicak J et al. Double-blind randomized, comparative multicenter study of the effect of terlipressin in the treatment of acute esophageal variceal and/or hypertensive gastropathy bleeding. Hepato-gastroenterology 2002; 49: 1161-1166

[308] Zhou Y, Qiao L, Wu J et al. Comparison of the efficacy of octreotide, vasopressin, and omeprazole in the control of acute bleeding in patients with portal hypertensive gastropathy: a controlled study. Journal of gastroenterology and hepatology 2002; 17: 973-979

[309] Kamath PS, Lacerda M, Ahlquist DA et al. Gastric mucosal responses to intrahepatic portosystemic shunting in patients with cirrhosis. Gastroenterology 2000; 118: 905-911

[310] Escorsell A, Pavel O, Cardenas A et al. Esophageal balloon tamponade versus esophageal stent in controlling acute refractory variceal bleeding: A multicenter randomized, controlled trial. Hepatology 2016; 63: $1957-1967$

[311] McCarty TR, Njei B. Self-expanding metal stents for acute refractory esophageal variceal bleeding: A systematic review and meta-analysis. Digestive endoscopy: official journal of the Japan Gastroenterological Endoscopy Society 2016; 28: 539-547 
[312] Dechêne A, El Fouly AH, Bechmann LP et al. Acute management of refractory variceal bleeding in liver cirrhosis by self-expanding metal stents. Digestion 2012; 85: 185-191

[313] Ibrahim M, El-Mikkawy A, Abdalla $\mathrm{H}$ et al. Management of acute variceal bleeding using hemostatic powder. United European gastroenterology journal 2015; 3: $277-283$

[314] Qi X, Jia J, Bai M et al. Transjugular Intrahepatic Portosystemic Shunt for Acute Variceal Bleeding: A Meta-analysis. Journal of clinical gastroenterology 2015; 49: 495-505

[315] Halabi SA, Sawas T, Sadat B et al. Early TIPS versus endoscopic therapy for secondary prophylaxis after management of acute esophageal variceal bleeding in cirrhotic patients: a meta-analysis of randomized controlled trials. Journal of gastroenterology and hepatology 2016; 31 : $1519-1526$

[316] Gülberg V, Schepke M, Geigenberger G et al. Transjugular intrahepatic portosystemic shunting is not superior to endoscopic variceal band ligation for prevention of variceal rebleeding in cirrhotic patients: a randomized, controlled trial. Scandinavian journal of gastroenterology 2002; 37: $338-343$

[317] Pomier-Layrargues G, Villeneuve JP, Deschênes M et al. Transjugular intrahepatic portosystemic shunt (TIPS) versus endoscopic variceal ligation in the prevention of variceal rebleeding in patients with cirrhosis: a randomised trial. Gut 2001; 48: 390-396

[318] Sauer P, Hansmann J, Richter GM et al. Endoscopic variceal ligation plus propranolol vs. transjugular intrahepatic portosystemic stent shunt: a long-term randomized trial. Endoscopy 2002; 34: 690-697

[319] Burroughs AK, Vangeli M. Transjugular intrahepatic portosystemic shunt versus endoscopic therapy: randomized trials for secondary prophylaxis of variceal bleeding: an updated meta-analysis. Scandinavian journal of gastroenterology 2002; 37: 249-252

[320] Papatheodoridis GV, Goulis ], Leandro G et al. Transjugular intrahepatic portosystemic shunt compared with endoscopic treatment for prevention of variceal rebleeding: A meta-analysis. Hepatology 1999; 30: $612-622$

[321] Zheng M, Chen Y, Bai ] et al. Transjugular intrahepatic portosystemic shunt versus endoscopic therapy in the secondary prophylaxis of variceal rebleeding in cirrhotic patients: meta-analysis update. Journal of clinical gastroenterology 2008; 42: 507 - 516

[322] Sauerbruch T, Mengel M, Dollinger M et al. Prevention of Rebleeding From Esophageal Varices in Patients With Cirrhosis Receiving SmallDiameter Stents Versus Hemodynamically Controlled Medical Therapy. Gastroenterology 2015; 149: 660-668.e661

[323] Barrio ], Ripoll C, Bañares R et al. Comparison of transjugular intrahepatic portosystemic shunt dysfunction in PTFE-covered stent-grafts versus bare stents. European journal of radiology 2005; 55: 120-124

[324] Bureau C, Pagán JCG, Layrargues GP et al. Patency of stents covered with polytetrafluoroethylene in patients treated by transjugular intrahepatic portosystemic shunts: long-term results of a randomized multicentre study. Liver international: official journal of the International Association for the Study of the Liver 2007; 27: 742 - 747

[325] Boyer TD, Haskal Z], Diseases AAftSoL. The Role of Transjugular Intrahepatic Portosystemic Shunt (TIPS) in the Management of Portal Hypertension: update 2009. Wiley Subscription Services, Inc., A Wiley Company 2010: 306

[326] Tesdal IK, Filser T, Weiss C et al. Transjugular intrahepatic portosystemic shunts: adjunctive embolotherapy of gastroesophageal collateral vessels in the prevention of variceal rebleeding. Radiology 2005; 236: $360-367$

[327] Chen S, Li X, Wei B et al. Recurrent variceal bleeding and shunt patency: prospective randomized controlled trial of transjugular intrahepatic portosystemic shunt alone or combined with coronary vein embolization. Radiology 2013; 268: 900 -906
[328] Park JK, Saab S, Kee ST et al. Balloon-Occluded Retrograde Transvenous Obliteration (BRTO) for Treatment of Gastric Varices: Review and Meta-Analysis. Digestive diseases and sciences 2015; 60: 1543-1553

[329] Henderson JM, Boyer TD, Kutner MH et al. Distal Splenorenal Shunt Versus Transjugular Intrahepatic Portal Systematic Shunt for Variceal Bleeding: A Randomized Trial. Gastroenterology 2006; 130: 1643 1651

[330] Bernard B, Cadranel JF, Valla D et al. Prognostic significance of bacteria infection in bleeding cirrhotic patients: a prospective study. Gastroenterology 1995; 108: 1828-1834

[331] Bleichner G, Boulanger R, Squara P et al. Frequency of infections in cirrhotic patients presenting with acute gastrointestinal haemorrhage. The British journal of surgery 1986; 73: $724-726$

[332] Hou MC, Lin HC, Liu TT et al. Antibiotic prophylaxis after endoscopic therapy prevents rebleeding in acute variceal hemorrhage: a randomized trial. Hepatology 2004; 39: 746 - 753

[333] Hsieh W], Lin HC, Hwang S] et al. The effect of ciprofloxacin in the prevention of bacterial infection in patients with cirrhosis after upper gastrointestinal bleeding. The American journal of gastroenterology 1998; $93: 962-966$

[334] Soriano G, Guarner C, Tomas A et al. Norfloxacin prevents bacterial infection in cirrhotics with gastrointestinal hemorrhage. Gastroenterology 1992; 103: $1267-1272$

[335] Pauwels A, Mostefa-Kara N, Debenes B et al. Systemic antibiotic prophylaxis after gastrointestinal hemorrhage in cirrhotic patients with a high risk of infection. Hepatology 1996; 24: $802-806$

[336] Brown MR, Jones G, Nash KL et al. Antibiotic prophylaxis in variceal hemorrhage: timing, effectiveness and Clostridium difficile rates. World journal of gastroenterology: WJG 2010; 16: 5317-5323

[337] Ioannou G, Doust J, Rockey DC. Terlipressin for acute esophageal variceal hemorrhage. The Cochrane database of systematic reviews 2003. doi:10.1002/14651858.CD002147: CD002147

[338] Tripodi A, Mannucci PM. The coagulopathy of chronic liver disease. The New England journal of medicine 2011; 365: 147-156

[339] Tripodi A, Salerno F, Chantarangkul V et al. Evidence of normal thrombin generation in cirrhosis despite abnormal conventional coagulation tests. Hepatology 2005; 41: $553-558$

[340] Bosch J, Thabut D, Albillos A et al. Recombinant factor VIla for variceal bleeding in patients with advanced cirrhosis: A randomized, controlled trial. Hepatology 2008; 47: 1604-1614

[341] Bosch J, Thabut D, Bendtsen F et al. Recombinant factor VIla for upper gastrointestinal bleeding in patients with cirrhosis: a randomized, double-blind trial. Gastroenterology 2004; 127: 1123-1130

[342] Bendtsen F, D'Amico G, Rusch E et al. Effect of recombinant Factor VIla on outcome of acute variceal bleeding: an individual patient based meta-analysis of two controlled trials. Journal of hepatology 2014; 61: $252-259$

[343] Jairath V, Rehal S, Logan R et al. Acute variceal haemorrhage in the United Kingdom: patient characteristics, management and outcomes in a nationwide audit. Digestive and liver disease: official journal of the Italian Society of Gastroenterology and the Italian Association for the Study of the Liver 2014; 46: 419-426

[344] Sorbi D, Gostout C], Peura D et al. An assessment of the management of acute bleeding varices: a multicenter prospective member-based study. The American journal of gastroenterology 2003; 98: 2424 2434

[345] Lo EA, Wilby KJ, Ensom MH. Use of proton pump inhibitors in the management of gastroesophageal varices: a systematic review. Ann Pharmacother 2015; 49: 207-219

[346] Sharma P, Agrawal A, Sharma BC et al. Prophylaxis of hepatic encephalopathy in acute variceal bleed: a randomized controlled trial of lactulose versus no lactulose. Journal of gastroenterology and hepatology 2011; 26: $996-1003$ 
[347] Maharshi S, Sharma BC, Srivastava S et al. Randomised controlled trial of lactulose versus rifaximin for prophylaxis of hepatic encephalopathy in patients with acute variceal bleed. Gut 2015; 64: 1341 - 1342

[348] Bari K, Garcia-Tsao G. Treatment of portal hypertension. World journal of gastroenterology: WJG 2012; 18: 1166-1175

[349] Gatta A, Merkel C, Sacerdoti D et al. Nadolol for prevention of variceal rebleeding in cirrhosis: a controlled clinical trial. Digestion 1987; 37 : $22-28$

[350] D'Amico G, Pagliaro L, Bosch J. Pharmacological treatment of portal hypertension: an evidence-based approach. Seminars in liver disease 1999; 19: 475-505

[351] Gournay J, Masliah C, Martin T et al. Isosorbide mononitrate and propranolol compared with propranolol alone for the prevention of variceal rebleeding. Hepatology 2000; 31: 1239-1245

[352] Gluud LL, Langholz E, Krag A. Meta-analysis: isosorbide-mononitrate alone or with either beta-blockers or endoscopic therapy for the management of oesophageal varices. Alimentary pharmacology \& therapeutics 2010; 32: 859-871

[353] Lo GH, Chen WC, Wang HM et al. Randomized, controlled trial of carvedilol versus nadolol plus isosorbide mononitrate for the prevention of variceal rebleeding. Journal of gastroenterology and hepatology 2012; 27: $1681-1687$

[354] Abraldes JG, Villanueva C, Aracil C et al. Addition of Simvastatin to Standard Therapy for the Prevention of Variceal Rebleeding Does Not Reduce Rebleeding but Increases Survival in Patients With Cirrhosis. Gastroenterology 2016; 150: 1160 - 1170 e1163

[355] Lo GH, Chen WC, Chen MH et al. Banding ligation versus nadolol and isosorbide mononitrate for the prevention of esophageal variceal rebleeding. Gastroenterology 2002; 123: 728-734

[356] Lo GH, Chen WC, Lin CK et al. Improved survival in patients receiving medical therapy as compared with banding ligation for the prevention of esophageal variceal rebleeding. Hepatology 2008; 48: 580-587

[357] Li L, Yu C, Li Y. Endoscopic band ligation versus pharmacological therapy for variceal bleeding in cirrhosis: a meta-analysis. Canadian journal of gastroenterology = Journal canadien de gastroenterologie 2011; 25: $147-155$

[358] Cheung J, Zeman M, van Zanten SV et al. Systematic review: secondary prevention with band ligation, pharmacotherapy or combination therapy after bleeding from oesophageal varices. Alimentary pharmacology \& therapeutics 2009; 30: 577-588

[359] Ding SH, Liu J, Wang JP. Efficacy of beta-adrenergic blocker plus 5-isosorbide mononitrate and endoscopic band ligation for prophylaxis of esophageal variceal rebleeding: a meta-analysis. World journal of gastroenterology: WJG 2009; 15: 2151-2155

[360] Ravipati M, Katragadda S, Swaminathan PD et al. Pharmacotherapy plus endoscopic intervention is more effective than pharmacotherapy or endoscopy alone in the secondary prevention of esophageal variceal bleeding: a meta-analysis of randomized, controlled trials. Gastrointestinal endoscopy 2009; 70: 658-664 e655

[361] Stanley AJ, Dickson S, Hayes PC et al. Multicentre randomised controlled study comparing carvedilol with variceal band ligation in the prevention of variceal rebleeding. Journal of hepatology 2014; 61: $1014-1019$

[362] Thiele M, Krag A, Rohde U et al. Meta-analysis: banding ligation and medical interventions for the prevention of rebleeding from oesophageal varices. Alimentary Pharmacology \& Therapeutics 2012; 35: $1155-1165$

[363] Puente A, Hernandez-Gea V, Graupera I et al. Drugs plus ligation to prevent rebleeding in cirrhosis: an updated systematic review. Liver international: official journal of the International Association for the Study of the Liver 2014; 34: 823-833
[364] Mandorfer M, Bota S, Schwabl P et al. Nonselective beta blockers increase risk for hepatorenal syndrome and death in patients with cirrhosis and spontaneous bacterial peritonitis. Gastroenterology 2014; 146: 1680 - 1690 e1681

[365] Mishra SR, Chander Sharma B, Kumar A et al. Endoscopic cyanoacrylate injection versus beta-blocker for secondary prophylaxis of gastric variceal bleed: a randomised controlled trial. Gut 2010; 59: 729-735

[366] Luca A, D'Amico G, La Galla R et al. TIPS for prevention of recurrent bleeding in patients with cirrhosis: meta-analysis of randomized clinical trials. Radiology 1999; 212: 411-421

[367] Khan S, Tudur Smith C, Williamson P et al. Portosystemic shunts versus endoscopic therapy for variceal rebleeding in patients with cirrhosis. The Cochrane database of systematic reviews 2006. doi:10.1002/ 14651858.CD000553.pub2: CD000553

[368] Windecker S, Kolh P, Authors/Task Force m et al. 2014 ESC/EACTS Guidelines on myocardial revascularization: The Task Force on Myocardial Revascularization of the European Society of Cardiology (ESC) and the European Association for Cardio-Thoracic Surgery (EACTS)Developed with the special contribution of the European Association of Percutaneous Cardiovascular Interventions (EAPCI). European heart journal 2014; 35: 2541 - 2619

[369] Roffi M, Patrono C, Collet JP et al. 2015 ESC Guidelines for the management of acute coronary syndromes in patients presenting without persistent ST-segment elevation: Task Force for the Management of Acute Coronary Syndromes in Patients Presenting without Persistent ST-Segment Elevation of the European Society of Cardiology (ESC). European heart journal 2016; 37: 267-315

[370] Adler DG, Leighton JA, Davila RE et al. ASGE guideline: The role of endoscopy in acute non-variceal upper-GI hemorrhage. Gastrointestinal endoscopy 2004; 60: 497-504

[371] El Ouali S, Barkun AN, Wyse J et al. Is routine second-look endoscopy effective after endoscopic hemostasis in acute peptic ulcer bleeding? A meta-analysis. Gastrointestinal endoscopy 2012; 76: 283-292

[372] Chiu PW, Joeng HK, Choi CL et al. High-dose omeprazole infusion compared with scheduled second-look endoscopy for prevention of peptic ulcer rebleeding: a randomized controlled trial. Endoscopy 2016; 48: 717-722

[373] Imperiale TF, Kong N. Second-look endoscopy for bleeding peptic ulcer disease: a decision-effectiveness and cost-effectiveness analysis. Journal of clinical gastroenterology 2012; 46: e71 - e75

[374] Qureshi W, Adler DG, Davila R et al. ASGE Guideline: the role of endoscopy in the management of variceal hemorrhage, updated July 2005. Gastrointestinal endoscopy 2005; 62: 651-655

[375] Riphaus A, Wehrmann T, Weber B et al. S3-guidelines-sedation in gastrointestinal endoscopy. Zeitschrift fur Gastroenterologie 2008; 46: $1298-1330$

[376] Waring JP, Baron TH, Hirota WK et al. Guidelines for conscious sedation and monitoring during gastrointestinal endoscopy. Gastrointestinal endoscopy 2003; 58: 317-322

[377] Taeger K. Leitlinie zur Sedierung und Analgesie (Analgosedierung) von Patienten durch Nicht-Anästhesisten. Anästh Intensivmed 2002; 43: $639-641$

[378] Amornyotin S, Chal W, Kongphlay S. Recovery pattern and homereadiness after ambulatory gastrointestinal endoscopy. J Med Assoc Thai 2007; 90: 2352-2358

[379] Dumonceau JM, Riphaus A, Schreiber F et al. Non-anesthesiologist administration of propofol for gastrointestinal endoscopy: European Society of Gastrointestinal Endoscopy, European Society of Gastroenterology and Endoscopy Nurses and Associates Guideline-Updated June 2015. Endoscopy 2015; 47: 1175-1189 